\title{
Link-Layer Retransmission-based Error-Control Protocols in FSO Communications: A Survey
}

\author{
Hoang D. Le, Student Member, IEEE, and Anh T. Pham, Senior Member, IEEE
}

\begin{abstract}
Free-space optical (FSO) communications have gained significant interest over the last few years, thanks to the capability to transport extremely high-speed data over long distances without exhausting radio frequency (RF) resources. FSO communication is widely considered in various network scenarios, such as inter-satellite/deep-space links, ground-station/vehicles, satellite/aerial links, or terrestrial links. It is expected to be a key enabling technology for the next generation of $6 \mathrm{G}$ wireless networks. Nevertheless, despite the great potential of FSO communications, its performance suffers from various limitations and challenges: atmospheric turbulence, clouds, weather conditions, and pointing misalignment. The error-control solutions, including physical layer (PHY) and link-layer methods, aim to mitigate the transmission errors caused by such adverse issues. While the existing surveys on error-control solutions in FSO systems primarily focussed on the PHY methods, we instead provide a review of link-layer solutions. In particular, we conduct an extensive literature survey of state-of-the-art retransmission protocols, both automatic repeat request $(A R Q)$ and hybrid $A R Q$ (HARQ), for various FSO communication scenarios, including point-to-point terrestrial, cooperative, multi-hop relaying, hybrid FSO/RF, satellite/aerial, and deep-space systems. Furthermore, we provide a survey of recent literature and insightful discussion on the cross-layer design frameworks related to link-layer retransmission protocols in FSO communication networks. Finally, the lessons learned, design guidelines, related open issues, and future research directions are exposed.
\end{abstract}

Index Terms-Free-space optical (FSO) communications, link layer error-control protocols, automatic repeat request (ARQ), hybrid ARQ (HARQ), cross-layer design.

\section{NOMENClature}

$\begin{array}{ll}\text { 5G } & \text { Fifth Generation Wireless Mobile Network } \\ 6 G & \text { Sixth Generation Wireless Mobile Network } \\ \text { ACK } & \text { Acknowledgement } \\ \text { AI } & \text { Artificial Intelligent. } \\ \text { AF } & \text { Amplify-and-Forward } \\ \text { AMC } & \text { Adaptive Modulation and Coding } \\ \text { AoA } & \text { Angle of Arrival } \\ \text { AP } & \text { Adaptive Power } \\ \text { AR } & \text { Adaptive Rate } \\ \text { ARQ } & \text { Automatic Repeat Request } \\ \text { BER } & \text { Bit Error Rate } \\ \text { C-ARQ } & \text { Cooperative Automatic Repeat Request } \\ \text { CC } & \text { Chase Combining } \\ \text { C-HARQ } & \text { Cooperative Hybrid Automatic Repeat Request } \\ \text { TBIRD } & \text { Terabyte Infrared Delivery } \\ \text { CRC } & \text { Standard Cyclic Redundancy Check }\end{array}$

Hoang D. Le and Anh T. Pham are with the Computer Communications Lab., The University of Aizu, Aizuwakamatsu 965-8580, Japan (email: hoangbkset@gmail.com,pham@u-aizu.ac.jp).

$\begin{array}{ll}\text { CSI } & \text { Channel State Information } \\ \text { DF } & \text { Decode-and-Forward } \\ \text { DLR } & \text { German Aerospace Centre } \\ \text { DTN } & \text { Disruption-Tolerant Networking } \\ \text { ECC } & \text { Error Correction Code } \\ \text { EE } & \text { Energy Efficiency } \\ \text { FC } & \text { Frame Combining } \\ \text { FEC } & \text { Forward Error Correction } \\ \text { FER } & \text { Frame Error Rate } \\ \text { FLR } & \text { Frame Loss Rate } \\ \text { FSO } & \text { Free-Space Optical } \\ \text { GBN } & \text { Go-Back-N } \\ \text { HAPs } & \text { High Altitude Platforms } \\ \text { HARQ } & \text { Hybrid Automatic Repeat Request } \\ \text { IM/DD } & \text { Intensity Modulation/Direct Detection } \\ \text { IoVs } & \text { Internet of Vehicles } \\ \text { IP } & \text { Internet Protocol } \\ \text { IR } & \text { Incremental Redundancy } \\ \text { LEO } & \text { Low Earth Orbit } \\ \text { LFN } & \text { Long Fat Network } \\ \text { LT } & \text { Luby Transform } \\ \text { LTP } & \text { Licklider Transmission Protocol } \\ \text { M-C-ARQ } & \text { Modified Cooperative Automatic Repeat Re- } \\ & \text { quest } \\ \text { mmWave } & \text { Millimeter-Wave } \\ \text { MRC } & \text { Maximum Ratio Combining } \\ \text { mURLLC } & \text { Massive ultra-reliable and low latency commu- } \\ & \text { nications } \\ \text { NAK } & \text { Negative Acknowledgement } \\ \text { NASA } & \text { National Aeronautics and Space Administration } \\ \text { NOMA } & \text { Non-Orthogonal Multiple Access } \\ \text { OSI } & \text { Open Systems Interconnection } \\ \text { PAA } & \text { Point-Ahead-Angle } \\ \text { PHY } & \text { Physical Layer } \\ \text { QKD } & \text { Quantum Key Distribution } \\ \text { QoS } & \text { Quality of Service } \\ \text { Qubit } & \text { Quantum Bit } \\ \text { RCPC } & \text { Rate-Compatible Punctured Convolutional } \\ \text { RF } & \text { Radio Frequency } \\ \text { RS } & \text { Reed-Solomon } \\ \text { RTT } & \text { Round Trip Time } \\ \text { SACK } & \text { TCP-Selective Acknowledgement } \\ \text { SE } & \text { Spectral Efficiency } \\ \text { SI } & \text { Scintillation Index } \\ \text { SLP } & \text { Segment Loss Probability } \\ \text { SNR } & \text { Signal-to-Noise Ratio } \\ \text { SR } & \text { Selective Repeat } \\ \text { SW } & \text { Stop-and-Wait } \\ & \end{array}$




$\begin{array}{ll}\text { TCP } & \text { Transmission Control Protocol } \\ \text { TE } & \text { Transmission Efficiency } \\ \text { TI-HARQ } & \text { Type I-Hybrid Automatic Repeat Request } \\ \text { UAVs } & \text { Unmanned Aerial Vehicles } \\ \text { UWAN } & \text { Underwater Acoustic Network } \\ \text { VR } & \text { Virtual Reality }\end{array}$

\section{INTRODUCTION}

$\mathbf{T}$ HE rapid development of various emerging applications, such as artificial intelligence (AI), virtual reality (VR), and Internet of Vehicles (IoVs), has produced a massive volume of data traffic that requires high-speed wireless connectivity [1]. According to the International Telecommunication Union (ITU), the global mobile traffic volume was 7.462 $\mathrm{EB} /$ month in 2010 and is predicted to be $5016 \mathrm{~EB} / \mathrm{month}$ in 2030 [2]. Indeed, the support of this extremely high volume data poses a significant challenge for the forthcoming fifthgeneration $(5 \mathrm{G})$ and beyond wireless communication networks.

It is well established that the current communications based on radio frequency $(\mathrm{RF})$ are becoming more restricted due to the limited spectrum resources and may not satisfy this growing demand [3]-[5]. As a result, recent years have witnessed an increasing interest in free-space optical (FSO) communications research and development. FSO is being considered as a promising complementary candidate to the current RF, thanks to the enormous available unlicensed bandwidth and the capability of transmission at very high data rates over long distances [6]. Furthermore, as compared to existing RF-based wireless systems, the narrow and directional characteristics of a laser beam employed in FSO communications enable a high level of security, a low power consumption, and an immunity to electromagnetic interference [7].

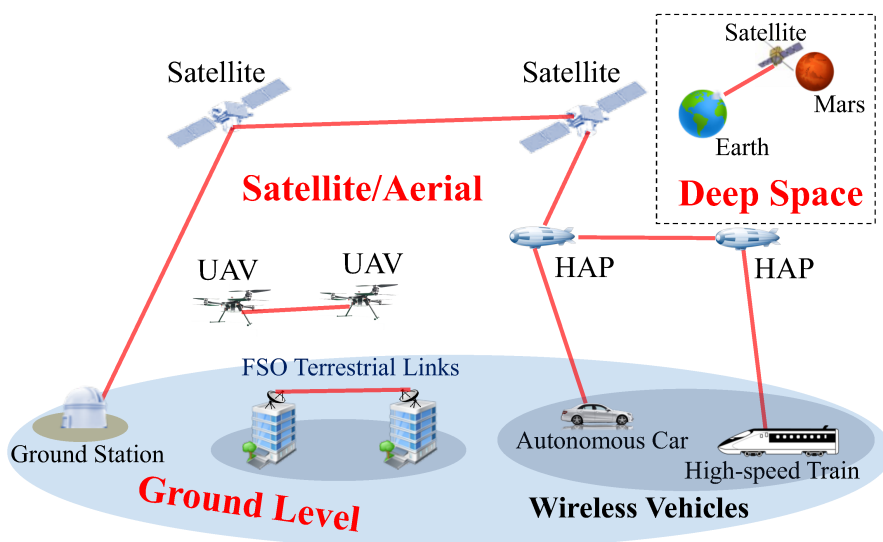

Figure 1: An example of FSO communication networks.

FSO systems can be classified into two broad categories, i.e., terrestrial and space links [8]. As an example illustrated in Fig. 1, the terrestrial links can be a connection between building-to-building. In addition, the space links include interorbital (e.g., satellite-to-vehicles, between unmanned aerial vehicles (UAVs) or high-altitude platforms (HAPs)), intersatellite, and deep space links. The FSO transmissions through the atmosphere are, nevertheless, not without challenges. The primary concerns of FSO links is briefly summarized in Table I, in which different systems may experience different adverse issues [9], [10]. Indeed, those adverse issues pose various challenges to the performance of FSO systems, which requires a lot of research efforts for tackling.

Table I: Major adverse issues on diferent FSO links.

\begin{tabular}{|l|l|}
\hline FSO Links & Main Challenging Issues \\
\hline \hline Deep Space & $\begin{array}{l}\text { Path Loss, Atmospheric Turbulence, Misalign- } \\
\text { ment Errors, Coronal Solar Wind Turbulence }\end{array}$ \\
\hline Inter-Satellite & $\begin{array}{l}\text { Pointing Errors, Doppler Shift, Point-Ahead- } \\
\text { Angle (PAA) }\end{array}$ \\
\hline Satellite-to-HAP & Pointing Errors, Geometric Loss \\
\hline HAP-to-HAP & Pointing Errors, Turbulence (weak) \\
\hline UAV-to-UAV & $\begin{array}{l}\text { Pointing Errors, Turbulence (weak-to-strong), } \\
\text { Angle-of-Arrival (AoA) Fluctuations }\end{array}$ \\
\hline Satellite-to-Ground & $\begin{array}{l}\text { Pointing Errors, Turbulence-induced Scintilla- } \\
\text { tion (weak), Clouds, Geometric Loss }\end{array}$ \\
\hline Ground-to-Satellite & $\begin{array}{l}\text { Pointing Errors, Turbulence-induced Beam } \\
\text { Wander, Clouds, Geometric Loss }\end{array}$ \\
\hline Building-to-Building & $\begin{array}{l}\text { Pointing Errors, Turbulence (weak-to-strong), } \\
\text { Fogs }\end{array}$ \\
\hline
\end{tabular}

\section{A. Error-Control Methods in FSO Communications}

Extensive studies have been devoted to error-control solutions, which can be mainly categorized into two groups: physical (PHY) layer and link-layer methods. Notably, the PHY methods widely used in FSO communications include adaptive modulation and coding (AMC) [11]-[13], adaptive rate $(\mathrm{AR}) /$ power (AP) transmissions [14], [15], forward error correction (FEC) code [16]-[19], multi-hop transmissions [20]-[22], cooperative diversity technique [23], [24], aperture averaging [25], hybrid RF/FSO [26]-[28], and signal processing techniques [29]-[31]. Such methods can considerably mitigate the transmission errors caused by the aforementioned adverse issues on FSO links, thus improving the system's reliability and availability. For instance, as reported in [17], by using FEC-based Turbo codes with a coding rate of $1 / 3$, the terrestrial FSO systems can retain a level of bit error rate (BER) of $10^{-6}$ in the moderate-to-strong turbulence conditions. They also reported that a lower coding rate is needed to maintain a lower BER level, leading to the inefficiency in system throughput performance under time-varying channel conditions, as many redundancy bits are required. As a result, it is difficult and not cost-effective for the PHY layer to guarantee error-free reception.

To further enhance the reliability and efficiency in communication systems, link-layer error-control methods, including redundancy and retransmission mechanisms, have been widely investigated in the context of FSO communications. A typical redundancy mechanism is the error correction code (ECC), which guarantees transmission reliability by adding some redundancies to the original message so that receivers can use them to recover the erroneous data [32]. In addition, ARQ is one of the well-known retransmission mechanisms, which facilitates the retransmission of erroneously received frames via feedback from the receiver to the transmitter [33], [34]. Under the impact of severe channel impairments, a more robust retransmission-based error-control method, i.e., hybrid 
Table II: Summary of existing surveys relating to error-control methods in FSO communications.

\begin{tabular}{|c|c|c|c|c|c|c|c|}
\hline \multirow{2}{*}{ Survey Paper } & \multirow{2}{*}{ Year } & \multirow{2}{*}{ Addressed Issues } & \multicolumn{3}{|c|}{ Error-Control Methods } & \multicolumn{2}{|c|}{ Networks } \\
\hline & & & $\begin{array}{l}\text { PHY } \\
\text { Layer }\end{array}$ & $\begin{array}{c}\text { Link } \\
\text { Layer }\end{array}$ & $\begin{array}{c}\text { PHY/Link } \\
\text { Cross-Layer }\end{array}$ & Terrestrial & Aerial \\
\hline Khalighi et al. [37] & 2014 & $\begin{array}{c}\text { Channel coding, spatial diversity } \\
\text { technique, adaptive transmissions, } \\
\text { relay-assisted cooperative transmissions, } \\
\text { hybird FSO/RF }\end{array}$ & $\checkmark \checkmark$ & & & $\checkmark$ & \\
\hline Kaushal et al. [38] & 2017 & $\begin{array}{l}\text { Aperture averaging, diversity, relay } \\
\text { transmissions, adaptive optics, signal } \\
\text { processing techniques, hybrid FSO/RF }\end{array}$ & $\checkmark$ & & & & $\checkmark$ \\
\hline Son et al. [39] & 2017 & $\begin{array}{l}\text { Cooperative diversity technique, } \\
\text { relay-assisted communications, hybrid } \\
\text { FSO/RF, signal processing techniques }\end{array}$ & $\checkmark$ & & & $\checkmark$ & $\checkmark$ \\
\hline Vavoulas et al. [40] & 2019 & $\begin{array}{l}\text { Signal processing techniques, diversity } \\
\text { techniques, FEC code }\end{array}$ & $\checkmark$ & & & $\checkmark$ & \\
\hline Trichili et al. [41] & 2019 & Signal processing techniques & $\checkmark$ & & & $\checkmark$ & \\
\hline Raj et al. [42] & 2019 & $\begin{array}{c}\text { Aperture averaging, waveform correction, } \\
\text { hybrid FSO/RF }\end{array}$ & $\checkmark$ & & & $\checkmark$ & $\checkmark$ \\
\hline Chen et al. [43] & 2020 & $\begin{array}{l}\text { Signal processing techniques } \\
\end{array}$ & $\checkmark$ & & & $\checkmark$ & \\
\hline Chowdhury et al. [44] & 2020 & Hybrid FSO/RF & $\checkmark$ & & & $\checkmark$ & $\checkmark$ \\
\hline Liu et al. [45] & 2020 & $\begin{array}{l}\text { Relay-assisted technology, signal } \\
\text { processing techniques, channel coding, } \\
\text { adaptive transmission, cooperative } \\
\text { diversity }\end{array}$ & $\checkmark$ & & & $\checkmark$ & $\checkmark$ \\
\hline Trichili et al. [46] & 2020 & $\begin{array}{c}\text { Channel coding, diversity, adaptive optics, } \\
\text { relay transmission, signal processing } \\
\text { techniques, hybrid FSO/RF }\end{array}$ & $\checkmark$ & & & $\checkmark$ & \\
\hline This paper & & $\begin{array}{l}\text { Link-layer retransmission solutions, both } \\
\text { ARQ and HARQ, and the cross-layer } \\
\text { design with PHY methods }\end{array}$ & & $\checkmark$ & $\checkmark$ & $\checkmark$ & $\checkmark$ \\
\hline
\end{tabular}

ARQ (HARQ), which achieves better reliability by combining ARQ and ECC, is preferable to standard ARQ and ECC in some scenarios, e.g., long-distance satellite communication systems [35], [36]. However, the main drawbacks of HARQ protocols are system complexity, additional signaling, and large overhead [33].

\section{B. Relevant Survey/Tutorial Articles}

A couple of surveys and tutorials related to error-control methods appeared in the literature of FSO communications, which mainly focused on the PHY layer solutions [37][46]. Khalighi et al. reviewed several PHY layer error-control methods used in FSO-based terrestrial systems, including FEC code, spatial diversity technique, adaptive transmissions, relay-assisted cooperative transmissions, and hybrid FSO/RF schemes in [37]. A survey paper by Kaushal et al. [38] summarized and reviewed recent works on the PHY errorcontrol solutions in FSO-based space communications, including satellite-to-ground, ground-to-satellite, and inter-satellite systems. Various PHY methods, e.g., aperture averaging, diversity, relay transmissions, adaptive optics, signal processing techniques, hybrid FSO/RF, were presented for such systems. Another survey by Son et al. [39] focused on both terrestrial and space communications, in which a short review of PHY error-control methods was provided, i.e., cooperative diversity technique, relay-assisted communications, hybrid FSO/RF, and signal processing techniques. Vavoulas et al. [40] presented a survey on ultraviolet C-band for FSO communications and PHY methods, i.e., signal processing techniques, diversity techniques, and FEC code for such systems. The surveys and tutorials on signal processing technique of PHY error- control methods were reported in [41] and [43]. In addition, Raj et al. [42] presented the state-of-art developments of FSO communications, wherein several PHY solutions, i.e., aperture averaging, waveform correction, and hybrid FSO/RF, were reviewed. In [44], Chowdhury et al. provided a comprehensive overview of existing literature on optical wireless hybrid networks, such as RF/optical and optical/optical systems. A relayassisted technology in FSO communications was surveyed by Liu et al. [45] for various systems, from the terrestrial to space communications. They also presented a literature review regarding the signal processing techniques, channel coding, adaptive transmission, cooperative diversity for FSO communications. Most recently, Trichili et al. [46] provided an up-todate review of PHY error-control solutions, containing channel coding, diversity, adaptive optics, relay transmission, signal processing techniques, and hybrid FSO/RF, in terrestrial FSO communications. The aforementioned surveys with regards to error-control methods are summarized at a glance in Table II, which allows readers to capture the major contributions of each of the existing surveys.

At the time of writing, we realize that a survey of link-layer error-control solutions is not available in the literature of FSO communications. Among link-layer error-control solutions, retransmission-based methods, including ARQ and HARQ, have more degrees of freedom as compared to ECC schemes, making them a fertile research area in the domain of FSO communications. These protocols have been widely surveyed in the literature of RF communications [47]-[55]. A summary of these works is shown in Table III. However, we have to point out that a proper design and performance evaluation of such protocols should be examined and investigated carefully 
Table III: Existing surveys/tutorials relating to link-layer retransmission protocols.

\begin{tabular}{|c|c|c|}
\hline Year & Publication & One-Sentence Summary \\
\hline 1984 & Lin et al. [47] & A review of ARQ and HARQ protocols in $R F$ communications \\
\hline 2011 & Cola et al. [48] & A survey on ARQ implementation in deep-space communications \\
\hline 2011 & Zhang et al. [49] & $\begin{array}{c}\text { A tutorial on SuperPosition Coding aided RF communications and its applications in the } \\
\text { context of HARQ schemes }\end{array}$ \\
\hline 2013 & Chen et al. [50] & A survey and tutorial on HARQ-based turbo code schemes in $R F$ communications \\
\hline 2014 & Ngo et al. [51] & Current state-of-art of HARQ in the context of cooperative RF communications \\
\hline 2016 & Mukhtar et al. [52] & A survey on the integration of turbo product codes and HARQ in $R F$ communications \\
\hline 2018 & Jiang et al. [53] & An overview of ARQ and HARQ in underwater acoustic network (UWAN) \\
\hline 2020 & Makki et al. [54] & A survey of HARQ using NOMA for $R F$ communications \\
\hline 2021 & Ahmed et al. [55] & A survey of HARQ in $R F$ communications with various emerging wireless technologies \\
\hline & This paper & $\begin{array}{l}\text { A comprehensive survey on the design and performance evaluation of both ARQ and } \\
\text { HARQ protocols together with cross-layer design frameworks in various FSO } \\
\text { communication scenarios, including point-to-point terrestrial, cooperative, multi-hop } \\
\text { relaying, hybrid FSO/RF, satellite/aerial, and deep-space systems }\end{array}$ \\
\hline
\end{tabular}

in the FSO systems for two remarkable reasons. Firstly, as the bandwidth of FSO systems is much larger than that of RF ones, it is challenging for error-control methods designed for the lower data-rate transmission of RF systems to fully exploit such huge bandwidth. As a matter of fact, for example, the stop-and-wait (SW) ARQ protocol has been widely employed and performed well in RF communications [56]-[58]. This protocol, nevertheless, may not be suitable for high-speed FSO systems due to its inefficient bandwidth utilization, as reported in [34]. Secondly, as the FSO fading channel models are entirely different from those of RF, it would be crucial to have a proper protocol design under the atmospheric turbulence conditions in terms of system performance optimizations. As a result, it is of vital importance and necessity to provide an overview of current studies and tutorials associated with the design and performance of link-layer retransmission-based error-control protocols in the FSO communication systems.

\section{Article Contributions}

In this paper, our focus is, therefore, a comprehensive review of the design and performance evaluation of link-layer retransmission-based error-control protocols in the context of FSO communications. The main contributions of the paper can be summarized as follows.

1) It is an up-to-date review of link-layer retransmissionbased error-control protocols, both ARQ and HARQ, in various FSO communication scenarios, including pointto-point terrestrial, cooperative, multi-hop relaying, hybrid FSO/RF, satellite/aerial, and deep-space systems.

2) A survey and insightful discussion on the cross-layer design frameworks of link-layer retransmission protocols with PHY methods and/or transport layer protocols in the context of FSO communication networks are also provided.

3) From the holistic survey, we dedicate elaborated lessons learned section and outline future research directions to develop link-layer retransmission protocols and their cross-layer design frameworks in FSO communication networks.

4) Based on the lessons learned from this survey paper, we derive generic design guidelines recommended for effectively designing such protocols in each FSO network scenario.
5) Finally, we identify the important research challenges and discuss the open issues for such protocols in the vision of future sixth-generation $(6 \mathrm{G})$ wireless communication networks.

It is noteworthy that a highlight of our contributions compared to existing surveys relating to error-control methods in the context of FSO communications is illustrated in Table II. Additionally, the difference of our work compared to ones in RF communications is briefly summarized in Table III.

\section{Paper Organization}

The remainder of this paper is organized as follows. Section II presents state-of-the-art literature on ARQ protocols for different FSO communication scenarios, including point-topoint terrestrial, cooperative, hybrid FSO/RF, satellite/aerial, and deep-space systems, and then outlines future research directions. In Section III, we provide an extensive review of the HARQ protocols in various FSO systems, i.e., point-to-point terrestrial, cooperative, multi-hop relaying, hybrid FSO/RF, satellite/aerial, and then point out the potential research topics for such protocols. We dedicate Section IV to introduce and discuss cross-layer design frameworks related to the linklayer retransmission protocols, including the joint design of physical-layer/link-layer and link-layer/transport-layer in FSO communication networks. As a result of our extensive survey, Section V provides lessons learned, design guidelines, and discuss the challenges as well as open issues for link-layer retransmission protocols in FSO communications. Finally, Section VI concludes the paper. In addition, for the sake of explicit clarity, the organization of this paper is depicted in Fig. 2.

\section{ARQ aided FSO Communications}

The ARQ protocol, one of the most popular link-layer error-control schemes, is an efficient solution to increase link reliability. The advantages of ARQ protocols have been proven in RF-based wireless systems for a long time. Nevertheless, the high data-rate transmission in FSO systems over time-varying atmospheric turbulence channels poses new challenges to the design and performance of such protocols. A proper design of ARQ protocols for new/future immense-bandwidth FSO systems becomes an essential aspect of being considered. To 


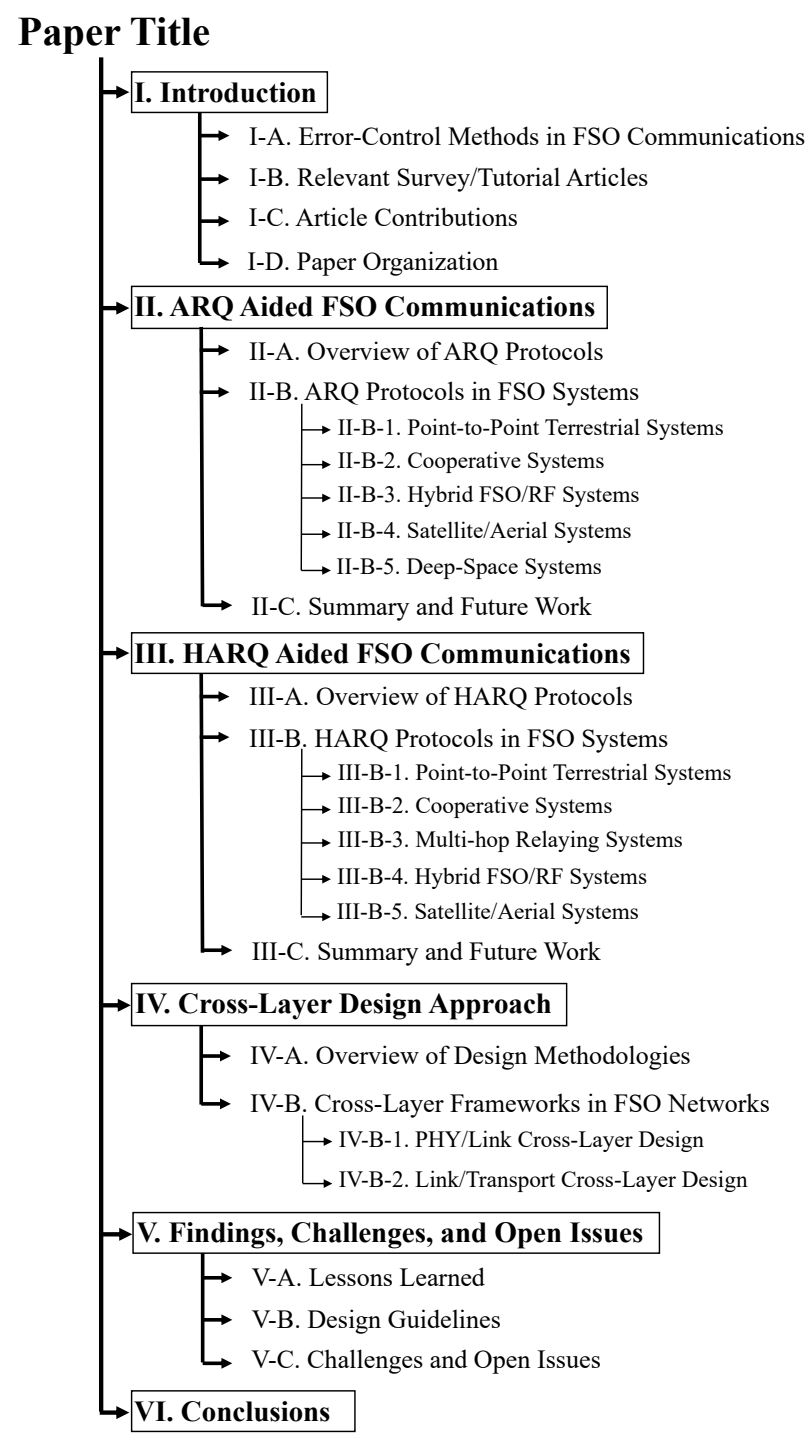

Figure 2: Organization of this paper.

this end, in this section, we first revisit the background of ARQ protocols. Next, we provide an overview of the state-ofart design and performance evaluation of ARQ protocols in various FSO communication scenarios covering from point-topoint terrestrial, cooperative, hybrid FSO/RF, satellite/aerial, to deep-space systems. Finally, we summarize the primary characteristics of reviewed ARQ protocols investigated in various FSO systems, followed by possible future directions for such protocols.

\section{A. Overview of $A R Q$ Protocols}

Background: The ARQ protocol provides a reliable transmission service for the link layer by detecting and retransmitting erroneous frames. More specifically, the transmitter sends a frame consisting of data and an error detection code, such as the standard cyclic redundancy check (CRC). The receiver can verify the integrity of the received frame using the error detection code. Then, based on the verification result, a feedback message, i.e., either a positive acknowledgment (ACK) or negative acknowledgment (NAK), is returned to the transmitter. The transmitter transmits a new frame if an ACK is received, whereas it retransmits the frame upon the receipt of the NAK message. The process continues until an ACK is received or a predefined number of retransmissions is reached. In case of missing frames or feedback messages, a timer is needed for a frame to activate the retransmission phase after a time-out duration.

(a) ARQ Classification

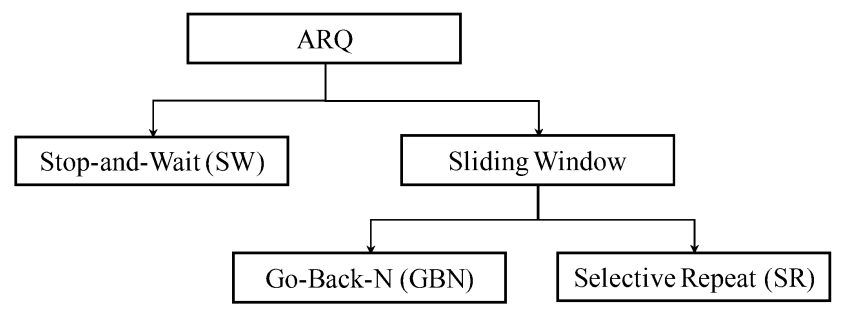

(b) Comparison of ARQ Types

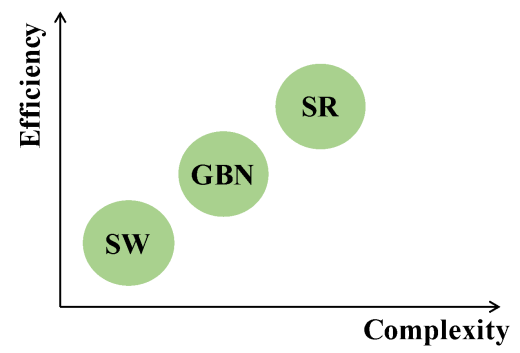

Figure 3: (a) ARQ Classification, and (b) Comparison of ARQ Protocols.

Classification: Based on the retransmission strategies, there are two basic types of ARQ schemes: stop-and-wait (SW) and sliding window protocols as depicted in Fig. 3(a). The sliding window ARQ is further categorized into go-back-N (GBN) and selective repeat (SR), and the operation of ARQ schemes can be briefly described as follows [59]:

- Stop-and-wait $A R Q$ : The transmitter sends a single frame at a time and waits for an ACK from the receiver side. If the ACK is received, a new frame will be transmitted. Otherwise, frame retransmission is activated.

- Go-Back-N ARQ: The transmitter sends multiple frames specified by a window size (denoted as $W$ in the number frames). The receiver keeps track of the frame index within the window size. When a frame's index is not as expected (i.e., out of order or duplicate), it will return a NAK message containing the index ACK for the last correct in-order frame. The transmitter starts retransmission of its entire window, commencing from the most recent positively acknowledged frame, and continues the process over again.

- Selective Repeat $A R Q$ : The data frames are continuously transmitted without waiting for acknowledgment from the receiver, as in the GBN-ARQ. However, unlike the GBN$A R Q$, the receiver accepts out-of-order frames and buffers them, and only missing/negatively acknowledged frames are retransmitted. 
Comparison between ARQ protocols: Of the three ARQ protocols, as illustrated in Fig. 3(b), SR-ARQ is able to achieve the highest transmission efficiency but its implementation is the most complicated. Further discussions on the comparison of ARQ protocols regarding the complexity and transmission efficiency are as follows.

- Complexity: The complexity comes from the protocol implementation, which is mainly related to (i) frame buffering and (ii) timer management. First, a buffer is required at the transmitter side for GBN-ARQ and SRARQ due to the transmission of multiple frames. In SWARQ, no transmitter's buffer is needed. Additionally, as the receiver accepts and keeps out-of-order frames, a receiver's buffer is required for the SR-ARQ. On the other hand, only one timer is necessary for each transmission round for SW-ARQ and GBN-ARQ. In contrast, multiple timers are required for each transmitted frame in the SRARQ. Therefore, it is clear that SR-ARQ is the most complicated protocol, while SW-ARQ is the simplest one in terms of implementation. The comparison of ARQ protocols concerning the complexity is summarized in Table IV

Table IV: Complexity comparison of ARQ protocols.

\begin{tabular}{|l|c|c|l|}
\hline ARQ Type & $\begin{array}{c}\text { Transmitter's } \\
\text { Buffer }\end{array}$ & $\begin{array}{c}\text { Receiver's } \\
\text { Buffer }\end{array}$ & Timer Required \\
\hline SW-ARQ & $\times$ & $\times$ & for one frame \\
\hline GBN-ARQ & $\checkmark$ & $\times$ & for one frame \\
\hline SR-ARQ & $\checkmark$ & $\checkmark$ & for all frames \\
\hline
\end{tabular}

- Transmission Efficiency: Transmission efficiency is defined as the ratio of useful data rate over the channel bit rate. The sliding window ARQ outperforms SWARQ in terms of transmission efficiency thanks to the transmission of multiple frames without waiting for an acknowledgment. This fact becomes clearer in the scenario of long fat networks (LFNs), as shown in Table V. In this example, we compare the transmission efficiency of ARQ protocols in high-speed and long-distance satellite communications. Also, the bit rate $R_{\mathrm{b}}=1 \mathrm{Gbps}$, satellite altitude $H_{\mathrm{s}}=600 \mathrm{~km}$, frame size of $n_{f}=1250$ bytes including $n_{o}=25$ bytes of overhead, ACK frame of $n_{a}=25$ bytes, window size $W=11$ frames, and different values of BER are taken into account. Here, $P_{f}$ is the frame error rate (FER), which is computed as $P_{f}=1-(1-\mathrm{BER})^{n_{f}}$. As seen, SW-ARQ is not practically useful in LFN as it retains a very low level of transmission efficiency. In addition, among sliding window protocols, SR-ARQ can achieve higher transmission efficiency than GBN-ARQ because only missing or negatively acknowledged frames are retransmitted in SR-ARQ.

\section{B. ARQ Protocols in FSO Systems}

The ARQ protocols have been widely studied for FSO communications, and several variants and modifications were adopted for reliable and very high-throughput systems. A summary of recent studies on ARQ protocols for various FSO system scenarios, including point-to-point terrestrial, cooperative, hybrid FSO/RF, satellite/aerial, and deep-space systems, is given in Table VI. The following provides further details on the design and performance estimation of ARQ protocols studied in such scenarios.

1) Point-to-point Terrestrial Systems: The performance of FSO terrestrial systems, e.g., building-to-building, severely deteriorated by the impact of atmospheric turbulence, leading to the fluctuations of optical signal intensity and phase [76]-[79]. This is one of the most challenging issues for the widespread deployment of the FSO in communication networks. Over the last decade, there have been many studies on error-control solutions focusing on the ARQ protocols to mitigate the transmission errors over turbulence-induced fading channels [33], [34], [61], [62], [68], [72], [73].

Early work on ARQ protocols for FSO communications was performed by Milner et al. in [61]. This study quantitatively compared two well-known link-layer error-control solutions, i.e., error correction code (ECC) schemes and ARQ protocols, over the turbulence fading channels. It was shown that for arbitrarily long-term outages, i.e., order of few seconds duration, the ARQ technique is more efficient in terms of throughput performance. On the contrary, the ECC scheme contains constant overhead, which is helpful for the worst-case situations. In addition, working with significant overhead designed for the worst-case situation by ECC schemes also introduces a long latency and low achievable goodput performance. This could be problematic for time-critical services, in which not only the link establishment but also service requirements dictate the design of proper error correction schemes. Combining error control schemes to offer a more robust error-control solution to tackle the issue would be necessary. For instance, the enhancement at the physical (PHY) layer using the FEC with a short coding block length (negligible delay) would reduce the number of retransmissions implemented by ARQ protocols at the link layer, thereby improving the latency and throughput performance. Indeed, the selection of error-control methods should be governed by the quality of service (QoS) requirements. Another comparison between the ECC scheme using Luby Transform (LT) codes and SR-ARQ protocol over the FSO turbulence fading channels was indicated in [62]. For various atmospheric turbulence conditions in FSO channels, the SR-ARQ protocol could retain a better throughput performance in weak turbulence regimes. At the same time, LT code outperforms the SR-ARQ scheme in moderate-tostrong turbulence regimes. The performance of ARQ protocols is degraded in error-prone environments, especially in strong turbulence conditions, as many retransmissions are required.

The above-mentioned studies addressed the ARQ protocols regarding performance estimation without introducing any novel designs in the context of FSO communications. The deployment of ARQ protocols over weak-to-strong turbulence channels would be beneficial and more effective if combined with PHY's error-control solutions, resulting in novel designs of such protocols in FSO systems. The adaptive rate (AR) transmissions employed at the PHY layer has been widely considered in the domain of FSO communications to increase the transmission rate while maintaining QoS requirements based on the turbulence channel variations [11]. The joint design between AR transmissions and ARQ protocols is very 
Table V: Transmission efficiency comparison of ARQ protocols in high-speed satellite communications [60, Section 5].

\begin{tabular}{|c|c|c|c|c|c|c|}
\hline \multirow{2}{*}{ ARQ Type } & \multirow{2}{*}{ Expression } & \multicolumn{5}{|c|}{ Bit error rate } \\
\hline & & $10^{-4}$ & $10^{-5}$ & $10^{-6}$ & $10^{-7}$ & $10^{-8}$ \\
\hline SW-ARQ & $\eta_{\mathrm{SW}}=\frac{1-\frac{n_{o}}{n_{f}}}{1+\frac{n_{a}}{n_{f}}+\frac{2\left(t_{\mathrm{prop}}+t_{\mathrm{proc}}\right) R_{\mathrm{b}}}{n_{f}}}\left(1-P_{f}\right)$ & $0.0899 \%$ & $0.2211 \%$ & $0.2419 \%$ & $0.2441 \%$ & $0.2444 \%$ \\
\hline GBN-ARQ & $\eta_{\mathrm{GBN}}=\frac{1-\frac{n_{o}}{n_{f}}}{1+(W-1)}\left(1-P_{f}\right)$ & $4.9240 \%$ & $45.4359 \%$ & $88.2444 \%$ & $96.9332 \%$ & $97.8923 \%$ \\
\hline SR-ARQ & $\eta_{\mathrm{SR}}=\left(1-\frac{n_{o}}{n_{f}}\right)\left(1-P_{f}\right)$ & $36.0504 \%$ & $88.6740 \%$ & $97.0249 \%$ & $97.9020 \%$ & $97.9902 \%$ \\
\hline
\end{tabular}

Table VI: Literature review of ARQ protocols in various FSO scenarios, including point-to-point terrestrial (PP), cooperative (CP), hybrid FSO/RF (HB), satellite/aerial (SA), and deep-space (DS) systems.

\begin{tabular}{|c|c|c|c|c|c|c|c|c|c|c|}
\hline \multirow{2}{*}{ Ref. } & \multirow{2}{*}{ Objective } & \multicolumn{3}{|c|}{ ARQ Type } & \multicolumn{5}{|c|}{$\begin{array}{l}\text { System } \\
\end{array}$} & \multirow{2}{*}{ Remarks } \\
\hline & & SW & GBN & SR & $\mathbf{P P}$ & $\mathbf{C P}$ & HB & SA & DS & \\
\hline [61] & FLR & & & $\checkmark$ & $\checkmark$ & & & & & $\begin{array}{l}\text { Very first work investigated ARQ protocols in the } \\
\text { context of FSO communications }\end{array}$ \\
\hline [62] & Throughput, FER & & & $\checkmark$ & $\checkmark$ & & & & & $\begin{array}{l}\text { A comparison between SR-ARQ and ECC using } \\
\text { LT codes in different turbulence conditions }\end{array}$ \\
\hline [63] & $\begin{array}{l}\text { Goodput, Delay, EE, } \\
\text { FER }\end{array}$ & $\checkmark$ & & & & $\checkmark$ & & & & $\begin{array}{l}\text { The proposed M-C-ARQ outperforms the } \\
\text { conventional C-ARQ in cooperative FSO systems }\end{array}$ \\
\hline [64], [65] & $\begin{array}{c}\text { Transmission } \\
\text { Efficiency, FER }\end{array}$ & $\checkmark$ & $\checkmark$ & $\checkmark$ & & & & $\checkmark$ & & $\begin{array}{l}\text { An investigation of ARQ protocols in } \\
\text { long-distance HAP-to-HAP FSO links }\end{array}$ \\
\hline$[66],[67]$ & $\begin{array}{l}\text { Goodput, Outage } \\
\text { Probability, FER }\end{array}$ & $\checkmark$ & & & & & $\checkmark$ & & & $\begin{array}{l}\text { Analysis of SW-ARQ in adaptive multi-rate } \\
\text { hybrid FSO/RF systems }\end{array}$ \\
\hline [33], [68] & $\begin{array}{c}\text { SE, Outage } \\
\text { Probability, FER }\end{array}$ & $\checkmark$ & & & $\checkmark$ & & & & & $\begin{array}{l}\text { Two cross-layer designs are considered: standard } \\
\text { AR/ARQ and AR/ARQ with FC }\end{array}$ \\
\hline [69] & $\begin{array}{l}\text { Throughput, Delay, } \\
\text { FLR }\end{array}$ & & & $\checkmark$ & & & & & $\checkmark$ & $\begin{array}{l}\text { An investigation of disruption-tolerant SR-ARQ } \\
\text { protocols in the context of deep-space systems }\end{array}$ \\
\hline [70] & Throughput & & & $\checkmark$ & & & & $\checkmark$ & & $\begin{array}{l}\text { Study the tradeoff between ARQ feedback rate } \\
\text { and ARQ protocol efficiency in satellite systems }\end{array}$ \\
\hline [71] & $\begin{array}{l}\text { Throughput, Outage } \\
\text { Probability }\end{array}$ & $\checkmark$ & & & & & $\checkmark$ & & & $\begin{array}{c}\text { Evaluate the effect of adaptive power allocation } \\
\text { between the ARQ retransmissions }\end{array}$ \\
\hline $\begin{array}{c}{[34],} \\
{[72],[73]}\end{array}$ & $\begin{array}{l}\text { Throughput, Delay, } \\
\text { FER }\end{array}$ & & $\checkmark$ & $\checkmark$ & $\checkmark$ & & & & & $\begin{array}{l}\text { Highlight the cross-layer design of sliding } \\
\text { window ARQ and AR in FSO systems }\end{array}$ \\
\hline [74] & Throughput & & & $\checkmark$ & & & & $\checkmark$ & & $\begin{array}{l}\text { Experimentally demonstrate the use of SR-ARQ } \\
\text { protocol in FSO-based satellite systems }\end{array}$ \\
\hline [75] & $\begin{array}{l}\text { Goodput, Outage } \\
\text { Probability, FER }\end{array}$ & $\checkmark$ & & & & & $\checkmark$ & & & $\begin{array}{l}\text { Analysis of SW-ARQ in hybrid FSO/RF systems } \\
\text { with FSO links described by Malaga model }\end{array}$ \\
\hline
\end{tabular}

attractive to counteract the transmission errors without using costly FEC schemes at the PHY layer. Integration in the design of standard SW-ARQ protocol and AR scheme for FSO system was studied in [68]. This design offers a considerable system performance improvement in terms of spectral efficiency (SE) and average FER compared to the conventional design of the alone ARQ protocol or alone AR scheme. For such joint design, another solution to increase the reliability of standard SW-ARQ protocol is the proposal of frame combining (FC) ARQ scheme realized by implementing frame combining at the receiver side in [33]. For SW-ARQ with FC, the receiver can store all copies of previously erroneous frames to jointly decode with retransmission ones. This improves the likelihood of successfully retransmitting frames. In practical systems, as the receiver always has a buffer to store frames before delivering them to end-users, this solution does not introduce additional system costs. In addition, the SW-ARQ with FC performs more efficiently than standard SW-ARQ in different turbulence conditions.

It is worth noting that the SW-ARQ protocol is practically not useful, especially in point-to-point high-speed systems, due to its inefficient bandwidth utilization. Practical systems employ the sliding window protocols, i.e., GBN and SR, which allow higher efficiency. Driven by that fact, the design of sliding window ARQ protocols, both GBN and SR mechanisms, with ARQ burst transmissions was investigated in [34], [72], [73]. Regarding the design of such protocols, one of the critical issues is the time-varying behavior of turbulence fading channels. For FSO links operating at high data rates, as the temporal coherence time of the atmospheric turbulence process is of the order of tens of milliseconds, the error probability of frames are highly correlated, and frame errors tend to happen in bursts [80], [81]. This issue, however, was not mentioned for the design of FSO systems using SW-ARQ as a single 
frame is transmitted per round trip delay. As a result, due to the transmission of multiple frames, the window size of sliding window ARQ protocols, which is based on the burst duration, was designed considering the time-varying behavior of the turbulence-induced fading channels [34]. As is evident, the existing studies demonstrated the outperformance of sliding window ARQ protocols compared to the SW-ARQ one in high-speed FSO communications.

2) Cooperative Systems: Cooperative diversity has been widely studied for FSO communications to realize spatial diversity advantages [82]-[84]. The key idea behind cooperative diversity is based on observing the FSO turbulence channels. The signal transmitted by a source node is overheard by others nodes, i.e., called relay nodes. This introduces additional degrees of freedom in the spatial domain, thus significantly reducing the impact of atmospheric turbulence. For cooperative FSO systems, parallel relaying can be implemented through the use of multiple transmitter apertures directed to relay nodes, as shown in Fig. 4.

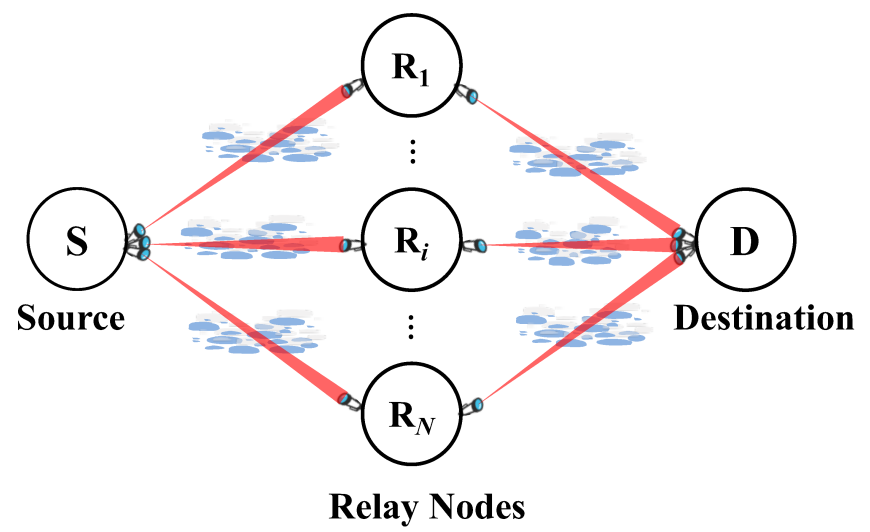

Figure 4: An FSO system model for cooperative ARQ protocols over atmospheric turbulence channels.

In addition, as reported in [85], the cooperation through relay nodes is beneficial only if the received signal-to-noise ratio (SNR) is high enough; otherwise, relay nodes likely forward the corrupted copies of data, leading to performance deterioration. To tackle the issue, ARQ protocols can be employed to improve the performance of such cooperative FSO systems. Indeed, an effective design of ARQ protocols, together with the benefit of cooperative diversity, could offer significant performance enhancement for FSO systems.

The design and performance evaluation of cooperative ARQ (C-ARQ) protocol was first reported in [63], in which the SW mechanism was used for C-ARQ. Figure 4 illustrates an FSO system model for C-ARQ protocol, where the communication between a source (S) node and a destination (D) node is achieved through $N$ relay nodes $\left(\mathrm{R}_{i}, 1 \leq i \leq N\right)$ placed between the $\mathrm{S}$ node and the $\mathrm{D}$ node. This study mentioned two design approaches, including conventional C-ARQ and modified CARQ (M-C-ARQ) schemes. Their performance comparison was investigated in FER, Goodput, delay, and energy efficiency (EE). In the conventional C-ARQ, the S node broadcasts a data frame to all $\mathrm{R}$ nodes for the first transmission. These $\mathrm{R}$ nodes, which can successfully decode the data from $\mathrm{S}$, forward the frame to D. At the destination side, if the frame is successfully received by the D node, it will send an ACK message to the $\mathrm{S}$ node through $\mathrm{R}$ nodes; otherwise, a NAK message is sent. The failure occurs when either no $\mathrm{R}$ nodes can decode the frame successfully or the presence of errors is detected by $\mathrm{CRC}$ at the $\mathrm{D}$ node. In that case, the $\mathrm{S}$ node will retransmit that frame until reaching a predefined maximum number of retransmission attempts for a frame, i.e., $N_{\mathrm{t}}$ times. If a frame does not get through the FSO links after the ARQ's persistent level of $N_{\mathrm{t}}$ transmission attempts, the $\mathrm{S}$ node gives up, and that frame is clarified to be lost.

In the conventional $\mathrm{C}$-ARQ protocol, $\mathrm{R}$ nodes play the role of forwarding the frames to the $\mathrm{D}$ node. In case of transmission failure, the $\mathrm{S}$ node has to re-broadcast frames to all $\mathrm{R}$ nodes, including those successfully received by $\mathrm{R}$ nodes before. This leads to additional delay and energy consumption, especially in strong turbulence conditions. The M-C-ARQ scheme was proposed to address this issue [63]. Specifically, $\mathrm{R}$ nodes are equipped with buffers that can store the copy frames. Successful R nodes keep a copy of the frame for the first transmission round before forwarding it to the $\mathrm{D}$ node. If a NAK message arrives, $R$ nodes could retransmit the frame to the $\mathrm{D}$ node. As a result, the broadcast of retransmitted frames from the $\mathrm{S}$ node to all $\mathrm{R}$ nodes is no longer needed, thus improving system performance.

Figure 5 shows the performance comparison of conventional C-ARQ and M-C-ARQ over FSO turbulence fading channels. The transmitted powers vary when the number of relay nodes $N=2$, ARQ's persistent level $N_{\mathrm{t}}=4$, the turbulence strength $C_{n}^{2}=10^{-14} \mathrm{~m}^{-2 / 3}$, and the distance from the $\mathrm{S}$ node to the $\mathrm{D}$ node as $l_{\mathrm{SD}}=3000 \mathrm{~m}$. It is noted that interested readers can refer to the study in [63] for the details of derived equations for obtained results. In addition, several performance metrics, including FER, Goodput, and EE, are investigated in Figs. 5a, 5b, and 5c, respectively. As is expected, the M-CARQ protocol offers better performance than the C-ARQ one over FSO turbulence fading channels.

3) Hybrid FSO/RF Systems: The FSO system is severely affected by several factors, including atmospheric turbulence, strong attenuation by dense fogs and low clouds, pointing errors and misalignment on dynamic links, etc. These adverse issues lead to frequent link outages and dramatic degradation of system performance. One of the effective solutions is the hybrid system in which an RF link is used in parallel with the FSO link, which serves as a backup link in case of FSO link failure [86]. Such an RF link is less subject to the atmospheric turbulence and pointing misalignment [87], and is also much less affected by fogs and clouds [88]. For example, the impact of fog and rain on the corresponding FSO and RF links is drastically, but these factors rarely happen simultaneously [37]. As a result, the two links can function in a complementary manner.

Driven by the potential of a hybrid FSO/RF system, there have been several studies on the performance evaluation of ARQ protocols for such system [66], [67], [71], [75]. It is worth noting that the challenge on the design of ARQ protocols for hybrid $\mathrm{FSO} / \mathrm{RF}$ systems comes from the fact that the corresponding data rate in the RF channel is slower 


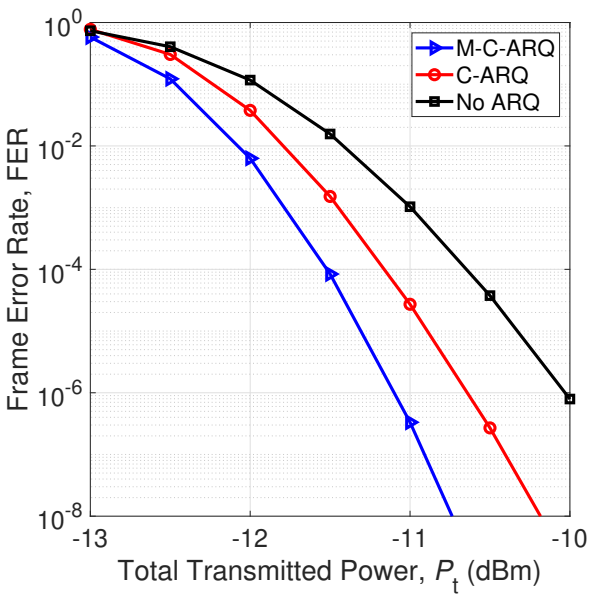

(a) Frame Error Rate

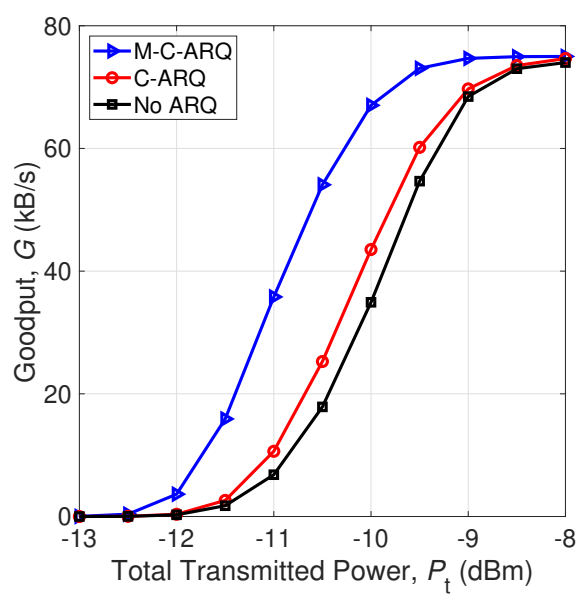

(b) Goodput

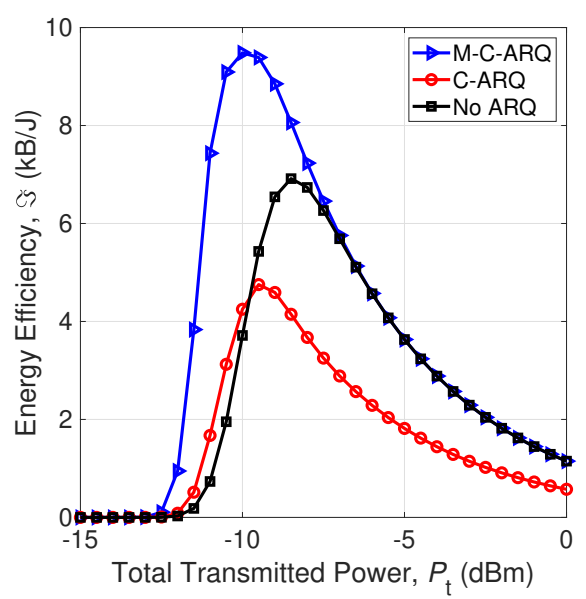

(c) Energy Efficiency

Figure 5: Performance comparison of conventional C-ARQ and M-C-ARQ [63].

than that in the main FSO link. Moreover, the relative fading channel coherence time for such two links is different, e.g., typically on hundreds of microseconds for millimeter-Wave (mmWave) links [89] and tens of milliseconds for FSO links [90]. Therefore, there is a critical need to design ARQ protocols considering those issues properly.

In many existing studies of ARQ protocol, the SW mechanism is often used thanks to its analysis simplicity. Notably, the performance of the SW-ARQ protocol was analyzed in adaptive multi-rate hybrid FSO/RF systems [66], [67]. In such scenarios, the design of rate adaptation allows switching between two links gradually to reduce the frequent link switching in error-prone environments of conventional fixedrate design, thus improving the system performance. In the design of ARQ protocol handling one frame at a time, its frame size is adjusted accordingly to transmission modes with different data rates to satisfy a predefined link-layer FER. Compared to the conventional fixed-rate design, a considerable enhancement of ARQ performance in adaptive multi-rate hybrid FSO/RF systems was confirmed [66], [67]. Besides the popular Gamma-Gamma model for FSO turbulence channel [66], [67], the Malaga distribution model is also used [75]. This comprehensive model for a wide range of turbulence conditions offers a more accurate SW-ARQ protocol performance estimation in adaptive multi-rate hybrid FSO/RF systems.

In [71], Makki et al. investigated the adaptive power allocation for ARQ retransmissions in the fixed-rate design of hybrid FSO/RF systems. In such systems, the ARQ frames in FSO and RF modes are simultaneously transmitted to the receiver. These frames are then combined and jointly decoded at the receiver side. Instead of using the same power for each retransmission, the basic idea for adaptive power allocation is to weigh the energy in each ARQ's retransmission round by its consumption probability. Additional energy is assigned to the last retransmissions, which are rarely used. This results in a significant improvement in system performance.

4) Satellite/Aerial Systems: The advancement in space technology and the development of sophisticated space-based instruments have opened a new chapter for space-based FSO communications [91], [92]. Researchers have been actively working on building all-optical communication systems for the demand of high-speed connectivity and large coverage area, e.g., FSO connections from satellites to ground stations, for inter-satellite, or between high platform altitudes (HAPs) [38]. It is worth noting that the challenging issues involved in space-based FSO links are different in different scenarios. In particular, the major adverse issue on the inter-satellite FSO links, which is at the altitudes of hundreds of kilometers in the absence of atmospheric turbulence and clouds, is the pointing misalignment due to the narrow laser beam pointing from/to the moving platform. For satellite-to-ground systems, the critical concerns of FSO links are the atmospheric turbulence, pointing errors, and cloud coverage [88]. As a result, understanding the impact of adverse issues on different spacebased FSO communication systems play an essential role in the system design and performance optimizations.

For reliable satellite/aerial communications, in addition to ECC schemes, ARQ protocols have been investigated for interHAPs [64], [65] and satellite [74] systems. The link distances of such communication systems are typically a few hundreds of kilometers, resulting in high latency [93]. Therefore, the biggest challenge on the design of ARQ protocols is the satisfactory delay and throughput performance, as they require retransmissions for erroneous frames under the time-varying turbulence fading channels. The frequent retransmissions also degrade the system energy efficiency, which is especially important for the limited power budget of satellite/aerial-based FSO communications. In addition, as mentioned earlier, an ARQ scheme cannot be designed generically for all FSO applications. They have to be tailored for each specific network scenario experienced with different challenging issues.

The use of ARQ protocols for FSO-based inter-HAP communications was addressed by the German Aerospace Centre (DLR) [64], [65]. Notably, the performance of ARQ variants, including SW, GBN, and SR, was estimated under the combined impact of pointing error and atmospheric turbulence. 
Table VII: Selected ARQ schemes with minimum required FER to maintain optimal TE in different scenarios [64].

\begin{tabular}{|c|c|c|c|c|}
\hline Inter-HAP Scenario & $\begin{array}{c}\text { Link } \\
\text { Distance }\end{array}$ & \multicolumn{3}{|c|}{ Required FER } \\
\cline { 3 - 5 } & $\begin{array}{c}\text { SW- } \\
\text { ARQ }\end{array}$ & $\begin{array}{c}\text { GBN- } \\
\text { ARQ }\end{array}$ & $\begin{array}{c}\text { SR- } \\
\text { ARQ }\end{array}$ \\
\hline Short Link - Low SI & $220 \mathrm{~km}$ & $\times$ & $10^{-5}$ & $10^{-2}$ \\
\hline Short Link - High SI & $339 \mathrm{~km}$ & $\times$ & $10^{-5}$ & $10^{-2}$ \\
\hline Long Link - Low SI & $426 \mathrm{~km}$ & $\times$ & $10^{-5}$ & $10^{-2}$ \\
\hline Long Link - High SI & $516 \mathrm{~km}$ & $\times$ & $10^{-5}$ & $10^{-2}$ \\
\hline
\end{tabular}

The results obtained in these studies allow selecting proper ARQ variants for different inter-HAP scenarios based on the channel conditions. As depicted in Table VII, based on the minimum FER required to retain maximum TE performance, a suitable ARQ scheme could be chosen for different link distances and atmospheric turbulence conditions indicating by the scintillation index (SI) [64]. As seen, the SW-ARQ protocol, the simplest and easiest to implement, fails in these scenarios due to the deficient achievable TE performance over long-distance turbulence channels. In addition, the minimum required FERs to maintain the optimal TE performance for GBN-ARQ and SR-ARQ protocols are respectively $10^{-5}$ and $10^{-2}$ in different turbulence conditions. This confirms that the effect of turbulence is generally small at the HAP's altitude, i.e., $20 \mathrm{~km}$, resulting in the same FER level requirement. For the transmission of large "bandwidth-delay product" systems over noisy channels, GBN-ARQ may not be suitable. This is because the whole transmission window has to be retransmitted for any lost events using much channel capacity, reducing TE performance. The SR-ARQ protocol, which overcomes the inefficiency of GBN-ARQ one by retransmitting only erroneous frames, is more complex in terms of implementation due to its non-sequential storage management at the receiver. As a result, it is essential to switch between these two ARQ protocols for different system scenarios.

Another critical issue on the design of ARQ protocols is the capability to support extremely high data rate transmissions, on the order of tens of Gbps up to Tbps. NASA's Terabyte Infrared Delivery (TBIRD) program plans to demonstrate the capability of delivering multiple terabytes during short passes from a low Earth-orbit (LEO) satellite to a low-cost ground terminal [94]. These data volumes can be achieved by using very high-rate (i.e., the order of hundreds of Gbps) direct-toEarth FSO communication links. For such high-speed FSO connectivity, if the ARQ protocol is adequately designed, the resulting communication system will be genuinely error-free. In [74], Schieler et al. successfully demonstrated the errorfree FSO communications using SR-ARQ protocol at the data rate close to $100 \mathrm{Gbps}$ in various atmospheric turbulence conditions. To achieve that extremely high data rate, the ARQ's frame size is designed to be as large as $10 \mathrm{MB}$ so that the window size is shorter and close to the fading channel coherence time, which varies from $1 \mathrm{~ms}$ to 100 ms (a condition generally observed at night). In addition, due to the limitation of feedback channel capacities in FSObased satellite communications, the tradeoff between ARQ's feedback rate and its throughput performance was investigated in [70].

5) Deep-space Systems: The demand for high data rate transmissions is expected in future deep-space missions (on Mars, Mercury, etc.), which enables a wide range of services to support remote human operations [48]. With the rapid development of deep space exploration, a new era of study on FSO communications in deep space is of widespread concern, thanks to its high-speed connectivity and low power consumption compared to the RF counterpart [95]. Several missions on the optical communication test and space exploration have been conducted with an FSO deep space communication system (see references therein [96]). Nevertheless, various adverse issues, such as path loss, misalignment errors, atmospheric turbulence, and coronal solar wind turbulence, pose various challenges to the FSO system design for deep space missions [97]. Ensuring reliable communications is one of the primary requirements for future deep space FSO communication networks [98]. Indeed, the selection of proper errorcontrol methods plays an essential role in such systems with long propagation delay, limited onboard storage, and timeconstrained visibility window between spacecraft and ground stations. One of the reliability options currently available for FSO deep space systems is the use of ARQ protocols reported by NASA in [69].

In [69], Clare et al. investigated the FSO systems for envisioned deep space mission scenarios, in which the reliability is assured by data retransmissions of ARQ protocols. The SRARQ protocol is considered for the FSO communication with a single Earth ground station, as simpler ARQ variants may suffer significant performance deterioration with the large "delaybandwidth product" of deep space systems. Here, reliable RF-based communication is used for the feedback channel. The disruption-tolerant ARQ, such as Licklider Transmission Protocol (LTP) [99] of the Disruption-Tolerant Networking DTN suite [100], is assumed, in which ARQ process is aware of the link schedule with the delay prediction and correspondingly adjusts timeout timers.

Initially, when the link is active, frames are transmitted from an active queue by the spacecraft. These frames are then moved from the active to pending queue, and a timer is set to the RTT plus a short processing/guard time. The timer for frame transmissions is adjusted in each session of LTP, which is based on the delay prediction [99]. ACK message is transmitted back to the spacecraft for each successfully received data frame, which removes that frame from the pending queue. Otherwise, the data frames are moved back to the active queue and retransmitted if a time-out occurs. Such frames are placed at the beginning of the ARQ (receiving transmission priority) to minimize the latency variance. Clearly, for such FSObased deep space systems, the deployment of ARQ protocols strongly affects the latency performance, as erroneous data frames require a random number of retransmission attempts until they are successfully received. Such delay may also reorder the arrival of data units, which may impose additional delay for the high-layer information context. For example, the receiver must wait until all data frames forming a single TCP segment are received (TCP segment-level latency). 


\section{Summary and Future Work}

This section reviewed state of the art on design and performance evaluation of ARQ protocols in different FSO system scenarios. The main findings of this section can be summarized as follows.

- Most of the existing works in FSO-based terrestrial systems, i.e., point-to-point, cooperative, and hybrid FSO/RF communications, focussed on the design and performance evaluation of SW-ARQ protocol for the sake of simplicity in the analysis. In addition, the SW-ARQ protocol with the concept of frame combining [33] was shown to be more efficient than the standard SW-ARQ one in terrestrial FSO communications.

- Sliding window protocols, including GBN-ARQ and SRARQ, which have been recently investigated in pointto-point terrestrial FSO systems [34], [72], [73], were shown the potential for high-speed FSO communications by promising obtained results.

- Many studies showed exciting results to confirm the effectiveness of FSO communication systems integrating the design of adaptive multi-rate schemes and ARQ protocols, e.g., point-to-point terrestrial [33], [34] and hybrid FSO/RF [67], [75] systems.

- The SR-ARQ protocol, thanks to offering higher achievable throughput performance than other variants, was widely applied for long-distance communications of satellite/aerial as well as deep space systems. While only throughput performance was investigated, other crucial performance metrics for such systems, including delay and energy efficiency, are still lacking in the literature.

- Table VI summarizes the existing works on ARQ protocols in different FSO systems.

From the viewpoint of current issues addressed on the design of ARQ protocols in FSO communications, some potential future research directions can be further investigated, for example,

- Taking advantages of frames combining, adaptive multirate, and sliding window mechanisms, a novel design of ARQ protocols, a challenge but interesting, would offer better performance than SW-ARQ with FC [33] or sliding window protocols without FC [34] in FSO communications.

- While the M-C-ARQ protocol can achieve better performance than conventional C-ARQ one in cooperative diversity FSO systems [63], its operation is still based on the SW mechanism, which is not an optimal design for ARQ protocols in high-speed systems as pointed out in [34]. Sliding window mechanisms handling multiple frames at a time should be considered for designing such an M-C-ARQ protocol.

- The existing design of ARQ protocols in hybrid FSO/RF systems was based on SW mechanism and simultaneous/parallel FSO and RF transmissions. As reported in [101], the use of parallel FSO and RF transmissions, which wastes the system power, is not an efficient solution for hybrid FSO/RF systems. The switching schemes, where either the FSO or RF link is selected based on channel conditions, provide efficient transmission and higher data rate compared to the simultaneous one [102]. Indeed, addressing the design of sliding window ARQ protocols in switching-based hybrid FSO/RF systems considering the relative coherence times and data rates of two links would be challenging but increase the system performance.

\section{HARQ AIDED FSO COMMUNICATIONS}

The literature on the ARQ protocol revealed that it may fail to provide acceptable latency and throughput performance in some network scenarios, e.g., in long-distance FSO systems, due to many frame retransmissions required over time-varying turbulence channels. A more robust error-control method, i.e., hybrid ARQ (HARQ), which offers better reliability by combining ARQ and error correction code (ECC), is preferable to standard ARQ protocols in such scenarios. In this section, we first briefly present the background of HARQ protocols. Then, we review existing studies on the design and performance evaluation of HARQ for FSO communications in various scenarios, including point-to-point terrestrial, cooperative, multihop relaying, hybrid FSO/RF, and satellite/aerial systems. Finally, we summarize the main issues of reviewed HARQ protocols studied in different FSO system scenarios, followed by future research directions.

\section{A. Overview of HARQ Protocols}

Background: The ARQ protocol can be combined with the ECC to enhance the achievable ARQ efficiency over timevarying wireless fading channels. Such a combination of the two basic error-control schemes is referred to as hybrid ARQ (HARQ), which was introduced in the 1960s by Wozencraft and Horstein [103], [104]. Unlike the ARQ, the HARQ protocol can do both frame correction and retransmission of uncorrectable frames. In particular, the receiver handles error detection, error correction, and retransmission requests simultaneously. Retransmission is requested only when the receiver detects an uncorrectable error. Furthermore, the receiver can also store previously received frames for the joint decoding with the last received frame to improve decoding reliability. By combining error correction and retransmission and appropriately selecting an ECC scheme, the HARQ protocol can offer higher reliability than an ECC system alone and better throughput performance than a system with pure ARQ over time-varying turbulence fading channels.

Classification: HARQ protocols can be mainly classified into two categories [47], [51], namely type-I (TI) and type-II schemes, as shown in Fig. 6(a). Compared to the TI scheme, type-II is an advanced form of HARQ, which uses the concept of frame combining. The critical idea of frame combining is that even if a received signal detection fails, it still contains some useful information about the transmitted frame. Therefore, it is stored in the receiver buffer to be combined with other retransmissions, resulting in enhanced detection performance. Depending on the retransmission scheme and the combining method, type-II HARQ can be further partitioned into chase combining (CC) based on the retransmission of the 
(a) HARQ Classification

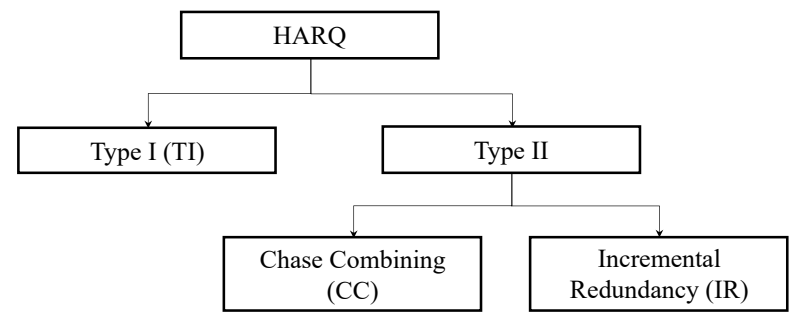

(b) Comparison of HARQ Types

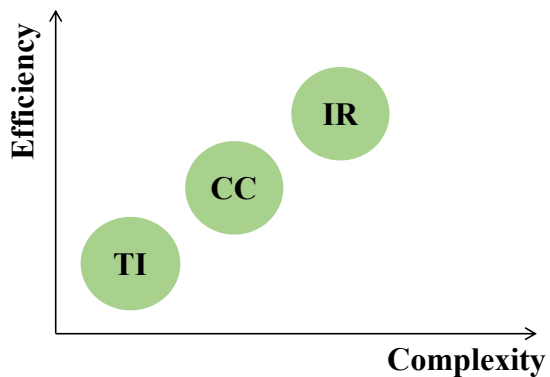

Figure 6: (a) HARQ Classification, and (b) Comparison of HARQ Protocols.

same coded frame; and incremental redundancy (IR), which is based on the retransmission of additional redundancy bits [105]. In the following, we give a brief description of HARQ schemes.

- Type I HARQ: The straightforward combination of ARQ and ECC is referred to as TI-HARQ. Early HARQ schemes designed in the 1970s and early 1980s [106][110] belong to the family of TI-HARQ, and this scheme has also been widely applied in wireless systems, e.g., [111]-[113]. More explicitly, an ECC-encoded frame is the original one complemented by parity bits. This frame is (re)transmitted for all (re)transmissions. All corrupted frames are discarded at the receiver side, and each ECC decoding action is performed solely for a single encoded frame. As reported in [114], the TI-HARQ is capable of improving the achievable throughput performance of conventional pure ARQ in a relatively high error-rate environment, as its error-correction capability reduces the frequent retransmissions. Nevertheless, if the channel conditions are sufficiently good, e.g., the BER below $10^{-4}$ [114, Fig. 1], the resultant reduced number of errors may not require any ECC parity bits. In this case, the throughput performance of the system using TI-HARQ is reduced by the unnecessary parity bits and becomes lower than that of conventional ARQ.

- Chase Combining HARQ: The CC-HARQ was proposed by D. Chase [115] for combining an arbitrary number of erroneous frames in a single frame based on a maximum likelihood criterion. In CC-HARQ, the modulated bits of the frame $\mathbf{x}$, which is created from the coded bits $\mathbf{c}$, is initially transmitted to the receiver. If the receipt of a NAK is confirmed, the same bits of frame $\mathbf{x}$ is retransmitted. This process for the retransmission of the same frame is continued until it is decoded successfully or reaches a predefined maximum number of transmission attempts of a frame, denoted by $M$. Then, the previously received copies of the same frame are combined at the receiver in a single signal, which contains the accumulated information of the transmitted frame from all received signals.

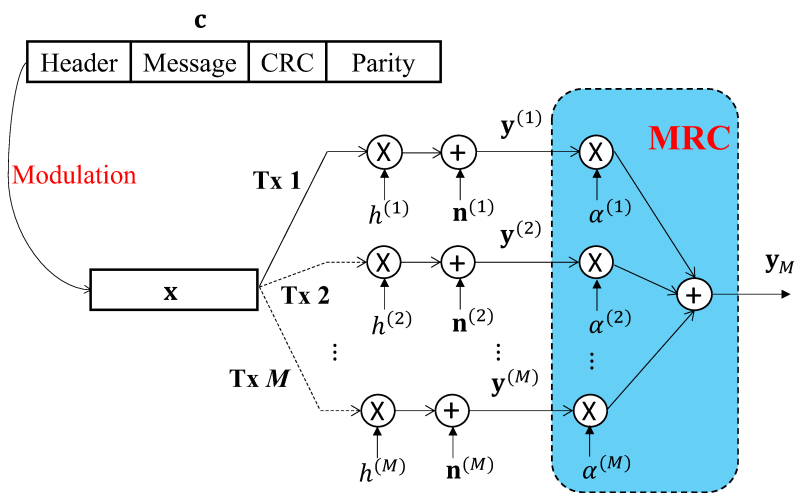

Figure 7: Chase combining HARQ scheme.

The operation of CC-HARQ with maximum-ratio combining (MRC) method is illustrated in Fig. 7, in which HARQ persistent is given by $M$. The received signal at the $i$-th transmission, denoted by $\mathbf{y}^{(i)}$, is expressed by

$$
\mathbf{y}^{(i)}=h^{(i)} \mathbf{x}+\mathbf{n}^{(i)},
$$

where $\mathbf{x}$ is the transmitted signal, $h^{(i)}$ is the channel fading coefficient, and $\mathbf{n}^{(i)}$ is signal-independent additive white Gaussian noise with variance $\sigma_{\mathrm{n}}^{2}$. After the $K$-th HARQ round $(K \leq M)$, the receiver combines the $K$ received frames at the symbol level in a single frame denoted by $\mathbf{y}_{K}$. The combined frame is obtained by weighting each frame by an estimation of its reliability before being summed with the other frames, i.e.,

$$
\mathbf{y}_{K}=\sum_{i=1}^{K} \alpha^{(i)} \mathbf{y}^{(i)}
$$

where $\alpha^{(i)}$ is the reliability of each received frame, which is given under maximum-likelihood decoding [116]. The CC-HARQ takes advantage from the accumulated SNRs of the individual transmissions to enhance the decoding performance of the transmitted frame.

- Incremental Redundancy HARQ: The IR-HARQ scheme, as depicted in Fig. 8, generalizes the CC-HARQ by considering that each transmission is a punctured version of a low rate mother FEC code, denoted by $C_{0}$. In IR-HARQ, the information frame, which includes frame header, message, and CRC, is first encoded by the original rate $C_{0}$ encoder (called mother code).

For the first transmission, only some coded bits of $C_{0}$ are chosen according to a predetermined puncturing pattern $\mathbf{P}_{1}$. The selected bits are grouped in a single block $\mathbf{c}^{(1)}$ which is modulated and then transmitted to the receiver side. Here, we denote the transmitted codeword 


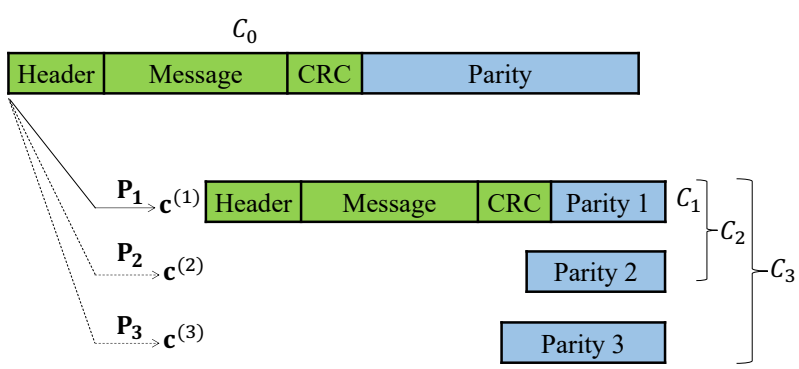

Figure 8: Incremental redundancy HARQ scheme.

by $C_{1}=\mathbf{c}^{(1)}$. At the receiver side, after decoding for error correction with code $C_{1}$, error detection using CRC is performed. If the presence of errors is detected, IRHARQ only retransmits the additional redundancy for uncorrectable frames based on a NAK message from the receiver. Accordingly, the transmitter sends the second block $\mathbf{c}^{(2)}$ containing the additional coded bits taken from the remaining bits of $C_{0}$, which have not been transmitted yet. The newly received redundancy is combined with the previously received frames to construct coded bits of code $C_{2}=\left[\mathbf{c}^{(1)}, \mathbf{c}^{(2)}\right]$ for decoding. This process for retransmission of a frame is continued until it is decoded successfully or reaches a predefined maximum number of transmission attempts of a frame $M$. Thus, we have $C_{1} \subseteq$ $C_{2} \subseteq \cdots \subseteq C_{M} \subseteq C_{0}$ which expresses the rate-compatibility restriction of the punctured codes in IR-HARQ protocols.

Comparison between HARQ protocols: Of the three HARQ protocols, TI-HARQ is the most straightforward implementation but provides a deficient throughput performance over time-varying fading channels. The CC and IR-HARQ protocols outperform the TI-HARQ thanks to the concept of frame combining. The IR-HARQ protocol can achieve better performance than the CC-HARQ because only incremental redundancies are retransmitted for each event of the erroneous frame. However, the CC-HARQ offers a lower complexity than IR-HARQ. This is because the use of IR-HARQ protocol requires additional signaling and a large-size buffer [117]. Additionally, since each retransmission is identical, the CCHARQ can easily combine with other techniques, e.g., spacetime coding and coded modulations. Moreover, as reported in [118], the gain of the IR-HARQ scheme compared to the CCHARQ one is more significant with higher code rates, while with the lower code rate, the performance of the CC-HARQ protocol is almost as good as that of IR-HARQ.

\section{B. HARQ Protocols in FSO Systems}

The design of HARQ protocols, which were widely investigated in the RF-based communication systems, has recently extended to the context of FSO communications. A summary of existing studies on HARQ protocols addressed in various FSO scenarios, including point-to-point terrestrial, cooperative, multi-hop relaying, hybrid FSO/RF, and satellite/aerial systems, are given in Table VIII. In the following, we aim to review works on HARQ protocols in each FSO communication system in detail.
1) Point-to-Point Terrestrial Systems: In the domain of point-to-point terrestrial FSO communications, there have been many studies on the design of HARQ protocols to mitigate the transmission errors caused by adverse issues, such as atmospheric turbulence and pointing errors [35], [36], [119], [122], [125], [129]. In these studies, the SW mechanism was used for HARQ for the sake of analysis simplicity. In addition, type II HARQ protocols, including CC and IR schemes, offered better performance than TI-HARQ one, were also considered in the existing studies for point-to-point terrestrial FSO systems. Notably, the very first work on HARQ protocols for FSO systems was reported in [119]. In this study, the authors addressed an IR-HARQ design with TrellisWare's flexible low-density parity-check (LDPC) code family in the presence of atmospheric turbulence. A family code implies the rate compatible, in which high-rate codewords can be obtained from the low-rate ones via the puncturing technique. Initially, the IR-HARQ protocols operate by transmitting a high-rate codeword. In the event of a NAK, the additional (i.e., punctured) parity bits required to form lower-rate codewords are subsequently transmitted.

A common approach to analyzing the performance of HARQ protocols in FSO systems is based on an informationtheoretic point of view. Kiasaleh et al. evaluated the performance of CC-HARQ in FSO systems using a pulseposition modulation and direct-detection mechanism [35], [125]. Moreover, similar to the traditional CC mechanism, which explores temporal diversity, the authors also considered a case where retransmissions are attempted upon the reception of NAK using a random delay with a minimum value exceeding the coherence time of turbulence-induced fading channels. It was shown that the FSO systems using the CCHARQ protocol could maintain a significant performance improvement compared to that using the conventional ARQ one, especially in strong turbulence conditions.

In addition to the simple and cost-effective intensity modulation/direct detection (IM/DD) systems in [35], [125], the FSO coherent receivers are relatively more complex in the implementation, but they provide more flexibility and performance improvement [135]-[137]. By mixing the received signal with the strong local oscillator field, coherent receivers have better spatial and frequency selectivity than their non-coherent counterparts. Aiming to take advantage of the inherent features of coherent FSO systems, Aghajanzadeh et al. analyzed the performance of HARQ protocols, including TI, CC, and IR schemes, in terms of outage probability and throughput under the Gamma-Gamma statistical fading channel model [36]. Compared to the TI and CC schemes, IR-HARQ one was shown to be the most efficient throughput performance, thanks to the fact that only incremental redundancies are retransmitted to combine with the previously received ones for the correction of the corrupted frame.

It is noted that the studies mentioned above focussed on the performance evaluation of HARQ protocols under the impact of atmospheric turbulence without considering the pointing errors. In fact, using the similar approach as in [36], the analysis carried out therein for the performance of HARQ protocols is mathematically intractable in the presence of pointing errors. 
Table VIII: Literature review of HARQ protocols in different FSO system scenarios, including point-to-point terrestrial (PP), cooperative (CP), multi-hop relaying (MH), hybrid FSO/RF (HB), and satellite/aerial (SA) systems.

\begin{tabular}{|c|c|c|c|c|c|c|c|c|c|c|}
\hline \multirow{2}{*}{ Ref. } & \multirow{2}{*}{ Objective } & \multicolumn{3}{|c|}{ HARQ Type } & \multicolumn{5}{|c|}{ System } & \multirow{2}{*}{ Remarks } \\
\hline & & TI & $\mathbf{C C}$ & IR & $\mathbf{P P}$ & $\mathbf{C P}$ & MH & HB & SA & \\
\hline [119] & Throughput & & & $\bar{\nabla} \checkmark$ & $\bar{c} \checkmark$ & & & & & $\begin{array}{l}\text { Very first work studied the HARQ protocols in } \\
\text { the context of FSO communications }\end{array}$ \\
\hline [120] & Achievable Rate & & & $\checkmark$ & & & & $\checkmark$ & & $\begin{array}{l}\text { Propose an application of using rateless Fountain } \\
\text { codes for HARQ in hybrid FSO/RF systems }\end{array}$ \\
\hline$[35]$ & FER & & $\checkmark$ & & $\checkmark$ & & & & & $\begin{array}{l}\text { Highlight the substantial enhancement of FSO } \\
\text { systems using HARQ compared to pure ARQ }\end{array}$ \\
\hline [36] & $\begin{array}{l}\text { Outage Probability, } \\
\text { Throughput }\end{array}$ & $\checkmark$ & $\checkmark$ & $\checkmark$ & $\checkmark$ & & & & & $\begin{array}{l}\text { Confirm the effectiveness of FSO systems using } \\
\text { IR-HARQ over turbulence fading channels }\end{array}$ \\
\hline [121] & Throughput, FER & & & $\checkmark$ & & & & $\checkmark$ & & $\begin{array}{l}\text { Present a novel algorithm for HARQ using } \\
\text { different jointly optimized puncturing and RF bit } \\
\text { selection patterns for different retransmissions }\end{array}$ \\
\hline [122] & $\begin{array}{l}\text { Ergodic Capacity, Outage } \\
\text { Probability }\end{array}$ & & $\checkmark$ & $\checkmark$ & $\checkmark$ & & & & & $\begin{array}{l}\text { Evaluate the joint impact of turbulence and } \\
\text { pointing error on the performance of HARQ }\end{array}$ \\
\hline $\begin{array}{l}{[123],} \\
{[124]}\end{array}$ & $\begin{array}{l}\text { Throughput, Outage } \\
\text { Probability }\end{array}$ & & & $\checkmark$ & & & & $\checkmark$ & & $\begin{array}{l}\text { Evaluate the effect of adaptive power allocation } \\
\text { on hybrid FSO/RF systems using HARQ }\end{array}$ \\
\hline [125] & FER & & $\checkmark$ & & $\checkmark$ & & & & & $\begin{array}{l}\text { Evaluate the performance of HARQ in FSO } \\
\text { systems using Hermite-Gaussian beam patterns }\end{array}$ \\
\hline $\begin{array}{l}{[126],} \\
{[127]}\end{array}$ & Transmission Efficiency & $\checkmark$ & & & & & & & $\checkmark$ & $\begin{array}{l}\text { Investigate the impact of imperfect/delayed CSIs } \\
\text { on the performance of adaptive HARQ protocol }\end{array}$ \\
\hline [128] & $\begin{array}{l}\text { Ergodic Achievable Rate, } \\
\text { Outage Probability }\end{array}$ & & & $\checkmark$ & & & $\checkmark$ & & & $\begin{array}{l}\text { Study the design of HARQ protocols in the } \\
\text { context of multi-hop RF-FSO systems }\end{array}$ \\
\hline [129] & $\begin{array}{l}\text { Throughput, Delay, } \\
\text { Outage Probability }\end{array}$ & & $\checkmark$ & $\checkmark$ & $\checkmark$ & & & & & $\begin{array}{c}\text { Study the performance of HARQ in terms of } \\
\text { delay and packet sojourn }\end{array}$ \\
\hline [130] & $\begin{array}{l}\text { Throughput, BER, } \\
\text { Outage Probability }\end{array}$ & & $\checkmark$ & & & & & & $\checkmark$ & $\begin{array}{l}\text { The CC-HARQ using multi-frame interleaving is } \\
\text { an efficient solution for satellite FSO systems }\end{array}$ \\
\hline [131] & Average sum rate & & $\checkmark$ & $\checkmark$ & & $\checkmark$ & & & & $\begin{array}{l}\text { Propose a novel cooperative HARQ, which is } \\
\text { applicable for both CC and IR }\end{array}$ \\
\hline $\begin{array}{l}{[132],} \\
{[133]}\end{array}$ & $\begin{array}{l}\text { Throughput, Delay, } \\
\text { Energy Efficiency }\end{array}$ & & & $\checkmark$ & & & & & $\checkmark$ & $\begin{array}{l}\text { Propose a novel design of IR-HARQ based } \\
\text { sliding window mechanism for satellite systems }\end{array}$ \\
\hline [134] & Throughput, Delay & & & $\checkmark$ & & $\checkmark$ & & & $\checkmark$ & $\begin{array}{l}\text { A design proposal of cooperative IR-HARQ } \\
\text { protocols for satellite-HAP-vehicle FSO systems }\end{array}$ \\
\hline
\end{tabular}

From that fact, Zedini et al. investigated the performance of HARQ protocols over FSO turbulence channels, taking into consideration the impact of pointing misalignment [122]. Two widely deployed schemes, i.e., CC and IR, of HARQ protocols were analyzed from an information-theoretic perspective. It was shown that HARQ with IR outperforms CC-HARQ, especially for many HARQ transmission rounds. Using the same approach in [122], the delay performance of HARQ protocols, both CC and IR, was analyzed under the impact of combined atmospheric turbulence and pointing errors [129]. The IRHARQ protocol also achieved a better delay performance than CC-HARQ one in various turbulence strength and pointing jitter conditions.

2) Cooperative Systems: Recently, several studies on the design of HARQ protocols for cooperative FSO systems, either for terrestrial [131] or for space systems [134], have been reported. In particular, Hosseini et al. proposed a new cooperative HARQ (C-HARQ) protocol for broadcasting systems over FSO turbulence-induced fading channels [131]. The novel design applies to two well-known HARQ schemes, i.e., both CC and IR mechanisms, in the scenario of FSO broadcasting systems. In that scenario, the center node transmits the same information modulated by an optical beam to a finite number of fixed users. The SW mechanism was also applied for the operation of HARQ protocols.

Figure 9 illustrates a typical broadcasting FSO system using HARQ protocols with (1) conventional design and (2) novel cooperative design proposed in [131]. The broadcast inform- ation frames are transmitted via separate FSO links from a center node (S) to $N$ fixed users, i.e., $\mathrm{D}_{1}, \mathrm{D}_{2}, \cdots, \mathrm{D}_{N}$, with $N \geq 2$. The RF links are used for feedback communications, in which all feedback RF channels from the users to the center node are assumed to be error-free.

As a matter of fact, in the traditional HARQ systems, frame retransmissions are handled by the center node, and users only confirm the correct or erroneous nature of received frames via feedback channels. The users do not participate in the retransmission process, even though they could effectively cooperate in this process. Under the impact of atmospheric turbulence, the FSO systems can maintain better performance at shorter transmission distances. As a result, instead of relying on the center node, the frame retransmission process could be done by a neighboring user with a shorter distance than that from the center node and successfully decoded the original data frame. To this end, the authors [131] proposed a novel C-HARQ protocol for broadcasting FSO systems, in which selected neighbors would assist users in the retransmission process.

An example of C-HARQ protocol is depicted in Fig. 9, in which $\mathrm{D}_{1}$ and $\mathrm{D}_{2}$ are the neighboring users. Let $d_{\mathrm{SD}_{1}}$ and $d_{\mathrm{D}_{1} \mathrm{D}_{2}}$ denote the respectively transmission distances from $\mathrm{S}$ to $\mathrm{D}_{1}$ and $\mathrm{D}_{1}$ to $\mathrm{D}_{2}$, then $d_{\mathrm{SD}_{1}}>d_{\mathrm{D}_{1} \mathrm{D}_{2}}$. At the initial round, the $\mathrm{S}$ node broadcasts the frame $\mathrm{F}_{1}^{(1)}$ corresponding the subcodeword $C_{1}$ to all users, and then users return feedback signals, i.e., either ACK or NAK, to the S node. For the neighboring users, if the $\mathrm{D}_{2}$ node is unable to decode the 


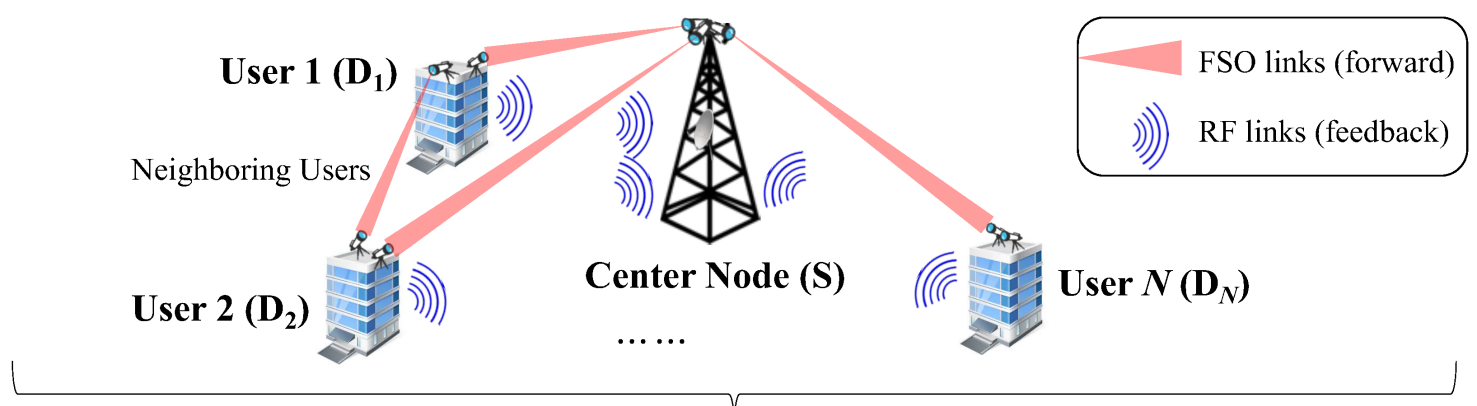

(1) Conventional approach
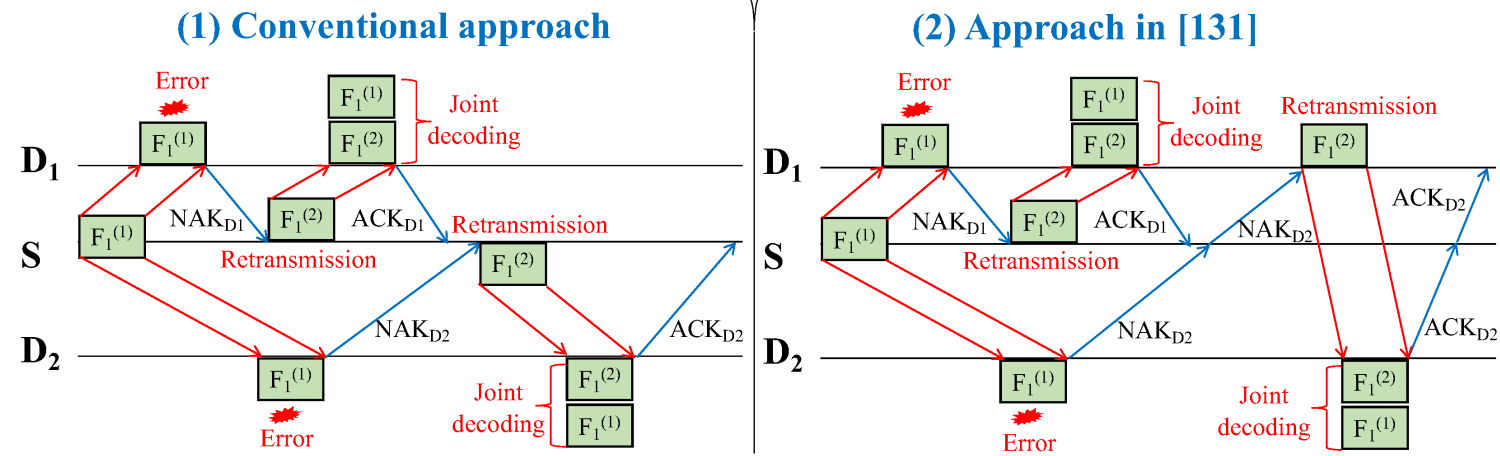

Figure 9: A typical broadcasting FSO system using HARQ protocols with (1) conventional approach and (2) novel cooperative approach in [131].

received frame $\mathrm{F}_{1}^{(1)}$, while the $\mathrm{D}_{1}$ node successfully decoded that frame after requesting a redundancy $\mathrm{F}_{1}^{(2)}$ from the $\mathrm{S}$ node. Then, the $S$ node informs $\mathrm{D}_{1}$ to encode data and retransmit the frame $\mathrm{F}_{1}^{(2)}$ to $\mathrm{D}_{2}$ for the next retransmission. At the $\mathrm{D}_{2}$, the newly received redundancy, i.e., $\mathrm{F}_{1}^{(2)}$, is combined with the previously received frame $\left(\mathrm{F}_{1}^{(1)}\right)$ to construct coded bits of code $C_{2}$ for decoding. The $\mathrm{D}_{1}$ node keeps retransmitting the redundancy to the $\mathrm{D}_{2}$ node until it is decoded successfully or reaches a predefined maximum number of transmission attempts of a frame, denoted by $M$. Here, the $\mathrm{D}_{2}$ node requests retransmissions by sending NAK signals to the $S$ node, and then the $S$ node informs the $\mathrm{D}_{1}$ node to retransmit frames to the $\mathrm{D}_{2}$ node. In addition, it is noted that each user is able to receive data frame from either the $\mathrm{S}$ node or only one neighboring user in each (re)transmission round.

It is worth noting that the C-HARQ based SW mechanism is not practically useful for FSO-based satellite communications due to the low achievable throughput. As a result, the sliding window mechanism, which allows higher efficiency by continuously transmitting frames without waiting for acknowledgment, would be an efficient solution for such longdistance and high-speed systems. The design of the C-HARQ based sliding window mechanism was addressed in HAP-aided relaying satellite FSO systems for the Internet of Vehicles (IoVs) [134]. In fact, the satellite-HAP-vehicle system consists of two links: (1) satellite-to-HAP and (2) HAP-to-vehicle links. As the altitude of HAP is in the stratosphere with less susceptibility to the weather effects, the pure sliding window ARQ mechanism was used for this link. Otherwise, the IRHARQ schemes were employed for the HAP-to-vehicle links, which experience weather-related issues like atmospheric turbulence and clouds. In such proposed HARQ protocols, the relay node, i.e., HAP, plays a vital role in retransmission of erroneous frames received by vehicles instead of requesting retransmission from the source, i.e., satellite. Moreover, using a pure sliding window ARQ mechanism for satellite-to-HAP links can avoid the complexity of decoding at HAP-based relaying nodes.

3) Multi-hop Relaying Systems: Multi-hop transmission is an alternative relay-assisted scheme, which employs relays in a serial configuration [138]-[140]. The scheme could mitigate the distance-dependent turbulence strength and atmospheric loss by utilizing shorter distances in resulting hops. The adoption of multi-hop relaying in FSO systems has been investigated for amplify-and-forward (AF) [140] and decodeand-forward (DF) [139] relaying techniques. Clearly, a large number of relays can considerably mitigate the impact of turbulence-induced fading; however, this is not a cost-effective solution. However, a report in [38] revealed the fact the multihop schemes often fail to provide reliability in turbulence conditions, as they will be forwarding the noisy replicas of the received data.

On the other hand, in the RF domain, mmWave has emerged as the enabler to achieve a sufficiently high data rate, comparable to that in the FSO links [141]. From this perspective, the combination of FSO and mmWave-based RF links is considered as a powerful candidate for high data-rate multihop communications [142]. To maintain reliable transmissions, Makki et al. addressed the design of IR-HARQ protocol for such multi-hop RF-FSO systems operating in the DF mode [128]. In addition, the performance of IR-HARQ protocols was analyzed from an information-theoretic point of view.

Regarding the HARQ protocol operation in the DF mode, the successfully received data frame at each hop is decoded and re-encoded for the transmission to a next-hop. Otherwise, 
only sub-codewords divided from a parent codeword (i.e., low code rate) are retransmitted and combined with all previously received sub-codewords to decode that frame. Here, the SW mechanism was used in IR-HARQ protocols, which processes one frame at a time. At each hop, the retransmission continues until the frame is correctly decoded or the maximum permitted transmission round is reached. In the latter case, the frame is clarified to be lost. Therefore, a frame is successfully received by the destination if it is correctly decoded in all hops. With typical parameter settings of the RF-FSO dual-hop systems, i.e., the outage probability of $10^{-4}$ and code rate of 3 natsper-channel-use, the HARQ protocols with the persistent level of 2 and 3 reduce the required powers, compared to cases without HARQ, by 13 and $17 \mathrm{~dB}$, respectively.

4) Hybrid FSO/RF Systems: The state-of-art hybrid FSO/RF systems using HARQ protocols were based on the parallel/simultaneous FSO/RF links [120], [121], [123], [124]. In these studies, for the sake of simplicity in the analysis, the SW mechanism, handling one frame at a time, was used for the operation of HARQ protocols. In addition, realizing the potential of incremental redundancy schemes, most of the works focussed on the IR-HARQ protocols, which offer the highest efficiency compared to other HARQ types. As a matter of fact, the IR-HARQ protocol is based on the puncturing technique, which allows an encoder/decoder pair to adjust code rates without changing their structure [143]. Variablerate codes can be obtained by deleting some coded bits from a low-rate encoder (called mother code), according to a specific punctured pattern [144].

Several studies presented the novel punctured structures for the operation of IR-HARQ protocol to improve the system reliability in hybrid FSO/RF communications. Specifically, AbdulHussein et al. addressed the design of IR-HARQ protocols, using rateless Fountain codes [145], for such hybrid $\mathrm{FSO} / \mathrm{RF}$ systems in [120]. This design involves the use of Raptor codes as described in [146], in which the data is partitioned into two sets of bits, separately encoded, and sent through FSO/RF channels. The advantages of such a HARQ protocol design are twofold. Firstly, it is an elegant approach for data transmissions over the time-varying behavior of $\mathrm{FSO} / \mathrm{RF}$ channels, as it requires neither a bank of codes with various rates nor explicit code selection. As a result, other considerations aside, one may choose this design simply due to its ease of operation. Secondly, no rate mismatch due to outdated or inaccurate channel estimation can happen. This is a distinct performance benefit over schemes with code-rate selection, e.g., [126], [127], which may fail if the channel quality varies notably from one codeword to another. In fact, as for hybrid FSO/RF systems, these variations may happen primarily due to temporal fluctuations in received optical powers caused by atmospheric turbulence. Moreover, the authors also established the pertinent information-theoretic limits showing that coding schemes with code-rate selection may suffer from rate loss or outages depending on channel conditions. It was demonstrated that HARQ protocol, based on a rateless coding scheme, using an off-the-shelf Raptor code [147], well approaches the information-theoretic limits regardless of channel conditions.
Another design approach for IR-HARQ protocols in hybrid FSO/RF systems was mentioned in [121]. This study addressed a novel IR-HARQ algorithm with a joint puncturing pattern and $\mathrm{FSO} / \mathrm{RF}$ bit selection optimized for hybrid FSO/RF fading channels. In contrast to the rate-compatible punctured codes for conventional IR-HARQ protocols, this design also involves bit splitting between FSO and RF in each retransmission, affecting the puncturing pattern selection. The algorithm uses separate puncturing and RF bit selection patterns in each retransmission based on minimizing the BER. The puncturing patterns for several ECC codes, including nonsystematic/systematic convolutional codes and turbo code, are considered.

Differently from [120] and [121], the authors analyzed the performance of IR-HARQ protocols based on the conventional punctured structure, considering the joint data transmission/reception in the FSO and RF links [123], [124]. The data sequence is encoded into parallel FSO and RF bit streams, which are then simultaneously sent to the receiver side for joint decoding. Moreover, the adaptive power allocation was exploited to improve the performance of hybrid FSO/RF systems using HARQ protocols. Due to the complexity of adaptive power allocation, it was recommended to use uniform power allocation for HARQ-based RF-FSO links at high received SNR values.

5) Satellite/Aerial Systems: In long-distance FSO systems, the HARQ protocol is expected to be a promising link-layer error-control solution over time-varying turbulence fading channels, which circumvents the limitations of other solutions, i.e., pure ARQ protocols and ECC schemes. The main reason for such limitations is that the ECC scheme often requires excessive redundancies to guarantee the transmission reliability and hence results in inefficient throughput performance [148]. Moreover, ARQ protocols often fail to provide satisfactory delay and throughput performance for long-distance and highlatency systems, as they require many retransmissions for erroneous frames [70].

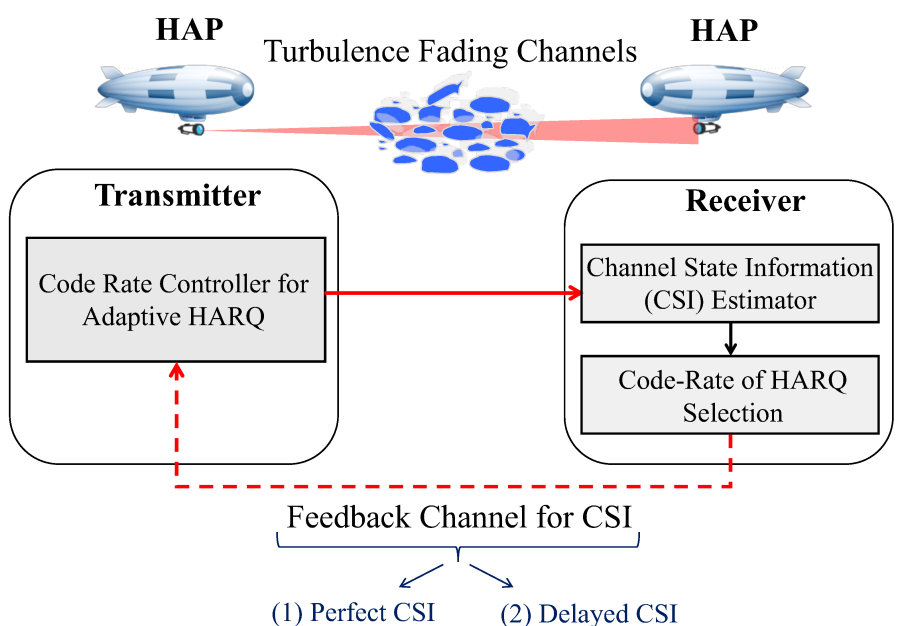

Figure 10: Adaptive TI-HARQ protocol with code-rate selected based on the FSO feedback channel for (1) perfect CSI and (2) delayed CSI. 
Table IX: Coding gain $(\mathrm{dB})$ for adaptive HARQ with different CSI qualities compared with pure SR-ARQ [126].

\begin{tabular}{|c|c||c|c||c|c|}
\hline \multirow{2}{*}{$\begin{array}{c}\text { Distance } \\
(\mathrm{km})\end{array}$} & $\begin{array}{c}\text { Coherence } \\
\text { Time } \\
(\mathrm{ms})\end{array}$ & \multicolumn{2}{|c||}{ Gain for TE $=30 \%$} & \multicolumn{2}{c|}{ Gain for TE $=90 \%$} \\
\cline { 3 - 6 } & & $\begin{array}{c}\text { Delayed } \\
\text { CSI }\end{array}$ & $\begin{array}{c}\text { Perfect } \\
\text { CSI }\end{array}$ & $\begin{array}{c}\text { Delayed } \\
\text { CSI }\end{array}$ & $\begin{array}{c}\text { Perfect } \\
\text { CSI }\end{array}$ \\
\hline \hline \multirow{2}{*}{300} & 10.95 & 3.6 & 4.8 & 2.6 & 3.4 \\
\cline { 2 - 6 } & 2.7 & 2.9 & 4.5 & 0.7 & 3.3 \\
\hline \multirow{2}{*}{600} & 13.75 & 3.2 & 4.4 & 2.4 & 5.4 \\
\cline { 2 - 6 } & 2.75 & 1.9 & 4.1 & 0.3 & 3.6 \\
\hline
\end{tabular}

As a result, many studies on the design of HARQ protocols have been recently reported for satellite/aerial FSO systems using TI-HARQ protocol [126], [127], CC-HARQ protocol [130], and IR-HARQ protocol [132], [133]. Notably, Parthasarathy et al. introduced a design of adaptive TI-HARQ protocols based on the combination of ECC scheme using Reed-Solomon (RS) codes and SR-ARQ for retransmission mechanism in inter-HAP FSO communication systems [126], [127]. For the operation of adaptive TI-HARQ protocols, the coding rate of RS code varies accordingly to the channel conditions. In other words, the proper code rates are selected based on the feedback signals from the receiver, i.e., channel state information (CSI), which is illustrated in Fig. 10. In case of a frame transmission failure indicated by an ARQ timeout, the corresponding frame is retransmitted using the current coding rate based on the CSI at the transmitter. Indeed, the CSI at the transmitter plays an essential role in adapting the coding rate of TI-HARQ operation, thereby achieving maximum throughput and reducing the delay due to retransmissions. It is noted that the estimated CSIs in practical long-distance FSO systems are delayed due to the channel propagation time. This kind of delay is in the order of milliseconds, which is similar to the channel coherence time. Therefore, it is crucial to understand and investigate the impact of such delayed CSIs on the performance of adaptive TI-HARQ protocols in longdistance inter-HAPs FSO systems. The transmission efficiency (TE), defined as the fraction of the channel bit rate usable for payload transport, was analyzed in the presence of perfect and delayed CSIs at the transmitter [126], [127]. Table IX [126] illustrates the coding gain for the adaptive TI-HARQ protocols with different CSI qualities compared to the pure SR-ARQ protocols in the maintenance of TE of $30 \%$ and $90 \%$. From Table IX [126], we observe a considerable gain improvement for the adaptive TI-HARQ protocols in comparison with the uncoded non-adaptive ARQ protocol only implementation for the scenarios of FSO-based inter-HAPs communications.

It is worth noting that FSO-based inter-HAP systems are located at altitudes of $17-25 \mathrm{~km}$, where the impact weather is negligible [149]. Therefore, using the simplest TI variant for the HARQ protocol is sufficiently reliable in such systems. Nevertheless, for FSO-based satellite-to-ground systems, more efficient HARQ schemes should be employed considering the impact of various factors, such as atmospheric turbulence, cloud coverage, atmospheric attenuation, and pointing misalignment. In [130], Xiang et al. proposed an improved HARQ protocol combining the multi-frame interleaving and
CC mechanism for satellite-to-ground FSO communication systems. In fact, the traditional CC-HARQ protocol generally uses interleaving schemes to disperse the serial burst errors and then employs an ECC scheme for the error correction. This conventional protocol is, however, often fails to provide the system reliability in strong turbulence conditions, as the error codewords are evenly distributed in the error correction part after interleaving. By using the multi-frame interleaving scheme, improved HARQ protocol can offer better performance than the traditional CC-HARQ one in strong conditions of turbulence-induced fading channels.

Compared to the CC-HARQ protocol, IR-HARQ offers better throughput performance as it only retransmits incremental redundancies [122]. In addition, the sliding window protocols were confirmed to be the efficient solution for FSO-based satellite systems (refer to Section II. 4). From the issues mentioned above, a novel design of IR-HARQ based sliding window mechanism was recently addressed in FSO-based satellite communication systems [132], [133]. Furthermore, its integration with PHY adaptive multi-rate scheme was considered to further enhance the system performance [133]. For the operation of the IR-HARQ protocol, the rate-compatible punctured convolutional (RCPC) code family with Viterbi decoding is used to implement the successive incremental redundancy transmissions for the frame error correction. Additionally, the operation of the IR-HARQ protocol is applied to each burst containing multiple frames. To effectively facilitate the operation of IR-HARQ with adaptive multi-rate burst transmissions, the HARQ's window size is designed by a burst size. Essential performance metrics for satellite systems, including average throughput, average frame delay, and energy efficiency, were addressed for the IR-HARQ protocol. As is expected, it was shown the significant performance improvement of IR-HARQ based sliding window mechanism compared to existing state-of-art on ARQ solutions mentioned earlier in Section II for long-distance satellite-based FSO communications. In addition, the tradeoff between energy efficiency and throughput was also explored, in which it was not possible to jointly optimize between them.

\section{Summary and Future Work}

This section reviewed the existing studies on the design and performance estimation of HARQ protocols in different FSO system scenarios. The remarkable points of this section can be summarized as follows.

- The state-of-art on HARQ protocols revealed that IRHARQ is the most efficient variant for different FSO system scenarios, thanks to retransmissions of only redundancy codewords for jointly decoding with the previously received ones.

- Aside from [132]-[134], most of the studies addressed the use of SW mechanism for the operation of HARQ protocols for the sake of simplicity in the analysis.

- A lot of studies on the HARQ protocol focussed on analyzing its performance over turbulence fading channels from an information-theoretic approach, e.g., [35], [36], [122], [128], [131]. 
- Using rateless codes [120], modified punctured structure [121], and adaptive power allocations [124] are existing solutions to enhance the performance of IR-HARQ protocols in the context of hybrid FSO/RF systems.

- A joint design of adaptive transmissions, either adaptive coding schemes [126], [127] or adaptive rate transmissions [133], with HARQ protocols can offer a significant performance improvement in FSO-based satellite/aerial communication systems. In addition, the issues of imperfect estimated CSIs on the joint design between adaptive schemes and HARQ protocols using the simplest TI variant were addressed in [126], [127].

- The summary of existing works on HARQ protocols in different FSO communication systems is given in Table VIII.

In addition, some potential research topics on HARQ protocols can be further investigated, for example:

- The HARQ protocols can be potentially considered for the emerging scenarios of FSO-based satellite/aerialassisted IoVs to maintain reliable connectivity. The strict delay requirement is essential for such network scenarios, while the current HARQ design's primary focus is on throughput performance. As a matter of fact, HARQ is commonly considered a high-latency scheme. Such latency comes from multiple retransmissions, multiple message decoding at the receiver, and feedback signals (ACK/NAK) transmission/processing delay. Therefore, to deploy HARQ protocols in such IoVs network scenarios, it is necessary to minimize the transmission delay of the HARQ protocols. One of the possible solutions is using HARQ without waiting for feedback based on channel conditions, which has been considered in different work/standard specifications in the domain of RF-based communications, e.g., [150]-[152]. The promising results obtained in the most recent study in [152] confirmed the effectiveness of using fast HARQ protocols to minimize the end-to-end delay for RF systems. Considering such HARQ protocol design in the context of FSO-based satellite/aerial-assisted IoVs networks would be exciting and a possible direction for future study.

- Machine learning techniques have effectively advanced the state-of-the-art for many research problems in wireless communication networks [153]. To enhance the performance of systems using HARQ protocols, which imposes additional delay and energy consumption due to retransmissions required, machine learning techniques have been recently investigated in [154]. This allows providing the HARQ feedback earlier by predicting the decoding outcome, enabling the original transmitter to react faster to the current channel conditions and send additional redundancy at an earlier point. In this way, more HARQ iterations are encouraged to improve system reliability under strict delay constraints. For future work, this approach could be potentially considered to enhance the systems using HARQ protocols in FSO communications.

\section{Cross-Layer Design Approach}

Cross-layer design, which allows information sharing among layers, has been widely investigated in RF-based wireless networks. Thanks to the promising results achieved, this approach has recently attracted research efforts in the domain of FSO networks. Indeed, realizing cross-layer design is essential for improving the performance of FSO systems over atmospheric turbulence channels. This section addresses the cross-layer design approach for considered linklayer retransmission-based error-control, including ARQ and HARQ, protocols in FSO communications. To this purpose, we first review the background of design methodologies for such protocols, including the classification, motivation, and challenging issues. Next, we introduce several crosslayer design frameworks of link-layer retransmission protocols incorporating protocols at the PHY and transport layers in FSO networks. Finally, we summarize key issues and present potential research topics related to this design approach.

\section{A. Overview of Design Methodologies}

Design methodologies: For designing the link-layer retransmission-based error-control protocols in FSO communications, two methodologies have been investigated so far, i.e., (1) layered design and (2) cross-layer design. In the following, we briefly present the concept of these methodologies.

- Layered design: The protocols on one layer are designed independently from the protocols on other layers. In this design, the communication among non-adjacent layers is not permitted [155]. Although the strict boundary between the layers makes the networks easy to deploy, the layers' encapsulation prevents some necessary information sharing between the layers. As a result, this design approach does not provide a mechanism for the performance optimization between different protocol layers, which can significantly improve the system performance.

- Cross-layer design: Unlike the layered design, the crosslayer approach allows the interaction between layers by permitting one layer to access the data of another layer for the joint optimization of protocols across the communication stack [156]. In fact, the cross-layer design provides inter-layer communication between non-adjacent layers without destroying the existing network reference models. In other words, the cross-layer design implies that each layer can share parameters, status, and additional information with the different layers without breaking the layers structure of computer networks. Indeed, the networks employing the cross-layer design can provide a better performance, in which many studies have been confirmed the poor performance of layered design over the cross-layer one in wireless networks, e.g., [157][160].

Cross-layer design for FSO networks: The motivation of the cross-layer design originates from the unique and novel characteristics of FSO networks. As a matter of fact, the traditional layered architecture reference models, including the open systems interconnection (OSI) and Transmission Control Protocol/Internet Protocol (TCP/IP), were initially 
designed for wired networks [161]. Nevertheless, as the fading channel models in FSO networks are entirely different from that of wired networks, the problems created by the FSO networks but solved with the layered architecture may result in unsatisfactory results and poorly achievable performance. Additionally, an individual TCP/IP protocol usually aims to solve one specific set of problems without considering the end-to-end network performance leading to the deployment of these protocols does not always satisfy the increased performance requirements [155]. Furthermore, the time-varying turbulence fading channels and new issues in FSO networks motivate the consideration of cross-layer design, which can address these characterizations by exploiting the interactions and dependencies among layers. For example, the performance of TCPs over FSO networks mainly depends on the congestion loss caused by the network's buffer overflows and transmission errors due to the uncertainty of atmospheric FSO last-mile channels [162]. Instead of executing the TCP's congestion control in isolation at the transport layer, by using the crosslayer design approach, the congestion control can be jointly managed with buffer management at the network layer, the error-control methods at the link layer and rate adaptation transmission at the physical layer [163]. As a result, the performance of TCP could be improved significantly.

Challenging issues of cross-layer design: Cross-layer designs offer a significant number of benefits, e.g., joint optimization of protocols from multiple layers to increase the system throughput, reduce latency, and minimize the transmission error rate [155]. Nevertheless, there are several drawbacks/challenges of cross-layer designs that are inevitable due to the characteristics of these designs, as follows [158]:

- Cross-layer overhead/signaling: It is inevitable to result in an extra overhead and control signaling when exchanging the cross-layer information in networks [164]. A large amount of such information may occupy a great deal of bandwidth, leading to a burden on the network performance.

- Coexistence of multiple cross-layer designs: It is not straightforward to integrate different cross-layer designs into a uniform design due to the specific communication standard of each cross-layer design [161]. As a result, the coexistence problem is a challenge that cross-layer designs have to deal with.

- Universal cross-layer design: The cross-layer model for a single application may not be suitable for different applications. For instance, video streaming requires a small ARQ persistent level, while the data transmission can endure a high ARQ persistent level in the errorprone environment of wireless networks [165]. Finding a universal cross-layer standard for different applications poses various challenges and is an open research issue.

- Destruction of layered architecture: Cross-layer frameworks may break the encapsulation of layers in the conventional layered architecture. As a matter of fact, a slight modification in one layer results in a series of changes in the other layers [166]. Therefore, the destruction of layered architecture is one of the most challenging issues and the fundamental drawbacks of the cross-layer design.

\section{B. Cross-Layer Frameworks in FSO Networks}

In the domain of FSO communications, there are several studies on the link-layer retransmission-based error-control protocols, including both ARQ and HARQ, regarding the cross-layer designs [33], [34], [133], [162], [167], [169], [170]. As shown in Fig. 11, these works are mainly partitioned into two groups, i.e., the cross-layer designs between (i) physical layer and link layer (PHY/Link design) [33], [34], [133] and (ii) link layer and transport layer (Link/Transport design) [162], [167], [169], [170]. A summary of the literature concerning the cross-layer designs of link-layer ARQ and HARQ protocols in FSO networks is provided in Table X. In the following, we present the details of each cross-layer design group.

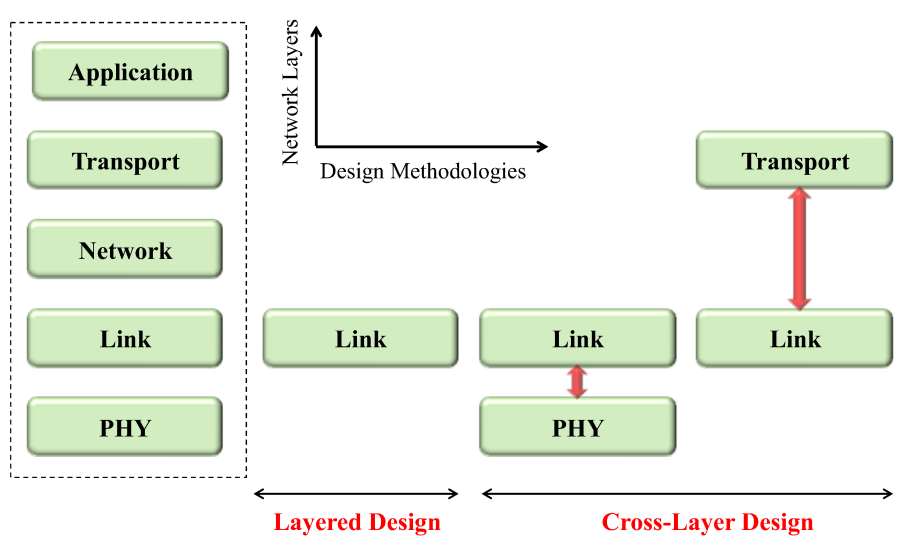

Figure 11: Existing design methodologies of link-layer retransmission-based error control protocols in FSO networks.

1) PHY/Link Cross-layer Design: Atmospheric turbulence, which results in a very slowly-varying fading, is a major degrading factor in FSO systems. As reported in [172], [173], the channel coherence time is typically 1 to $10 \mathrm{~ms}$ or longer, and a Gbps-transmission-rate period may cover up to thousands of consecutive frames. This quasi-static channel property makes providing reliable feedback possible, and the available CSI at the transmitter can be used to design the adaptive transmission schemes for considerable performance enhancement in FSO systems. Moreover, the feedback information required in the adaptive transmission is relatively feasible to implement in FSO systems. The reason is that commercially available FSO units have full-duplex (bi-directional) capabilities, and a small portion of the large available bandwidth could be allocated for feedback purposes without much effect on the data rate [14]. As a result, adaptive transmission, one of the most popular error-control PHY schemes, emerges as a promising solution for FSO systems. Adaptive transmission has been extensively studied in the context of FSO communications [174]-[177] and involves the change of system parameter settings, e.g., transmission rate, coding rate, modulation order, transmitted power, or the combination of those according to the channel conditions. In this regard, the cross-layer design between linklayer retransmission protocols and adaptive transmissions, an 
Table X: Summary of the literature considering cross-layer designs of link-layer ARQ/HARQ protocols in FSO networks.

\begin{tabular}{|c|c|c|}
\hline Cross-Layer Design & Frameworks & Results \\
\hline PHY/Link design & $\begin{array}{l}\text { - Joint design of AR transmissions with (i) conventional } \\
S W-A R Q \text { and (ii) proposed FC-SW-ARQ [33] } \\
\text { - Cross-layer design between sliding window ARQ and } \\
\text { rate adaptation burst transmission [34] } \\
\text { - Design of IR-HARQ based sliding window mechanism } \\
\text { with AR satellite burst transmission [133] }\end{array}$ & $\begin{array}{l}\text { - The FSO systems employing cross-layer designs } \\
\text { achieve substantially better spectral efficiency and trans- } \\
\text { mission reliability than conventional systems with layered } \\
\text { designs. Among cross-layer designs, AR/FC-ARQ per- } \\
\text { forms more efficiently than AR/ARQ. } \\
\text { - The sliding window protocols, i.e., both GBN and SR } \\
\text { schemes, outperform SW-ARQ one in terms of through- } \\
\text { put performance. Moreover, SR-ARQ always provides } \\
\text { better performance, including throughput, delay and } \\
\text { frame loss rate, than GBN-ARQ, especially in strong } \\
\text { turbulence conditions. } \\
\text { - The considerable performance improvement of the } \\
\text { proposed IR-HARQ based sliding window mechanism } \\
\text { compared to the other solutions, i.e., IR-HARQ based SW } \\
\text { one and pure sliding window ARQ, over FSO satellite-to- } \\
\text { ground turbulence channels is highlighted. In addition, it } \\
\text { is not possible to jointly maximize the energy efficiency } \\
\text { and throughput, where the tradeoff between them is } \\
\text { explored. }\end{array}$ \\
\hline Link/Transport design & $\begin{array}{l}\text { - The use of SR-ARQ to improve the TCP throughput } \\
\text { performance [167] } \\
\text { - Joint design of AR transmissions and SR-ARQ to } \\
\text { enhance the throughput performance of TCPs [162] } \\
\text { - Simulation framework considering PHY error models, } \\
\text { SR-ARQ protocol, and TCP variants [168] } \\
\text { - Cross-layer framework incorporating the IR-HARQ } \\
\text { and TCP variants for satellite-assisted vehicular networks } \\
\text { [169], [170] } \\
\text { - Cross-layer framework incorporating the PHY FEC } \\
\text { code, link-layer SR-ARQ, and TCP variants for satellite- } \\
\text { based hybrid FSO/RF vehicular networks [171] }\end{array}$ & $\begin{array}{l}\text { - Using the SR-ARQ could considerably reduce the } \\
\text { transmitted power required to maintain a maximum TCP } \\
\text { throughput level. Additionally, it is recommended that the } \\
\text { optimal value of the SR-ARQ persistent level is } 6 \text { for the } \\
\text { considered networks. } \\
\text { - The TCP performance could be controlled by the } \\
\text { persistent level of SR-ARQ protocol and the targeted FER } \\
\text { of AR transmissions, in which the optimal value of TCP } \\
\text { throughput is explored. } \\
\text { - Retransmissions at the link-layer significantly reduce } \\
\text { the TCP segment-loss probability, in which the maximum } \\
\text { window size can be achieved over time at a high persist- } \\
\text { ent level of ARQ protocols. } \\
\text { - The effectiveness of using IR-HARQ based sliding } \\
\text { window mechanism in improving the TCP throughput } \\
\text { performance over the FSO last-mile access of satellite- } \\
\text { assisted vehicular networks is confirmed. } \\
\text { - The joint of PHY FEC code and link-layer ARQ can } \\
\text { effectively improve the TCP throughput performance in } \\
\text { the context of hybrid FSO/RF networks. }\end{array}$ \\
\hline
\end{tabular}

exciting but challenging research problem, would effectively improve the overall system performance over turbulenceinduced fading channels.

The joint design of AR transmissions and ARQ protocols in FSO terrestrial systems was studied in [33], [34]. The design frameworks incorporating AR scheme and different ARQ mechanisms investigated in [33], [34] are shown in Fig. 12. In these works, the objective of AR transmission is to maximize the data rate over the turbulence fading channels while satisfying a required QoS at the link layer, i.e., targeted FLR [33] (denoted as FLR target) and targeted FER [34] (denoted by FER $_{\text {target }}$ ). Using the assumption of perfect estimated CSIs, multiple transmission modes with different data rates can be adjusted dynamically at the transmitter. For the design of AR transmissions, the entirely received SNR range is partitioned into $K+1$ non-overlapping consecutive intervals with boundary points denoted as $\left\{\gamma_{k}\right\}_{k=0}^{K}$. The channel is said to be in the state $k$-th if the received SNR falls into the interval of $\left[\gamma_{k-1}, \gamma_{k}\right)$, where $k \in\{0,1,2, \cdots, K\}$. Then, each channel-state is assigned by a specific transmission mode, which can bring the highest possible data rate while maintaining the targeted $\mathrm{FER}_{\text {target }}$. More particularly, the cross-layer design framework between AR scheme and truncated SW-ARQ protocol, which processes one frame at a time, was introduced in [33]. Two cross-layer designs, i.e., (i) $\mathrm{AR}$ and standard-ARQ (AR/ARQ) and (ii) AR and ARQ with FC (AR/ARQ-FC), were investigated. In this study, the frame duration of ARQ operation was adjusted based on the transmission modes of the AR scheme as depicted in the approach (1) in Fig. 12. It is noted that the number of available AR scheme's modes, denoted by $N$, is supposed to be equal to the number of channel states, i.e., $N=K$. As a result, the selection of SNR thresholds satisfies the condition that the FLR for each channel-state is exactly the predefined FLR target. Using the truncated SW-ARQ with $N_{\mathrm{t}}$ maximum transmission attempt and $M$-QAM modulation for AR transmissions, the design problem can be formally formulated as [33]

$$
\mathrm{FLR}_{n} \doteq \mathrm{FLR}_{\text {target }}= \begin{cases}\mathrm{FER}_{n}(M, \gamma)^{N_{\mathrm{t}}}, & \text { for AR/ARQ }, \\ \prod_{l=1}^{N_{\mathrm{t}}} \operatorname{FER}_{n, l}(M, \gamma), & \text { for AR/ARQ-FC, }\end{cases}
$$

where $M=2^{n}$ with $n \in\{1,2, \cdots, N\}$, and $\operatorname{FER}_{n, l}(M, \gamma)$ is the instantaneous FER when using mode $n$ on the $l$-th transmission attempt. Given the equation of $\operatorname{FER}_{n}(M, \gamma)$ in $[33,(10)]$, the 


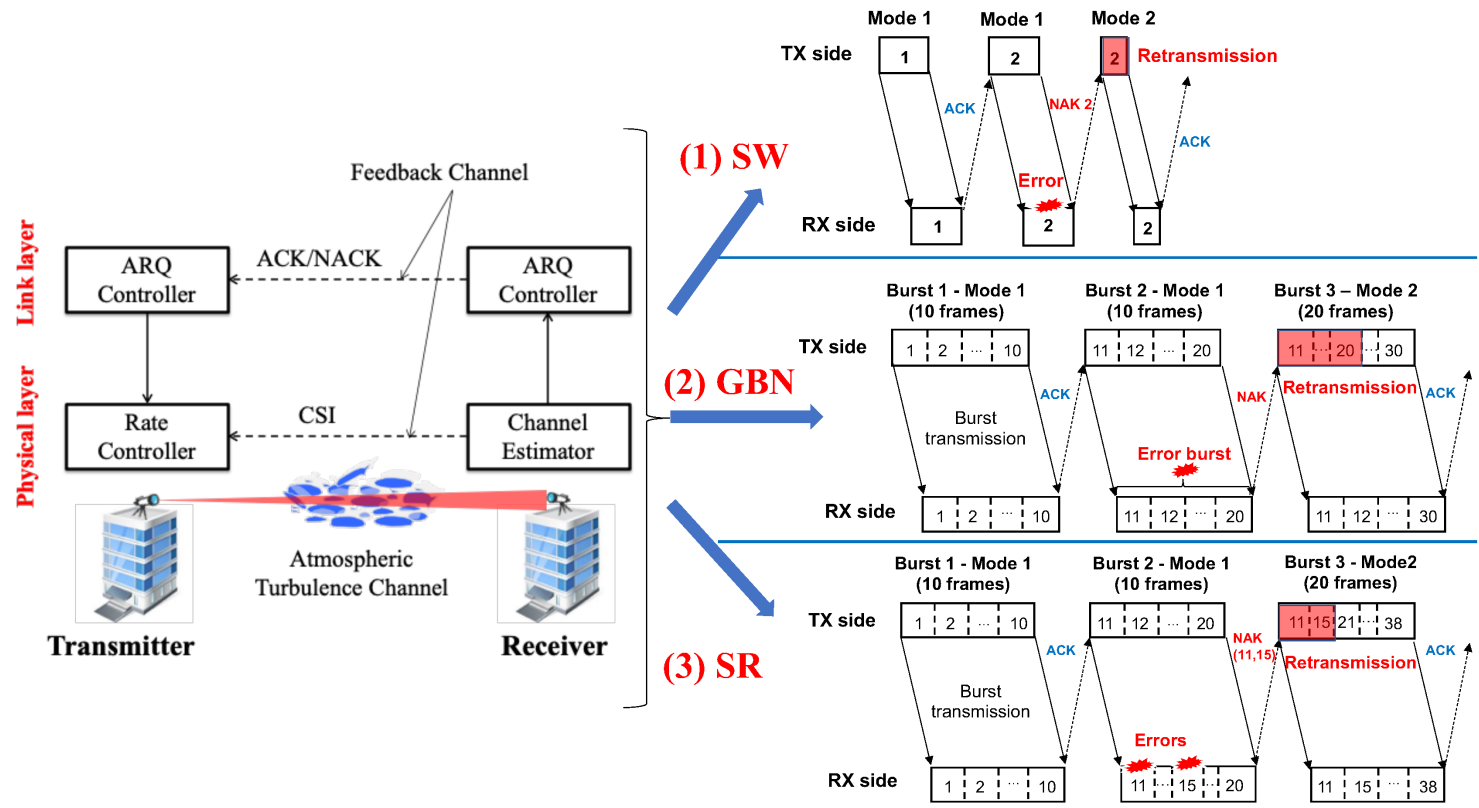

Figure 12: Existing design frameworks incorporating AR scheme and different ARQ mechanisms, i.e., (1) SW [33], (2) GBN [34], and (3) SR [34] for FSO terrestrial systems.

SNR thresholds can be obtained as [33]

$$
\gamma_{n}= \begin{cases}-\frac{2\left(2^{n}-1\right)}{3} \ln \left(\frac{5 \mathrm{FLR}_{\text {target }}^{\frac{1}{N_{\mathrm{t}}}}}{N_{\mathrm{f}}}\right), & \text { for AR/ARQ, } \\ -\frac{4\left(2^{n}-1\right)}{3\left(N_{\mathrm{t}}\right)\left(N_{\mathrm{t}}+1\right)} \ln \left(\frac{\mathrm{FLR} \text { target }}{\left(0.2 N_{\mathrm{f}}\right)^{\mathrm{t}_{\mathrm{t}}}}\right), & \text { for AR/ARQ-FC, }\end{cases}
$$

where $N_{\mathrm{f}}$ is the frame size.

Differently from [33], the sliding window ARQ, which allows the transmission of multiple frames simultaneously, was jointly designed with the AR scheme in [34]. Both sliding window protocols, i.e., GBN and SR, were investigated. Also, their integration with AR transmission is illustrated in Fig. 12, in which the approaches (2) and (3) are for AR/ARQ-GBN and AR/ARQ-SR, respectively. To effectively facilitate sliding window protocols with the AR scheme under the impact of atmospheric turbulence, the ARQ's window size, which is based on the burst transmission, was designed considering the time-varying behavior of the turbulence-induced fading channels. In particular, the data are transmitted in fixed-time bursts, in which each channel-state covers a burst transmission. Then, the selection of SNR thresholds satisfies the condition that intervals of all channel states, which are shorter than the fading channel coherence time $\left(t_{\mathrm{c}}\right)$, are equal to the burst duration $\left(T_{\text {burst }}\right)$. It is critical to note that channel thresholds $\left\{\gamma_{k}\right\}_{k=0}^{K}$ are not the same as $\gamma_{n}^{*}, n \in\{1,2, \cdots, N\}$, which are used for the selection of transmission modes obtained by a least-square curve fitting of FER in [178]. To determine the threshold levels of channel states, the first channel-state $\left[\gamma_{0}, \gamma_{1}\right)$ was set to the outage state, i.e., equivalent to $\left[-\infty, \gamma_{1}^{*}\right)$, and the average channel-state duration $\bar{\tau}_{k}$ defined in [34, (9)] was set to $T_{\text {burst }}$. The method to search for thresholds $\left\{\gamma_{k}\right\}_{k=0}^{K}$ is summarized in Algorithm 1.

The channel-state $k$-th with the SNR interval of $\left[\gamma_{k-1}, \gamma_{k}\right)$ is said to be in the transmission mode $n$ if it brings

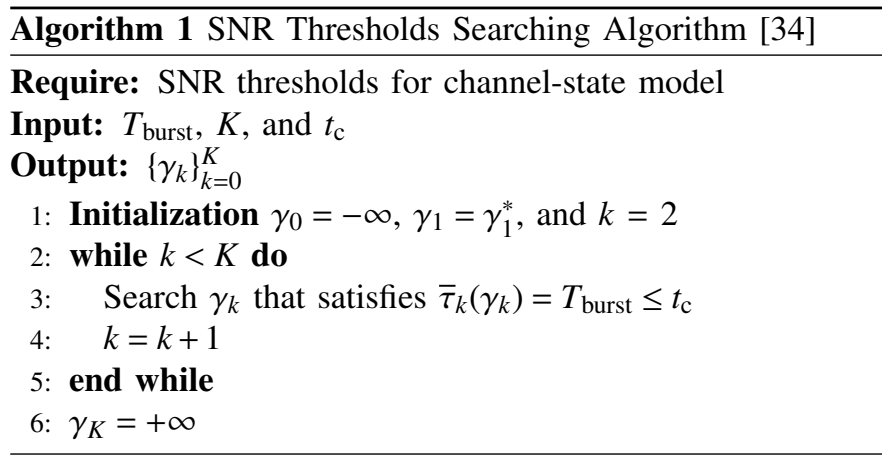

the highest possible data rate, and the corresponding av-

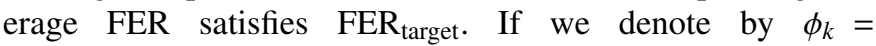
$\{n \mid k-$ th state is assigned by mode $n\}$, the design problem can be formally formulated as

$$
\begin{array}{ll}
\underset{\mathbf{n}}{\operatorname{maximize}} & R_{\phi_{k}}(\mathbf{n}) \\
\text { subject to } & \overline{\operatorname{FER}}_{k}\left(M_{\phi_{k}}, \gamma\right) \leq \mathrm{FER}_{\text {target }}, \\
& M_{\phi_{k}}=2^{n}, \quad n=1,2, \cdots, N,
\end{array}
$$

where $R_{\phi_{k}}(n)$ and $\overline{\operatorname{FER}}_{k}\left(M_{\phi_{k}}, \gamma\right)$ are the data rate and average FER at the state $k$-th using mode $n$, respectively. Here, $R_{\phi_{k}}(n)=$ $R_{s} \log _{2} M_{\phi_{k}}$ with $M_{\phi_{k}}=2^{n}$ and $R_{s}$ the symbol rate.

The throughput comparison among cross-layer designs for FSO terrestrial systems, including AR/ARQ-SW, AR/ARQGBN, and AR/ARQ-SR, are shown in Fig. 13. As is evident, the AR/ARQ-based sliding window mechanisms outperform AR/ARQ-SW thanks to the transmission of many frames without waiting for an acknowledgment. The essential point is that the throughput improvement becomes much more considerable when the symbol rates are high. The reason is that the time waiting for the acknowledgment of a data frame (twice the propagation delay) in AR/ARQ-SW becomes significant 


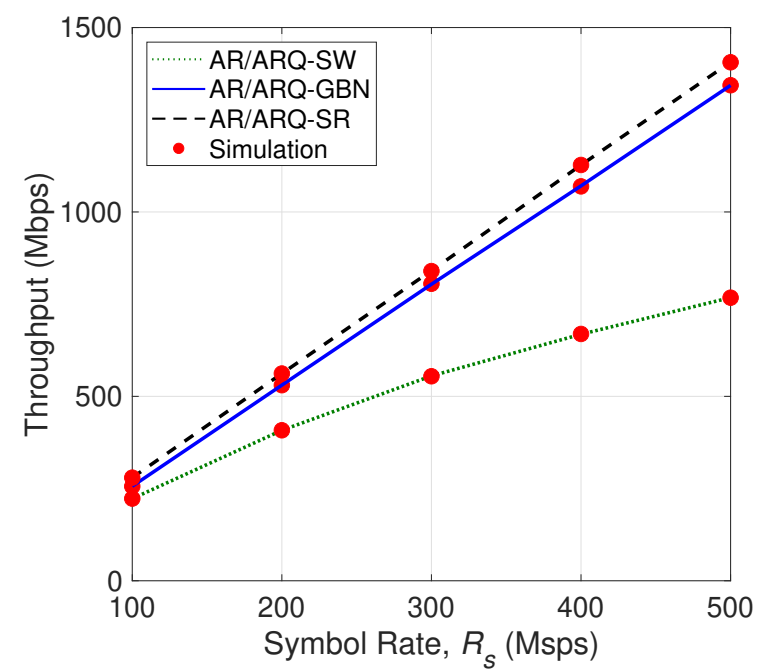

Figure 13: Throughput comparison of FSO system employing AR/ARQ-SW, AR/ARQ-GBN, and AR/ARQ-SR [34].

compared with the transmission delay, which becomes very small thanks to the high data rate of FSO systems. This leads to inefficiency in the throughput performance of AR/ARQ-SW in high-speed FSO systems.

For FSO-based satellite systems, the cross-layer design framework of AR transmission and IR-HARQ based sliding window mechanism was introduced in [133]. In this study, the IR-HARQ with AR scheme is applied to each burst, containing several frames depending on the transmission modes. From a low mother code rate of $R_{C_{N_{\mathrm{t}}}}$ (e.g., $1 / 2$ or $1 / 3$ ), a family of punctured codes, including $N_{\mathrm{t}}$ different code rates, is obtained by using the punctured technique, i.e., $(1 \geq) R_{C_{1}}>R_{C_{2}}>\cdots>$ $R_{C_{N_{\mathrm{t}}}}$. In the initial transmission round, the frames containing only the coded bits of code $R_{C_{1}}$ are selected to be transmitted in a burst. At the receiver side, after decoding for error correction with code $R_{C_{1}}$, the error detection using CRC is performed. If the presence of errors is detected, the additional redundancies for uncorrected frames are retransmitted in the next transmission round based on a NAK message indicating the sequence numbers of those frames. The newly received redundancies are then combined with the previously received frames to construct coded bits of code $R_{C_{2}}$ for decoding. The process for retransmissions of a frame is terminated when decoding the frame successfully or reaching the persistent level of IR-HARQ, i.e., $N_{\mathrm{t}}$ transmission attempts. If a frame does not get through the FSO link after $N_{\mathrm{t}}$ transmission attempts, it is clarified to be lost.

Due to using the sliding window mechanism, the design of the channel-state model is similar in [34], in which the channel is divided into equal intervals defined by a range of SNR thresholds. Nevertheless, each channel-state covers not only a burst transmission but also the feedback signals as the propagation delay is significant in long-distance satellite systems, which can not be simply ignored as in [34] for FSO terrestrial systems. Then, using the same approach in Algorithm 1, where the average channel-state duration $\bar{\tau}_{k}$ is set to $T_{\text {burst }}+2 t_{\text {prop }}$ with $t_{\text {prop }}$ the propagation delay, the SNR thresholds for channel states can be obtained. For the AR transmission (with multiple transmission modes), each channel-state is then assigned a specific transmission mode, which can bring the highest possible data rate while maintaining a targeted BER target $_{\text {at }}$ PHY. The channel-state $k$-th with the SNR interval of $\left[\gamma_{k-1}, \gamma_{k}\right), k \in\{0,1, \cdots, K\}$, is said to be in the transmission mode $n$, where $n \in\{1,2, \cdots, N\}$, if the corresponding average BER at PHY satisfies $\mathrm{BER}_{\text {target }}$. If we denote by $\phi_{k}=\{n \mid k-$ th state is assigned by mode $n\}$, the design problem can be formally formulated as

$$
\begin{array}{ll}
\underset{n=1,2, \cdots, N}{\operatorname{maximize}} & n \mid M_{\phi_{k}}=M_{n} \\
\text { subject to } & \overline{\operatorname{BER}}_{k}\left(M_{\phi_{k}}, \gamma\right) \leq \mathrm{BER}_{\text {target }},
\end{array}
$$

where $\overline{\operatorname{BER}}_{k}\left(M_{\phi_{k}}, \gamma\right)$ is the average BER at state $k$-th using mode $n$, which can be found in [133].

Example: An example of the joint design between AR scheme and IR-HARQ based sliding window mechanism is depicted in Fig. 14. Here, the window size is set to $W=1$ burst time, and the persistent level of IR-HARQ is $N_{\mathrm{t}}=3$. Also, the number of frames per burst in the transmission mode $n$ is determined by $n \times n_{\mathrm{B}}$, where $n_{\mathrm{B}}=100$ blocks/burst. In this example, frame 2 is supposed to be uncorrectable in burst 1 at the initial transmission round. The additional redundancies adopted from puncturing FEC family codes for this frame are then transmitted in the next bursts (i.e., bursts 2 and 3) together with the other new frames for the joint decoding. These redundancies are combined with the previously received frames to construct the more robust codes (i.e., lower code rates) for error correction. After reaching the HARQ's persistent level, if frame 2 is still uncorrected, it is clarified to be lost.

Figure 15 quantitatively highlights the effectiveness of the IR-HARQ protocol using a sliding window mechanism by comparing their throughputs with those of traditional IRHARQ using SW mechanism and pure sliding window ARQ over a range of received powers. A considerable throughput enhancement of link-layer solutions using sliding window mechanism compared to ones using SW mechanism is confirmed, thanks to the transmission of many frames without waiting for an acknowledgment. This phenomenon is due to the time waiting for the acknowledgment of a data frame (twice the propagation delay) in satellite communications is significant compared to the transmission delay, which becomes very small thanks to the high data rate of FSO communications. As a result, IR-HARQ based SW mechanism becomes inefficient in FSO-based satellite systems. Furthermore, as expected, the IR-HARQ protocol outperforms the pure sliding window ARQ because only incremental redundancies are required for retransmission to create a robust FEC code for frame error correction in IR-HARQ. In contrast, repetitive frames without error correction are retransmitted in the pure sliding window ARQ.

2) Link/Transport Cross-layer Design: The transmission control protocol (TCP) is, by far, the most essential transport protocol for Internet applications that require reliable delivery, e.g., HTTP, email, file transfer, and some streaming media applications [179]. TCP is the de facto standard 


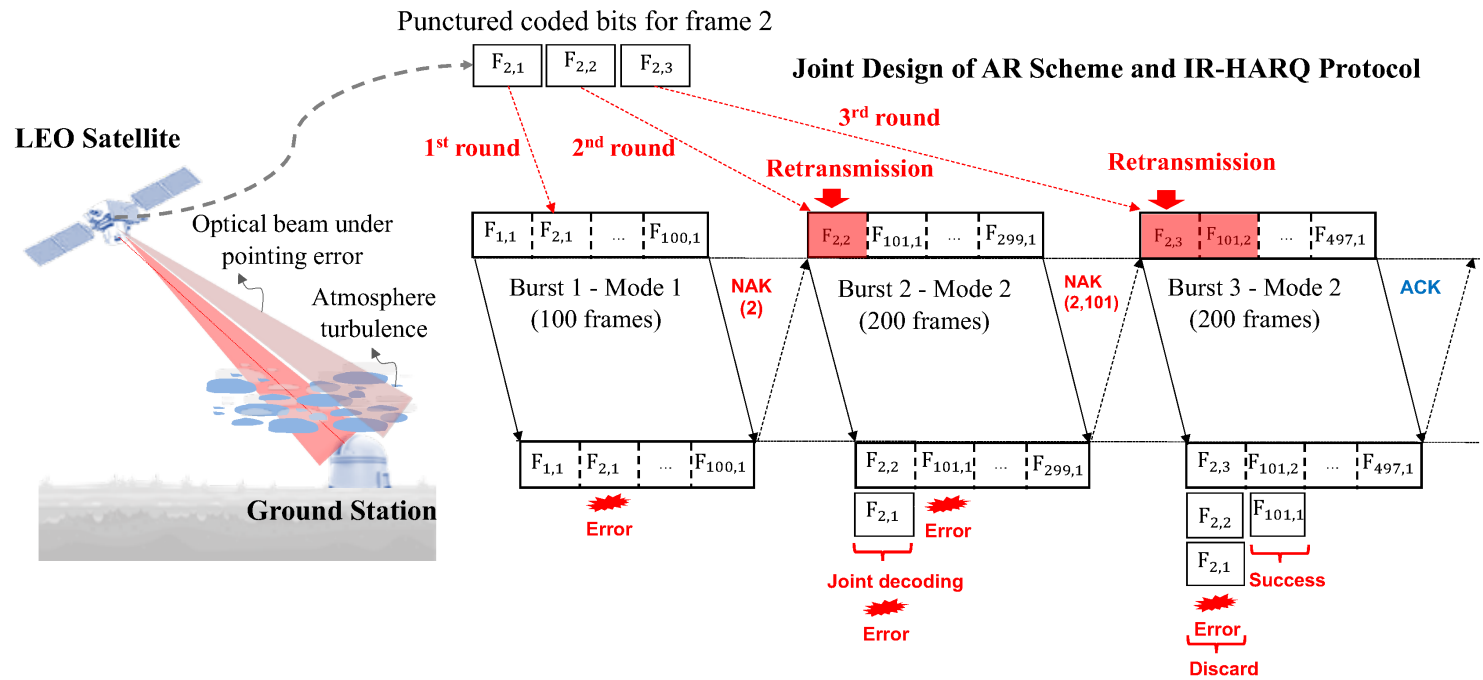

Figure 14: The proposal of satellite FSO systems using IR-HARQ based sliding window mechanism with AR scheme [133].

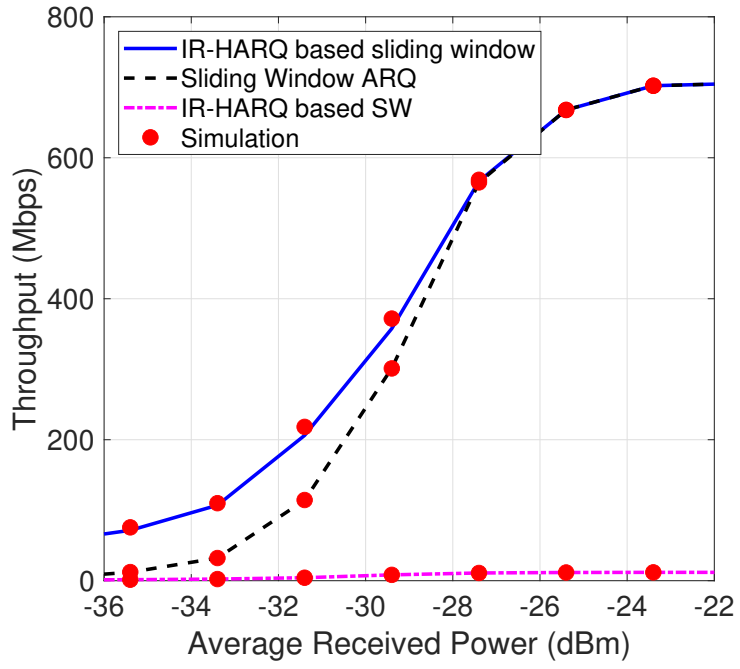

Figure 15: Throughput comparison of different link-layer solutions with AR transmission in satellite FSO systems [133].

for Internet-based commercial communication networks. It is widely known that TCP tends to perform poorly in the errorprone environment of wireless channels, primarily because it misinterprets the losses due to the poor channel conditions as an indication of network congestions [180], [181]. The issues regarding the behavior of TCP over wireless links have been extensively addressed in the domain of RF-based communication networks, e.g., [182]-[184], and recently investigated in the field of FSO networks, e.g., [185], [186].

A common approach to enhance the performance of TCPs over wireless fading channels is the use of link-layer retransmission-based error-control protocols, including ARQ [187]-[189] and HARQ [190]-[192]. The benefit of these linklayer retransmission solutions is that a transparent transmission path over wireless links is seen by TCP variants, provided most wireless channel errors can be mitigated by link-layer error control schemes. Driven by that fact, in the domain of

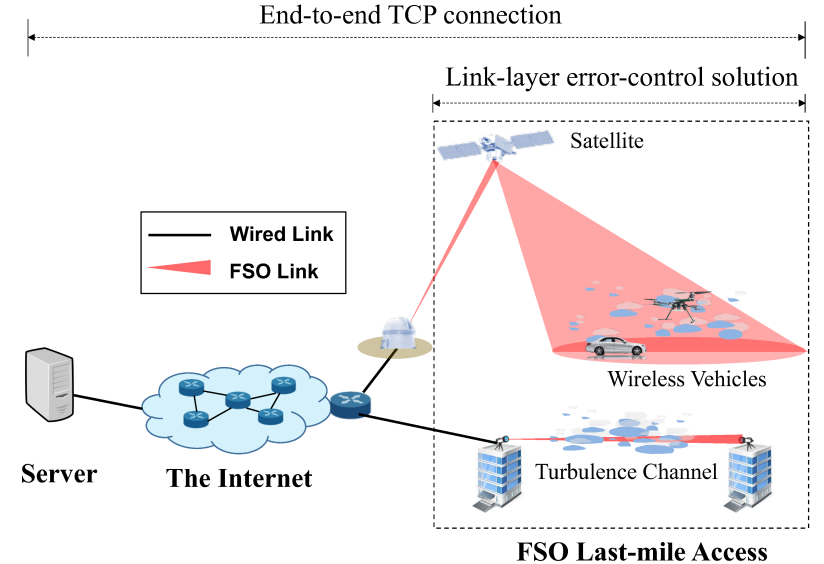

Figure 16: End-to-end network scenario with TCP connections and FSO last-mile access.

FSO-based communication networks, many research efforts have addressed the cross-layer design between link-layer retransmission protocols and TCP variants to improve the TCP throughput performance over the FSO last-mile access [162], [167]-[171]. As shown in Fig. 16, the last-mile access could be an FSO link from satellite to provide the Internet connectivity for wireless vehicles, e.g., autonomous cars and unmanned aerial vehicles (UAVs) or between buildings.

The joint design of link-layer SR-ARQ protocol with standard TCP variants, including TCP Tahoe, TCP Reno, and TCP-Selective Acknowledgement (SACK), over FSO-based terrestrial networks, was studied in [167]. Each TCP segment is divided into $L_{\mathrm{f}}$ smaller link-layer data frames before being transmitted over the FSO turbulence channels. The objective of the SR-ARQ protocol is to mitigate the transmission errors caused by the atmospheric turbulence channels by retransmitting the corrupted frames, thus improving the TCP throughput performance. Here, if a link-layer frame does not get through the FSO link after the SR-ARQ's persistent level, i.e., $N_{\mathrm{t}}$, SR-ARQ gives up, and the corresponding TCP segment is 
clarified to be lost. While a higher value of ARQ persistent level can reduce the effects of frame losses or segment losses and increase the TCP throughput, it also increases the overall end-to-end latency or round-trip time (RTT) of TCP. The TCP segment loss probability (SLP) and average RTT can be written as [167]

$$
\begin{gathered}
\mathrm{SLP}=1-\underbrace{\left(1-P_{\text {others }}\right)}_{\text {Other parts }} \times \underbrace{\left(1-\mathrm{FER}^{N_{\mathrm{t}}}\right)^{L_{\mathrm{f}}}}_{\text {FSO last-mile }}, \\
\mathbb{E}[\mathrm{RTT}]=\underbrace{2 t_{\text {others }}}_{\text {RTT of other parts }}+\underbrace{\left(2 t_{\text {prop }}+N_{\mathrm{f}} / R_{\mathrm{b}}\right) \bar{N}_{\mathrm{t}} L_{\mathrm{f}}}_{\text {RTT of FSO last-mile }},
\end{gathered}
$$

where $\mathbb{E}(\cdot)$ denote the expected value, $P_{\text {others }}$ is the SLP over other parts of network, $N_{\mathrm{f}}$ is the frame size, $R_{\mathrm{b}}$ is the transmission bit rate, and $\bar{N}_{\mathrm{t}}$ is the average number of retransmissions of a frame. From the tradeoff between TCP throughput and end-to-end latency, the ARQ's persistent level was suggested to be $N_{\mathrm{t}}=6$ for the considered FSO networks in [167].

The critical weakness of the cross-layer framework in [167] is, however, the use of a uniform error model, which may not accurately estimate the TCP throughput performance. For such models, the error structure of different frame transmissions is assumed to be independent. In fact, in FSO systems, during the transmission within a coherence time of turbulence fading channel (i.e., order of milliseconds [90]), the frame errors tend to occur in a burst pattern, and the error structure is correlated. It is, therefore, essential to investigate the correlated error transmission structure to provide a more accurate performance analysis of TCP. The cross-layer design framework incorporating the AMC scheme at the physical layer, SR-ARQ protocol at the link-layer, and standard TCP variant (i.e., TCP Reno) at the transport layer, was investigated in [162]. Also, instead of using the uniform error model, a more accurate performance analysis of TCP was provided, taking into account the nature of burst error by using a Markov error model. A considerable TCP throughput improvement was observed in [162] by jointly design of lower layers protocols, i.e., both AMC scheme and SR-ARQ protocol.

As a matter of fact, the critical advantage of the analytical approach in [162] is the provision of a good approximation of network performance with various channel conditions in a relatively quick fashion, which supports the optimization purposes. However, simplifying assumptions are often used at the cost of accuracy for mathematical modeling, e.g., the slowstart phase is usually ignored in the TCP performance analysis [193]. Another approach for the TCP performance evaluation is the use of actual measurements, which offers better accuracy [186]. The main drawback of this approach is that it is not straightforward to obtain generalized results requiring specific measurements in various environmental conditions, which is, in fact, time-consuming, costly, and not always possible in many cases. As a result, the popular network simulator, i.e., NS-2 [194], which allows an accurate evaluation of a wide range of network protocols, was used to evaluate the performance of TCP over FSO turbulence channels in the presence of PHY error models and SR-ARQ protocols [168]. Using ARQ protocols, the maximum TCP's window size could be maintained over time since retransmissions at the link-layer considerably reduce the TCP's SLP.

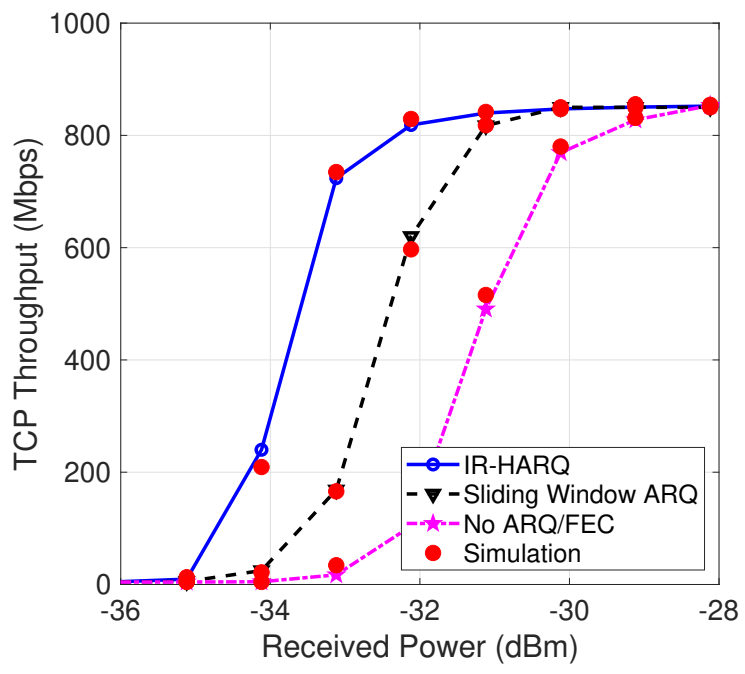

Figure 17: TCP throughput performance in FSO-based satellite networks for different link-layer solutions [169].

In the context of FSO-based satellite-assisted IoVs networks, as reported in [133] for link-layer error-control solutions, the HARQ protocol is preferable compared to the pure ARQ protocol due to high achievable throughput performance. A cross-layer design framework between IR-HARQ based sliding window protocol and TCP variants, including Cubic and Hybla, was mentioned for the scenario of satellite-assisted Internet of vehicles in [169], [170]. Similar to [162], a burst loss model was developed for the cross-layer performance analysis, which can accurately estimate the TCP throughput performance. Figure 17 quantitatively compares the use of different link-layer solutions, including IR-HARQ, pure sliding window ARQ, and no ARQ/FEC, for the throughput performance of TCP Cubic [169]. As expected, the TCP throughput performance could be significantly enhanced when using the link-layer IR-HARQ based sliding window protocol, which requires retransmit only the incremental redundancies for erroneous frames. It can mitigate the effect of transmission losses compared to the case of no ARQ/FEC and reduce the number of retransmissions compared to using pure sliding window ARQ, thus increasing the achievable TCP throughput performance.

Another approach to improve the TCP throughput performance for such satellite-assisted high-speed vehicular networks is using a combination of PHY FEC code and link-layer ARQ protocol [171]. In this study, a novel cross-layer design framework incorporating the PHY FEC code, link-layer SRARQ protocol, and TCP variants at the transport layer, was developed for the scenario of satellite-based hybrid $\mathrm{FSO} / \mathrm{RF}$ vehicular networks. Furthermore, unlike analytical models in [162], [167], [169], [170], where only the congestion avoidance phase was considered, a complete model taking into account other phases of TCP operation was mentioned in [171]. Using the SR-ARQ protocol, which processes multiple frames simultaneously, a channel-state model was developed 


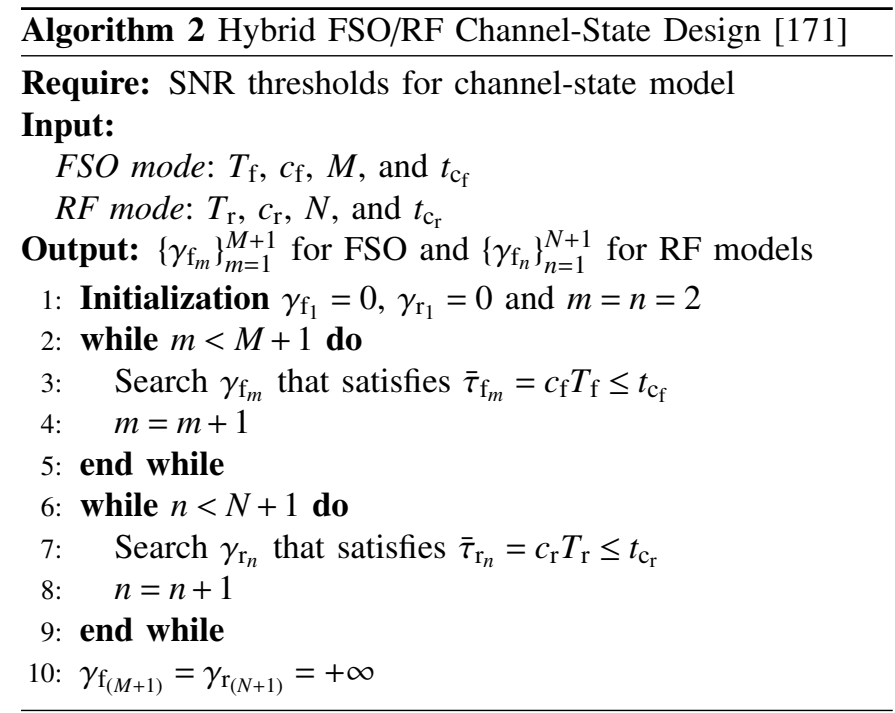

to facilitate the frame transmissions over hybrid FSO/RF channels. The duration of the channel-state is selected long enough for the transmission of multiple frames, and it should be equal and shorter than the channel fading coherence time, i.e., $t_{\mathrm{c}_{\mathrm{f}}}$ and $t_{\mathrm{c}_{\mathrm{r}}}$ for FSO and RF channels, respectively. Both FSO and RF channels are organized into non-overlapping consecutive states, defined by a range of SNR thresholds. The design of a channel-state model for hybrid FSO/RF systems to facilitate the operation of SR-ARQ protocols is summarized in Algorithm 2, where $T_{\mathrm{f}}\left(T_{\mathrm{r}}\right)$ is the frame duration in the FSO (RF) mode, $c_{\mathrm{f}}\left(c_{\mathrm{r}}\right)$ (frames/state) is the number of frames in each FSO (RF) channel-state decided by designers, and $M(N)$ is the total number of channel-state in the FSO (RF) mode. In addition, $\bar{\tau}_{\mathrm{f}_{m}}$ and $\bar{\tau}_{\mathrm{r}_{n}}$ are respectively the average channel-state duration of FSO and RF modes, which can be found in [195].

\section{Findings, Challenges, and Open Issues}

This section presents our findings as a result of the comprehensive survey of link-layer retransmission-based error-control protocols, both ARQ and HARQ, and their cross-layer design frameworks in the context of high-speed FSO communications. First, we elaborate on the lessons learned from the aforementioned survey of the literature. Then, we suggest the design guidelines for developing link-layer retransmission protocols in various FSO network scenarios. Finally, we identify and discuss the non-exhaustive list of research challenges, then shed light on future research directions and applications.

\section{A. Lessons Learned}

From the comprehensive literature for design and performance evaluation of ARQ protocols in the context of FSO communications in Section II, we now identify several valuable lessons learned regarding such protocols.

- The ARQ protocol, which is sufficiently reliable yet simple, and has less overhead, is an efficient error-control solution for short-range FSO systems. As reported in [33], there are two important reasons. Firstly, the impact of channel impairments in short-range FSO systems is less severe than that of the RF; hence using another link-layer robust method, e.g., HARQ protocols, results in more system complexity, additional signaling, and overhead. Secondly, with the aid of PHY error-control methods, e.g., FEC and/or AR schemes, the latency caused by the ARQ's retransmissions for residual errors in short-range FSO communications does not significantly degrade the system performance. In long-range FSO systems, the ARQ protocol is an inefficient solution. In fact, in the case of high latency as in satellite/aerial or deep-space FSO systems, the performance of such protocols is severely deteriorated due to the increased latency.

- The SW-ARQ protocol is not practically valuable for high-speed FSO systems, and sliding window protocols are preferable for such systems. As a matter of fact, the waiting time for the acknowledgment of a data frame, i.e., twice the propagation delay, in SW-ARQ protocol becomes significant compared with the transmission delay, which is minimal thanks to the high data rate of FSO communications, as illustrated in [34]. This results in inefficiency in terms of the throughput performance of SW-ARQ in FSO systems. As a result, sliding window protocols, which allow higher efficiency by letting data frames be continuously transmitted without waiting for acknowledgment, are considered an effective solution for such FSO systems.

- The joint design of $A R Q$ protocols and adaptive transmissions, i.e., data-rate or coding-rate, could significantly enhance the FSO system performance. The existing studies on the integration between ARQ protocols and adaptive transmissions confirmed the effectiveness in improving the overall system performance over atmospheric turbulence channels, e.g., [33], [64]. In fact, the main objective of the adaptive transmission is to maintain a required QoS, e.g., targeted FER, by adaptively adjusting parameters (e.g., modulation size and coding rate) according to the channel conditions. By retaining a low level of transmission error rate over the turbulence-induced channels, ARQ protocols, therefore, become efficient and could effectively improve the FSO system performance.

- ARQ protocols using the concept frame combining can offer considerable performance improvement without introducing a significant system cost increase. As introduced in [33], the ARQ-based FC realized by implementing frame combining at the receiver could be used to improve the reliability of standard-ARQ protocols. The main idea behind this scheme is to buffer the copies of previously received data frames for joint decoding, which enhances the likelihood of successful frame decoding.

- The adaptive power allocation between the $A R Q$ retransmissions significantly improves the performance of hybrid FSO/RF systems. This fact was confirmed in [71] for the design of ARQ protocols in hybrid FSO/RF communications based on parallel/simultaneous FSO and RF transmissions. The intuition behind the considered power allocation is to weigh the energy in each ARQ's round by its consumption probability. Additional energy is assigned to the last retransmissions, which are rarely 
used.

- The current design of $A R Q$ protocols in the context of FSO communications is mainly based on the target throughput performance. In other words, most of the studies in the literature focus on the throughput performance without considering other performance metrics, e.g., latency and energy efficiency, which are especially essential for FSO-based satellite/aerial systems.

Next, we highlight the following lessons learned from the design and performance perspective of HARQ protocols over FSO turbulence-induced fading channels reviewed in Section III.

- $H A R Q$ protocols are preferable to the pure $A R Q$ in longrange FSO systems. As a matter of fact, pure ARQ protocols, which facilitate the retransmissions of repetitive data frames, often fail to provide satisfactory delay and throughput performance over time-varying channel conditions, as many retransmissions are required. Benefited from their inherent advantages, HARQ schemes are an indispensable and effective solution to maintain reliable transmissions for such long-range FSO systems.

- The IR-HARQ protocol is the most efficient solution for various FSO communication scenarios. Although the CC scheme could enhance the link performance by coherently combining multiple copies of the received frames, the IR strategy can benefit from these as well as certain coding gains. The reason is that the different coded frames can jointly form a lower code rate with more powerful error protection capabilities.

- It is not possible to jointly maximize the energy efficiency and throughput performance in FSO systems using HARQ protocols. In fact, when the transmitted powers are high enough, the FER saturates, and any further increase of transmitted power only leads to additional energy consumptions. As a result, an optimal value of transmitted power exists, at which the energy efficiency is maximized. However, these values are not always the optimal ones for throughput performance, in which the tradeoff between them was investigated in [133] under various channel conditions.

- The performance of HARQ protocols in FSO-based terrestrial systems can be analyzed from an informationtheoretic perspective. In general, the performance analysis of HARQ protocols, which depends on the types of codes and decoding/error detection technique, is based on the Markov chain. Modeling the system by a Markov model might be complicated since each state must convey all the information about the memory of systems [196]. As a result, the information-theoretic approach [197] could be used to gain insight into the implication on the performance of HARQ protocols, resorting to the relatively simple models which lend themselves to the analytical investigation.

- The impact of imperfect estimated CSIs significantly degrades the performance of FSO systems using adaptive $H A R Q$ protocols. Indeed, the availability of up-todate CSIs at the transmitter plays an essential role in adapting the code rate of HARQ's transmission rounds, thereby achieving maximum throughput and reducing the latency due to retransmissions [126], [127]. As a result, the investigation of practically imperfect CSIs on the performance of HARQ systems is critical, especially for long-distance FSO communications.

- The HARQ-based protocol using rateless codes is a promising solution for hybrid FSO/RF systems. As presented in [120], HARQ protocols using the rateless codes adapt seamlessly to the changes in rate supported by the channels without the rate mismatch due to the imprecise/outdated channel estimation. This is a distinct performance benefit over the conventional HARQ-based code-rate selection, which may fail if the channel conditions vary notably from one codeword to another.

- The inter-user cooperative HARQ protocol is an effective solution for broadcasting FSO systems. For such broadcasting systems, instead of relying on the center node, a user, who successfully decoded the original data frame and whose distance from the NAK issuing user is shorter than that of the center node, is invited to retransmit this frame to the latter user. This inter-user cooperative HARQ scheme could offer a considerable performance improvement compared to the conventional cooperative HARQ protocol confirmed in [131].

Finally, the lessons learned from the cross-layer design frameworks of link-layer retransmission protocols with PHY methods and/or transport layer protocols presented in Section IV can be summarized as follows.

- The cross-layer design between link-layer retransmission protocols and AR transmission is an effective solution for FSO communications. Such cross-layer framework has recently received significant attention due to two remarkable reasons. Firstly, the channel fading in FSO systems is slowly varying. Due to this quasi-static channel property, the CSI for AR transmission can be estimated accurately and fed back to the transmitter, making AR schemes more popular among PHY solutions. Secondly, the integration between such methods could offer a considerable performance enhancement with no additional physical infrastructure required.

- The design of the channel-state model considering the FSO channel coherence time could effectively facilitate the operation of ARQ/HARQ-based sliding window mechanism with AR transmission. For such a crosslayer design, the data is transmitted in fixed-time bursts containing multiple frames. The channel-state model, defined by a range of SNR thresholds, is then designed to cover the burst transmissions, in which the interval of all channel states are equal to the burst duration and shorter than the FSO fading channel coherence time.

- Link-layer retransmission protocols could effectively improve the performance of TCP variants, in which the selection of their persistent level should be considered carefully. As a matter of fact, higher values of the persistent level of ARQ/HARQ protocols can mitigate the impact of frame losses caused by FSO turbulence-induced 
fading channels, thus improving the TCP throughput performance. However, such a high value of persistent level also increases the overall end-to-end latency of TCP in networks. As a result, proper selection of persistent levels for $\mathrm{ARQ} / \mathrm{HARQ}$ protocols plays an essential role in effectively optimizing the overall performance of TCPs.

- Developing a link-layer error model, which illustrates the correlated frame error transmission structure, is essential to provide a more accurate performance analysis of TCP. In FSO communications, frame transmission errors tend to occur in a burst pattern during a coherence time of turbulence fading channels, and the error structure is correlated. Therefore, the traditional uniform error model does not reflect this critical issue, leading to inaccurate TCP throughput performance evaluation over turbulenceinduced fading channels.

- The IR-HARQ based sliding window mechanism is the most efficient link-layer solution in improving the throughput performance of TCPs in the context of satellite-based FSO networks. Thanks to retransmission of only incremental redundancies to create a more robust code to correct erroneous frames, IR-HARQ protocols can mitigate the effect of transmission losses compared to the case of no ARQ/ECC. Furthermore, compared with pure sliding window ARQ protocols, it also reduces the number of retransmissions required, thus increasing the achievable throughput performance of TCPs.

- The joint design of RS code at PHY and link-layer $S R-A R Q$ protocol could offer a significant throughput improvement of TCP variants in the context of satellitebased hybrid FSO/RF vehicular networks. This fact has been recently confirmed in [171]. By using RS codes at PHY, the transmissions can be maintained at low errorrate levels, and retransmission protocols operating at linklayer could effectively mitigate the residual errors from the PHY layer.

\section{B. Design Guidelines}

We now attempt to provide a design guideline of linklayer retransmission protocols recommended for several FSO scenarios, as explicitly shown in Fig. 18. It is noteworthy that this guideline comprises plausible and reasonable observations gleaned from the intensive survey of ARQ, HARQ, and the cross-layer designs, in the context of FSO communications. Based on the trade-off between complexity and performance, our recommendations for such protocol designs in different FSO network scenarios are listed below.

- Short-range FSO systems: According to the investigations in [34], [72], [73], the joint design of AR transmissions and sliding window protocols, i.e., either GBN-ARQ or SR-ARQ, could offer a significant throughput improvement over turbulence-induced fading channels. While SR-ARQ can provide a higher throughput performance than GBN-ARQ, it is more complex in terms of implementation. In addition, as shown in [73, Fig. 6], their throughputs were close in weak turbulence conditions. Hence, we recommend the use of $A R / G B N-A R Q$ and
$\mathrm{AR} / \mathrm{SR}-\mathrm{ARQ}$ in respectively weak and strong turbulence conditions for short-range FSO communications, e.g., building-to-building.

- Long-range FSO systems: For HAP-to-HAP and satelliteto-satellite FSO systems, which are less susceptible to weather, such as atmospheric turbulence and clouds, the adaptive HARQ protocols are suitable, as reported by DLR [126], [127]. In addition, it is suggested to employ a joint design of AR schemes and HARQ-based sliding window mechanism for long-range FSO systems under the impact of atmospheric turbulence, e.g., those with satellite-to-vehicles and ground-to-satellite links. As highlighted in [133], the joint design of the AR/CCHARQ and AR/IR-HARQ could offer a considerable performance enhancement, including throughput, energy efficiency, and delay, for satellite-based FSO systems.

- Hybrid FSO/RF systems: Both ARQ-based adaptive power allocation [71] and HARQ-based rateless codes [120] are promising candidates for hybrid FSO/RF systems. Notably, the former is based on the power allocation for retransmission rounds of ARQ, while the latter uses the concept of rateless code for HARQ, which does not require rate adjustment before transmissions.

- Cooperative FSO systems: As introduced in [131], the inter-user cooperative HARQ protocol, using the idea of frame retransmissions by neighboring nodes instead of the center one, is an efficient solution for FSO broadcasting systems. In addition, the M-C-ARQ protocols investigated in [63] can achieve a better performance than the conventional C-ARQ ones in the context of cooperative FSO-based terrestrial systems. Regarding the high-latency satellite-based FSO communications, the use of cooperative IR-HARQ is preferable to maintain high performance, as confirmed in [134] for HAP-based relaying satelliteto-vehicles FSO systems.

\section{Challenges and Open Issues}

We now identify the research challenges and discuss the open issues for link-layer retransmission protocols in the vision of future sixth-generation $(6 \mathrm{G})$ wireless networks. Interested readers can refer to recent papers discussing the vision, applications, requirements, and technologies for future $6 \mathrm{G}$ wireless networks [198]-[201]

1) Research Challenges: To enhance the reliability of FSO systems, link-layer retransmission-based error-control protocols, both ARQ and HARQ, have attracted much attention in recent years. Novel designs of such protocols for high-speed FSO communications have been investigated to offer better system performance. Nevertheless, there are some challenges that researchers need to overcome to meet the demand of future wireless networks.

- Latency: Massive ultra-reliable and low latency communication (mURLLC) is one of the demanding requirements for future $6 \mathrm{G}$ wireless networks [202]. Although link-layer retransmission protocols can fully exploit time diversity to increase reliability, they usually suffer from high latency caused by multiple retransmissions, multiple 


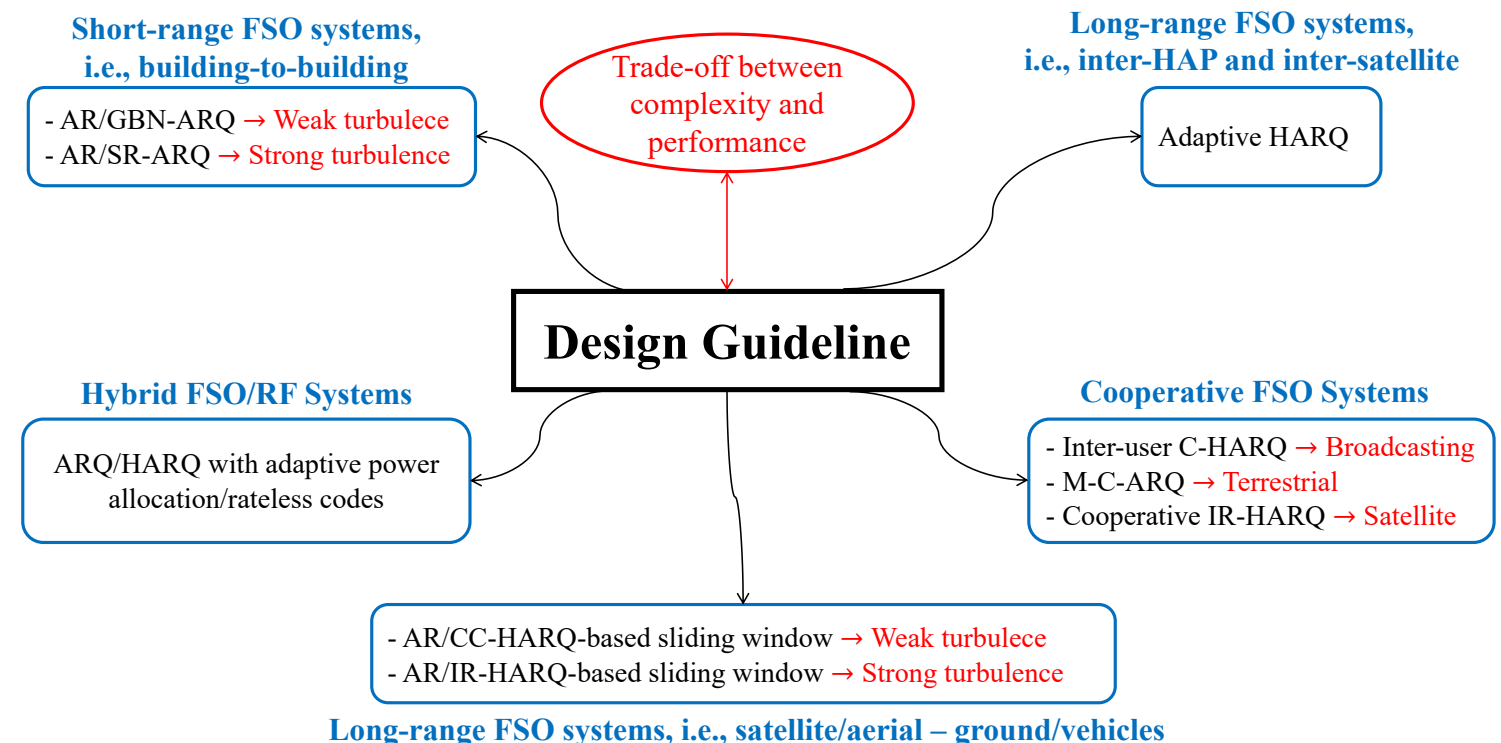

Figure 18: Design guidelines of link-layer retransmission protocols recommended for several FSO communication scenarios.

frames decoding at the receiver, and the ACK/NAK signals transmission/processing delay [203]. In fact, it is difficult to support, for example, more than one retransmission within 1 ms end-to-end latency constraint of mURLLC at least for the initial mURLLC specification in Rel. 15 [154]. Reducing the end-to-end latency to a minimum for enabling ARQ/HARQ protocols in mURLLC becomes a critical issue. Hence, it is challenging to design such protocols to meet the requirement of mURLLC.

- Energy Efficiency: Regarding the demand of global coverage (near $100 \%$ geographical coverage) for $6 \mathrm{G}$ wireless networks, non-terrestrial networks, including satellites, HAPs, and UAVs, are needed to complement the terrestrial networks for the cost-effective, seamless, and ubiquitous service availability [204]. Indeed, energy-efficient communications reduce energy consumptions and extend the battery life of wireless terminals, especially crucial in FSO-based satellite/aerial systems (with limited powers) [205]. Achieving a low transmission error rate by using retransmission protocols may require high energy consumption due to frequent retransmissions. Therefore, green communications for 6G-based non-terrestrial networks become a challenging issue for the design of linklayer retransmission protocols.

- Security: Security and privacy are vital to the success of envisioned 6G wireless networks [206]. As reported in [207], retransmission is an effective way to improve reliability, but it may also compromise security due to the provision of additional diversity for eavesdroppers. For instance, using HARQ protocols, the transmitter needs to provide sufficient redundancies for the legitimate receiver to decode its message successfully. Too many redundancies, nevertheless, may help the adversarial eavesdropping [208]. On the other hand, thanks to the wide coverage beam footprint, FSO-based satellite communications can support multiple users within a single laser beam [25].
For such network scenarios, security is one of the critical concerns in the design of link-layer retransmission protocols.

2) Open Issues: From the aforementioned research challenges, we discuss some potential open research issues that deserve attention for link-layer retransmission protocols in the context of FSO communication networks.

- Machine Learning for Early ARQ/HARQ: Strategies for reducing the feedback delay using prediction mechanisms powered by machine learning techniques have been recently studied in the context of RF-based wireless communications [154], [209], [210]. From the machine learning perspective, the main task is to predict the decoding result of a given transmission using data, which is available after the first few decoder iterations. In the context of FSO communications, it is worth noting that machine learning in ARQ/HARQ protocols has not been well investigated yet. Indeed, extensive research is needed to exploit the potential of machine learning to enhance the performance of link-layer retransmission protocols. Machine learning for retransmission protocols in highspeed FSO systems would certainly be a hot topic and raise interest for future studies.

- Quantum $A R Q / H A R Q$ : In the fast technological advances over the last couple of decades, quantum technology has emerged as a promising candidate that has the potential of radically revolutionizing the way we compute and communicate [211]-[213]. Quantum communications support secure data dissemination since any measurement or observation by eavesdroppers perturbs the quantum superposition. Real-word quantum channels, as well as quantum systems, are, nevertheless, not perfect, in which quantum bits (qubits) may experience both channelinduced and quantum processing impairments [212]. For instance, the secret key transmission rate of a quantum key distribution $(\mathrm{QKD})$ system deteriorates significantly 
over the turbulence-induced fading channels [214]. One of the essential prerequisites to build reliable quantum communications is the employments of error-control solutions. However, the law of quantum mechanics prevents us from transplanting classical error-control protocols directly into the quantum domain. The initial studies on quantum ARQ/HARQ protocols for quantum communication systems have been reported in [215], [216]. Extensive investigation is needed to exploit the potential quantum ARQ/HARQ protocols in quantum communication systems.

\section{Conclusions}

This paper presents a state-of-the-art survey on the design and performance evaluation of link-layer retransmission-based error-control protocols, both ARQ and HARQ, in the emerging high-speed FSO communication networks. The survey was conducted extensively in various FSO communication scenarios, including point-to-point terrestrial, cooperative, multihop relaying, hybrid FSO/RF, satellite/aerial, and deep-space systems. Also, we provided a survey of recent works and insightful discussion on the cross-layer design frameworks related to link-layer retransmission protocols in FSO communication networks. The critical lessons from the survey, followed by potential research directions for each domain, have been derived. Moreover, we derived a design guideline comprising plausible and reasonable observations gleaned from the intensive survey of ARQ, HARQ, and cross-layer design frameworks. In addition, based on the holistic survey, we have pointed out the fundamental research challenges to be considered carefully for further investigation of such protocols in the context of future $6 \mathrm{G}$ wireless communication networks. Finally, we have discussed and outlined potential open issues toward future $6 \mathrm{G}$ wireless networks.

\section{REFERENCES}

[1] F. Tang, Y. Kawamoto, N. Kato, and J. Liu, "Future intelligent and secure vehicular network toward 6g: Machine-learning approaches," Proc. IEEE, vol. 108, no. 2, pp. 292-307, Dec. 2020.

[2] ITU, "The data traffic estimates for the years 2020 to 2030." [Online] Available: https://www.itu.int/pub/R-REP-M.2370-2015s.

[3] Z. Ghassemlooy, S. Arnon, M. Uysal, Z. Xu, and J. Cheng, "Emerging optical wireless communications-advances and challenges," IEEE $J$. Sel. Areas Commun., vol. 33, no. 9, pp. 1738-1749, Jul. 2015.

[4] M. Li, "Orbital-angular-momentum multiplexing optical wireless communications with adaptive modes adjustment in internet-of-things networks," IEEE Internet Things J., vol. 6, no. 4, pp. 6134-6139, Aug. 2019.

[5] L. E. M. Matheus, A. B. Vieira, L. F. M. Vieira, M. A. M. Vieira, and O. Gnawali, "Visible light communication: Concepts, applications and challenges," IEEE Commun. Surveys Tuts., vol. 21, no. 4, pp. 3204 3237, Apr. 2019.

[6] M. Z. Chowdhury, M. K. Hasan, M. Shahjalal, M. T. Hossan, and Y. M. Jang, "Optical wireless hybrid networks: Trends, opportunities, challenges, and research directions," IEEE Commun. Surveys Tuts., vol. 22, no. 2, pp. 930-966, Jan. 2020.

[7] A. S. Hamza, J. S. Deogun, and D. R. Alexander, "Classification framework for free space optical communication links and systems," IEEE Commun. Surveys Tuts., vol. 21, no. 2, pp. 1346-1382, Oct. 2019.

[8] E. Yaacoub and M. Alouini, "A key $6 \mathrm{~g}$ challenge and opportunityconnecting the base of the pyramid: A survey on rural connectivity," Proc. IEEE, vol. 108, no. 4, pp. 533-582, Mar. 2020.

[9] D. R. Kolev and M. Toyoshima, "Received-power fluctuation analysis for LEO satellite-to-ground laser links," J. Lightwave Technol., vol. 35, no. 1, pp. 103-112, Nov. 2017.
[10] S. A. Al-Gailani, M. F. Mohd Salleh, A. A. Salem, R. Q. Shaddad, U. U. Sheikh, N. A. Algeelani, and T. A. Almohamad, "A survey of free space optics (FSO) communication systems, links, and networks," IEEE Access, vol. 9, pp. 7353-7373, Jan. 2021.

[11] I. B. Djordjevic, "Adaptive modulation and coding for free-space optical channels," IEEE/OSA J. Opt. Commun. Netw., vol. 2, no. 5, pp. 221-229, Apr. 2010.

[12] N. D. Chatzidiamantis, A. S. Lioumpas, G. K. Karagiannidis, and S. Arnon, "Adaptive subcarrier psk intensity modulation in free space optical systems," IEEE Trans. Commun., vol. 59, no. 5, pp. 1368-1377, Mar. 2011

[13] M. Z. Hassan, M. J. Hossain, and J. Cheng, "Performance of nonadaptive and adaptive subcarrier intensity modulations in gamma gamma turbulence," IEEE Trans. Commun., vol. 61, no. 7, pp. 29462957, 2013.

[14] M. Karimi and M. Uysal, "Novel adaptive transmission algorithms for free-space optical links," IEEE Trans. Commun., vol. 60, no. 12, pp. 3808-3815, Sep. 2012.

[15] H. Safi, A. A. Sharifi, M. T. Dabiri, I. S. Ansari, and J. Cheng, "Adaptive channel coding and power control for practical FSO communication systems under channel estimation error," IEEE Trans. Veh. Technol., vol. 68, no. 8, pp. 7566-7577, May 2019.

[16] W. Gappmair and M. Flohberger, "Error performance of coded FSO links in turbulent atmosphere modeled by gamma gamma distributions," IEEE Trans. Wireless Commun., vol. 8, no. 5, pp. 2209-2213, May 2009.

[17] A. T. Pham, T. A. Luu, and N. T. Dang, "Performance bound for turbo-coded 2-D FSO/CDMA systems over atmospheric turbulence channels," IEICE Trans. Fundamentals, vol. E93.A, no. 12, pp. $2696-$ 2699, Dec. 2010.

[18] T. A. Luu, N. T. Dang, and A. T. Pham, "Channel coding for 2-D FSO/CDMA systems over strong atmospheric turbulence channels," in Proc. IEEE Int. Conf. Comm. Electron., pp. 278-283, 2010.

[19] J. Fang, M. Bi, S. Xiao, G. Yang, L. Liu, Y. Zhang, and W. Hu, "Polarcoded mimo fso communication system over gamma gamma turbulence channel with spatially correlated fading," IEEE/OSA J. Opt. Commun. Netw., vol. 10, no. 11, pp. 915-923, Nov. 2018.

[20] M. Safari, M. M. Rad, and M. Uysal, "Multi-hop relaying over the atmospheric poisson channel: Outage analysis and optimization," IEEE Trans. Commun., vol. 60, no. 3, pp. 817-829, Jan. 2012.

[21] E. Zedini, H. Soury, and M. Alouini, "Dual-hop FSO transmission systems over gamma gamma turbulence with pointing errors," IEEE Trans. Wireless Commun., vol. 16, no. 2, pp. 784-796, Nov. 2017.

[22] T. V. Pham and A. T. Pham, "Performance analysis of amplify-decodeand-forward multihop binary phase-shift keying/free-space optical systems using avalanche photodiode receivers over atmospheric turbulence channels," IET Commun., vol. 8, no. 9, pp. 1518-1526, Jun. 2014.

[23] C. Abou-Rjeily and S. Haddad, "Cooperative FSO systems: Performance analysis and optimal power allocation," J. Lightw. Technol., vol. 29 , no. 7 , pp. 1058-1065, Feb. 2011.

[24] T. V. Pham, T. C. Thang, and A. T. Pham, "Average achievable rate of spatial diversity MIMO-FSO over correlated gamma gamma fading channels," IEEE/OSA J. Opt. Commun. Netw., vol. 10, no. 8, pp. 662674, Aug. 2018.

[25] H. D. Le and A. T. Pham, "Level crossing rate and average fade duration of satellite-to-UAV FSO channels," IEEE Photon. J., vol. 13, no. 1, pp. 1-14, Feb. 2021.

[26] V. Jamali, D. S. Michalopoulos, M. Uysal, and R. Schober, "Link allocation for multiuser systems with hybrid RF/FSO backhaul: Delaylimited and delay-tolerant designs," IEEE Trans. Wireless Commun., vol. 15 , no. 5, pp. 3281-3295, Jan. 2016.

[27] M. Najafi, V. Jamali, and R. Schober, "Optimal relay selection for the parallel hybrid RF/FSO relay channel: Non-buffer-aided and bufferaided designs," IEEE Trans. Commun., vol. 65, no. 7, pp. 2794-2810, Apr. 2017.

[28] M. Z. Hassan, M. J. Hossain, J. Cheng, and V. C. M. Leung, "Hybrid RF/FSO backhaul networks with statistical-QoS-aware buffer-aided relaying," IEEE Trans. Wireless Commun., vol. 19, no. 3, pp. 14641483 , Oct. 2020

[29] M. L. B. Riediger, R. Schober, and L. Lampe, "Fast multiple-symbol detection for free-space optical communications," IEEE Trans. Commun., vol. 57, no. 4, pp. 1119-1128, Apr. 2009.

[30] N. D. Chatzidiamantis, G. K. Karagiannidis, and M. Uysal, "Generalized maximum-likelihood sequence detection for photon-counting free space optical systems," IEEE Trans. Commun., vol. 58, no. 12, pp. 3381-3385, Oct. 2010. 
[31] Y. Hong, W. Shin, and S. Han, "Performance of scintillation mitigation for linear polarization shift on-off keying transmission in free-space optical communications," IEEE Access, vol. 8, pp. 128954-128960, Jul. 2020.

[32] J. Liu, X. Zhang, K. Blow, and S. Fowler, "Performance analysis of packet layer FEC codes and interleaving in FSO channels," IET Comm., vol. 11, no. 13, pp. 2042-2048, Oct. 2017.

[33] V. V. Mai and A. T. Pham, "Cross-layer designs and analysis of adaptive-rate transmission and ARQ for free-space optical communications," IEEE Photon. J., vol. 8, no. 1, pp. 1-15, Feb. 2016.

[34] H. D. Le, V. V. Mai, C. T. Nguyen, and A. T. Pham, "Design and analysis of sliding window ARQ protocols with rate adaptation for burst transmission over FSO turbulence channels," IEEE/OSA J. Opt. Commun. Netw., vol. 11, no. 5, pp. 151-163, May 2019.

[35] K. Kiasaleh, "Hybrid ARQ for FSO communications through turbulent atmosphere," IEEE Commun. Lett., vol. 14, no. 9, pp. 866-868, Aug. 2010.

[36] S. M. Aghajanzadeh and M. Uysal, "Information theoretic analysis of hybrid-ARQ protocols in coherent free-space optical systems," IEEE Trans. Commun., vol. 60, no. 5, pp. 1432-1442, Mar. 2012.

[37] M. A. Khalighi and M. Uysal, "Survey on free space optical communication: A communication theory perspective," IEEE Commun. Surveys Tuts., vol. 16, no. 4, pp. 2231-2258, Fourth quarter 2014.

[38] H. Kaushal and G. Kaddoum, "Optical communication in space: Challenges and mitigation techniques," IEEE Commun. Surveys Tuts., vol. 19, no. 1, pp. 57-96, First quarter 2017.

[39] I. K. Son and S. Mao, "A survey of free space optical networks," Digit. Commun. Netw., vol. 3, no. 2, pp. 67-77, May 2017.

[40] A. Vavoulas, H. G. Sandalidis, N. D. Chatzidiamantis, Z. Xu, and G. K. Karagiannidis, "A survey on ultraviolet C-band (UV-C) communications," IEEE Commun. Surveys Tuts., vol. 21, no. 3, pp. 2111-2133, Third quarter 2019.

[41] A. Trichili, K. Park, M. Zghal, B. S. Ooi, and M. Alouini, "Communicating using spatial mode multiplexing: Potentials, challenges, and perspectives," IEEE Commun. Surveys Tuts., vol. 21, no. 4, pp. 31753203, Fourth quarter 2019.

[42] A. B. Raj and A. K. Majumder, "Historical perspective of free space optical communications: from the early dates to today's developments," IET Commun., vol. 13, no. 16, pp. 2405-2419, Oct. 2019.

[43] R. Chen, H. Zhou, M. Moretti, X. Wang, and J. Li, "Orbital angular momentum waves: Generation, detection, and emerging applications," IEEE Commun. Surveys Tuts., vol. 22, no. 2, pp. 840-868, Second quarter 2020.

[44] M. Z. Chowdhury, M. K. Hasan, M. Shahjalal, M. T. Hossan, and Y. M. Jang, "Optical wireless hybrid networks: Trends, opportunities, challenges, and research directions," IEEE Commun. Surveys Tuts., vol. 22, no. 2, pp. 930-966, Second quarter 2020.

[45] W. Liu, J. Ding, J. Zheng, X. Chen, and C. L. I, "Relay-assisted technology in optical wireless communications: A survey," IEEE Access, vol. 8, pp. 194384-194409, Nov. 2020.

[46] A. Trichili, M. A. Cox, B. S. Ooi, and M. S. Alouini, "Roadmap to free space optics," J. Opt. Soc. Am. B, vol. 37, no. 11, pp. 184-201, Nov. 2020.

[47] Shu Lin, D. J. Costello, and M. J. Miller, "Automatic-repeat-request error-control schemes," IEEE Commun. Mag., vol. 22, no. 12, pp. 5-17, Dec. 1984.

[48] T. de Cola, E. Paolini, G. Liva, and G. P. Calzolari, "Reliability options for data communications in the future deep-space missions," Proc. IEEE, vol. 99, no. 11, pp. 2056-2074, Aug. 2011.

[49] R. Zhang and L. Hanzo, "A unified treatment of superposition coding aided communications: Theory and practice," IEEE Commun. Surveys Tuts., vol. 13, no. 3, pp. 503-520, Third quarter 2011.

[50] H. Chen, R. G. Maunder, and L. Hanzo, "A survey and tutorial on lowcomplexity turbo coding techniques and a holistic hybrid ARQ design example," IEEE Commun. Surveys Tuts., vol. 15, no. 4, pp. 1546-1566, First quarter 2013.

[51] H. A. Ngo and L. Hanzo, "Hybrid automatic-repeat-request systems for cooperative wireless communications," IEEE Commun. Surveys Tuts., vol. 16, no. 1, pp. 25-45, Fourth quarter 2014.

[52] H. Mukhtar, A. Al-Dweik, and A. Shami, "Turbo product codes: Applications, challenges, and future directions," IEEE Commun. Surveys Tuts., vol. 18, no. 4, pp. 3052-3069, Fourth quarter 2016.

[53] S. Jiang, "On reliable data transfer in underwater acoustic networks: A survey from networking perspective," IEEE Commun. Surveys Tuts., vol. 20, no. 2, pp. 1036-1055, Second quarter 2018.
[54] B. Makki, K. Chitti, A. Behravan, and M. S. Alouini, "A survey of NOMA: Current status and open research challenges," IEEE Open J. Commun. Soc., vol. 1, pp. 179-189, Jan. 2020.

[55] A. Ahmed, A. Al-Dweik, Y. Iraqi, H. Mukhtar, M. Naeem, and E. Hossain, "Hybrid automatic repeat request (HARQ) in wireless communications systems and standards: A contemporary survey," IEEE Commun. Surveys Tuts., pp. 1-1, Jul. 2021.

[56] Y. Qin and L. Yang, "Steady-state throughput analysis of network coding nodes employing stop-and-wait automatic repeat request," IEEE/ACM Trans. Netw., vol. 20, no. 5, pp. 1402-1411, Dec. 2012.

[57] P. Larsson, L. K. Rasmussen, and M. Skoglund, "Throughput analysis of arq schemes in gaussian block fading channels," IEEE Trans. Commun., vol. 62, no. 7, pp. 2569-2588, May 2014.

[58] F. Cao, Y. Song, and Y. Yang, "Arq assisted short-packet communications for noma networks over nakagami-m fading channels," IEEE Access, vol. 8, pp. 158263-158272, Aug. 2020.

[59] J. F. Kurose and K. W. Ross, Computer Networking: A Top-Down Approach. London, UK: Pearson, 6th ed., 2012.

[60] A. L. Garcia and I. Widjaja, Communication Networks: Fundamental Concepts and Key Architectures. USA: McGraw-Hill, Inc., 2nd ed., 2003.

[61] S. D. Milner, S. Trisno, C. C. Davis, B. Epple, and H. Henniger, "A cross-layer approach to mitigate fading on bidirectional free space optical communication links," in Proc. IEEE Military Commun. Conf., pp. 1-6, 2008 .

[62] G. Prakash, A. Nayak, M. Kulkarni, and S. Acharya, "On the improved performance of luby transform codes over selective repeat ARQ in turbulent free space optical links," in Proc. IEEE Int. Conf. Comput. Sci. Eng, pp. 1195-1200, 2013.

[63] V. V. Mai and A. T. Pham, "Performance analysis of cooperativeARQ schemes in free-space optical communications," IEICE Trans. Commun., vol. E94.B, no. 8, pp. 1614-1622, Jan. 2011.

[64] S. Parthasarathy, D. Giggenbach, and A. Kirstaedter, "Channel modelling for free-space optical inter-HAP links using adaptive ARQ transmission," in Proc. SPIE Unmanned/Unattended Sensors and Sensor Netw. $X$, pp. 170-180, 2014.

[65] S. Parthasarathy, A. Kirstaedter, and D. Giggenbach, "Simulative performance analysis of ARQ schemes for free-space optical inter-HAP channel model," in Proc. IEEE Photonic Netw., pp. 1-5, 2015.

[66] V. V. Mai and A. T. Pham, "Adaptive multi-rate designs for hybrid FSO/RF systems over fading channels," in Proc. IEEE Global Commun. Conf., pp. 469-474, 2014.

[67] V. V. Mai and A. T. Pham, "Adaptive multi-rate designs and analysis for hybrid FSO/RF systems over fading channels," IEICE Trans. Commun., vol. E98.B, no. 8, pp. 1660-1671, Aug. 2015.

[68] V. V. Mai, T. C. Thang, and A. T. Pham, "Cross-layer design and analysis for FSO links using automatic repeat request and adaptive modulation/coding schemes," in Proc. Int Symp. Commun. Syst. Netw. Digit. Signal Process., pp. 1176-1180, 2014.

[69] L. Clare and G. Miles, "Deep space optical link ARQ performance analysis," in Proc. IEEE Aerosp. Conf., pp. 1-11, 2016.

[70] C. M. Schieler, B. S. Robinson, and D. M. Boroson, "Data delivery performance of space-to-ground optical communication systems employing rate-constrained feedback protocols," in Proc. SPIE Free-Space Las. Comm. Atmos. Prop., pp. 325-332, 2017.

[71] B. Makki, T. Svensson, T. Eriksson, and M.-S. Alouini, "Performance analysis of ARQ-based RF-FSO links," IEEE Commun. Lett., vol. 21, no. 6, pp. 1253-1256, Feb. 2017.

[72] H. D. Le, V. V. Mai, C. T. Nguyen, T. C. Thang, and A. T. Pham, "Modeling and throughput analysis of FSO systems using GBN-ARQ and AR transmission over atmospheric turbulence channels," in Proc. Int. Symp. Commun. Syst. Netw. Digit. Signal Process., pp. 1-6, 2018.

[73] H. D. Le, V. V. Mai, C. T. Nguyen, and A. T. Pham, "Sliding window protocols with rate adaptation for FSO burst transmission over turbulence channels," in Proc. Int. Conf. Inform. Commun. Technol. Convergence, pp. 821-826, 2018.

[74] C. M. Schieler, A. S. Garg, B. C. Bilyeu, J. P. Wang, and B. S Robinson, "Demonstration of reliable high-rate optical communication over an atmospheric link using ARQ," in Proc. IEEE Int. Conf. Space Optical Syst. Appl., pp. 1-6, 2019.

[75] M. R. Aghaei, A. M. A. Hemmatyar, A. Chamanmotlagh, and M. Fouladian, "Analysis of adaptive multi-rate FSO/RF hybrid systems using malaga-M distribution model in turbulent channels," J. Modern Optics, vol. 67, no. 13, pp. 1159-1169, Sept. 2020.

[76] L. C. Andrews, R. L. Phillips, C. Y. Hopen, and M. A. Al-Habash, "Theory of optical scintillation," J. Opt. Soc. Am. A, vol. 16, no. 6, pp. 1417-1429, Jun. 1999. 
[77] L. C. Andrews, R. L. Phillips, and C. Y. Hopen, Laser Beam Scintillation with Applications. Bellingham, WA: SPIE Press, 1st ed., 2001.

[78] M. A. Al-Habash, L. C. Andrews, and R. L. Phillips, "Mathematical model for the irradiance probability density function of a laser beam propagating through turbulent media," Opt. Eng., vol. 40, no. 8, pp. 1554-1562, Aug. 2001.

[79] A. K. Majumdar, "Free-space laser communication performance in the atmospheric channel," J. Opt. Fiber Commun. Rep., vol. 2, no. 4, pp. 345-396, Nov. 2005.

[80] A. Jurado-Navas, A. Garcia-Zambrana, and A. Puerta-Notario, "Efficient lognormal channel model for turbulent FSO communications," IET Electron. Lett., vol. 43, no. 3, pp. 178-179, Feb. 2007.

[81] A. Jurado-Navas, J. M. Garrido-Balsells, M. Castillo-Vazquez, and A. Puerta-Notario, "An efficient rate-adaptive transmission technique using shortened pulses for atmospheric optical communications," OSA Opt. Express, vol. 18, no. 16, pp. 17346-17363, Aug. 2010.

[82] C. Abou-Rjeily, "Achievable diversity orders of decode-and-forward cooperative protocols over gamma-gamma fading FSO links," IEEE Trans. Commun., vol. 61, no. 9, pp. 3919-3930, Sep. 2013.

[83] C. Abou-Rjeily and W. Fawaz, "Buffer-aided relaying protocols for cooperative FSO communications," IEEE Trans. Wireless Commun., vol. 16 , no. 12 , pp. 8205-8219, Dec. 2017.

[84] A. Bhowal and R. S. Kshetrimayum, "Transmit laser selection for two hop decode and forward FSO communication with pointing errors," IEEE Commun. Lett., vol. 23, no. 12, pp. 2301-2305, Dec. 2019.

[85] C. Abou-Rjeily and A. Slim, "Cooperative diversity for free-space optical communications: Transceiver design and performance analysis," IEEE Trans. Commun., vol. 59, no. 3, pp. 658-663, Mar. 2011.

[86] L. B. Stotts, L. C. Andrews, P. C. Cherry, J. J. Foshee, P. J. Kolodzy, W. K. McIntire, M. Northcott, R. L. Phillips, H. A. Pike, B. Stadler, and D. W. Young, "Hybrid optical RF airborne communications," Proc. IEEE, vol. 97, no. 6, pp. 1109-1127, Jun. 2009.

[87] A. Shlomi, B. John, K. George, S. Robert, and U. Murat, Advanced Optical Wireless Communication Systems. Cambridge, UK: Cambridge University Press, 2nd ed., 2012.

[88] H. D. Le, T. V. Nguyen, and A. T. Pham, "Cloud attenuation statistical model for satellite-based FSO communications," IEEE Antennas Wireless Propag. Lett., vol. 20, no. 5, pp. 643-647, May 2021.

[89] S. Rangan, T. S. Rappaport, and E. Erkip, "Millimeter-wave cellular wireless networks: Potentials and challenges," Proc. IEEE, vol. 102, no. 3, pp. 366-385, Mar. 2014.

[90] A. Mostafa and S. Hranilovic, "Channel measurement and markov modeling of an urban free-space optical link," IEEE/OSA J. Opt. Commun. Netw., vol. 4, no. 10, pp. 836-846, Oct. 2012.

[91] A. U. Chaudhry and H. Yanikomeroglu, "Free space optics for nextgeneration satellite networks," IEEE Consum. Electron. Mag., pp. 1-1, 2020.

[92] M. Toyoshima, "Recent trends in space laser communications for small satellites and constellations," J. Lightwave Technol., vol. 39, no. 3, pp. 693-699, Feb. 2021.

[93] H. Hamid, Near-Earth Laser Communications. Boca Raton, FL, USA: CRC Press, 2nd ed., 2020.

[94] B. S. Robinson, D. M. Boroson, C. M. Schieler, F. I. Khatri, O. Guldner, S. Constantine, T. Shih, J. W. Burnside, B. C. Bilyeu, F. Hakimi, A. Garg, G. Allen, E. Clements, and D. M. Cornwell, "Terabyte infrared delivery (TBIRD): A demonstration of large-volume direct-to-earth data transfer from low-earth orbit," in Proc. SPIE Free-Space Laser Commun. Atmospheric Propag. XXX, pp. 253-258, 2018.

[95] G. Xu and M. Zeng, "Solar scintillation effect for optical waves propagating through gamma-gamma coronal turbulence channels," IEEE Photon. J., vol. 11, no. 4, pp. 1-15, Aug. 2019.

[96] G. Xu and Q. Zhang, "Mixed RF/FSO deep space communication system under solar scintillation effect," IEEE Trans. Aerosp. Electron. Syst., pp. 1-1, 2021.

[97] G. Xu and Z. Song, "Effects of solar scintillation on deep space communications: Challenges and prediction techniques," IEEE Wireless Commun., vol. 26, no. 2, pp. 10-16, Apr. 2019.

[98] T. De Cola and M. Marchese, "Reliable data delivery over deep space networks: Benefits of long erasure codes over ARQ strategies," IEEE Wireless Commun., vol. 17, no. 2, pp. 57-65, Apr. 2010.

[99] Q. Yu, S. C. Burleigh, R. Wang, and K. Zhao, "Performance modeling of licklider transmission protocol (LTP) in deep-space communication," IEEE Trans. Aerosp. Electron. Syst., vol. 51, no. 3, pp. 1609-1620, Jul. 2015.

[100] S. Burleigh, A. Hooke, L. Torgerson, K. Fall, V. Cerf, B. Durst, K. Scott, and H. Weiss, "Delay-tolerant networking: an approach to interplanetary internet," IEEE Commun. Mag., vol. 41, no. 6, pp. 128 136, Jun. 2003.

[101] S. Sharma, A. S. Madhukumar, and R. Swaminathan, "Switchingbased cooperative decode-and-forward relaying for hybrid FSO/RF networks," IEEE/OSA J. Opt. Commun. Netw., vol. 11, no. 6, pp. 267281, Jun. 2019.

[102] M. Usman, H.-C. Yang, and M.-S. Alouini, "Practical switchingbased hybrid $\mathrm{FSO} / \mathrm{RF}$ transmission and its performance analysis," IEEE Photon. J., vol. 6, no. 5, pp. 1-13, 2014.

[103] J. M. Wozencraft and M. Horstein, "Digitalised communication over two-way channels," in The Fourth London Symposium of Information Theory, 1960.

[104] J. M. Wozencraft and M. Horstein, "Coding for two-way channels," in tech. rep. Research Laboratory of Electronics M.I.T, 1961.

[105] W. Lee, O. Simeone, J. Kang, S. Rangan, and P. Popovski, "Harq buffer management: An information-theoretic view," IEEE Trans. Commun. vol. 63, no. 11, pp. 4539-4550, Aug. 2015.

[106] E. Y. Rocher and R. L. Pickholtz, "An analysis of the effectiveness of hybrid transmission schemes," IBM J. Research Develop., vol. 14 no. 4, pp. 426-433, Jul. 1970.

[107] A. Sastry, "Performance of hybrid error control schemes of satellite channels," IEEE Trans. Commun., vol. 23, no. 7, pp. 689-694, Jul. 1975.

[108] A. Sastry and L. Kanal, "Hybrid error control using retransmission and generalized burst-trapping codes," IEEE Trans. Commun., vol. 24, no. 4, pp. 385-393, Apr. 1976.

[109] C. Fujiwara, M. Kasahara, K. Yamashita, and T. Namekawa, "Evaluations of error control techniques in both independent-error and dependent-error channels," IEEE Trans. Commun., vol. 26, no. 6, pp. 785-794, Jun. 1978.

[110] A. Drukarev and D. J. Costello, "A comparison of block and convolutional codes in ARQ error control schemes," IEEE Trans. Commun., vol. 30, no. 11, pp. 2449-2455, Nov. 1982.

[111] R. Joda and M. Zorzi, "Access policy design for cognitive secondary users under a primary type-I HARQ process," IEEE Trans. Commun., vol. 63, no. 11, pp. 4037-4049, Sep. 2015.

[112] M. Maaz, P. Mary, and M. Helard, "Energy minimization in HARQ-I relay-assisted networks with delay-limited users," IEEE Trans. Veh. Technol., vol. 66, no. 8, pp. 6887-6898, Jan. 2017.

[113] X. Leturc, P. Ciblat, and C. J. Le Martret, "Energy efficient resource allocation for type-I HARQ under the rician channel," IEEE Trans. Wireless Commun., vol. 18, no. 7, pp. 3739-3751, May 2019.

[114] S. Lin and P. Yu, "A hybrid ARQ scheme with parity retransmission for error control of satellite channels," IEEE Trans. Commun., vol. 30, no. 7, pp. 1701-1719, 1982.

[115] D. Chase, "Code combining - a maximum-likelihood decoding approach for combining an arbitrary number of noisy packets," IEEE Trans. Commun., vol. 33, no. 5, pp. 385-393, May 1985.

[116] H. Long, W. Xiang, S. Shen, Y. Zhang, K. Zheng, and W. Wang, "Analysis of conditional error rate and combining schemes in HARQ," IEEE Trans. Signal Process., vol. 60, no. 5, pp. 2677-2682, Jan. 2012.

[117] E. Dahlman, S. Parkvall, and J. Skol, 4G: LTE/LTE-Advanced for Mobile Broadband. NewYork, NY, USA: Academic, 1st ed., 2013.

[118] J.-F. Cheng, "Coding performance of hybrid ARQ schemes," IEEE Trans. Commun., vol. 54, no. 6, pp. 1017-1029, Jun. 2006.

[119] C. Kose and T. R. Halford, "Incremental redundancy hybrid ARQ protocol design for FSO links," in Proc. IEEE Military Commun. Conf., pp. $1-7,2009$

[120] A. Abdulhussein, A. Oka, T. T. Nguyen, and L. Lampe, "Rateless coding for hybrid free-space optical and radio-frequency communication," IEEE Trans. Wireless Commun., vol. 9, no. 3, pp. 907-913, Mar. 2010.

[121] J. Perez-Ramirez and D. K. Borah, "Design and analysis of bit selections in HARQ algorithm for hybrid FSO/RF channels," in Proc. IEEE Veh, Tech. Conf., pp. 1-5, 2013.

[122] E. Zedini, A. Chelli, and M. Alouini, "On the performance analysis of hybrid ARQ with incremental redundancy and with code combining over free-space optical channels with pointing errors," IEEE Photon. J., vol. 6, no. 4, pp. 1-18, Jul. 2014.

[123] B. Makki, T. Svensson, T. Eriksson, and M. S. Alouini, "On the performance of HARQ-based RF-FSO links," in Proc. IEEE Global Commun. Conf., pp. 1-7, 2015.

[124] B. Makki, T. Svensson, T. Eriksson, and M. S. Alouini, "On the performance of RF-FSO links with and without hybrid ARQ," IEEE Trans. Wireless Commun., vol. 15, no. 7, pp. 4928-4943, Apr. 2016.

[125] K. Kiasaleh, "Hybrid ARQ communications for severely degraded hermite-gaussian FSO link," in Proc. IEEE Int. Conf. Space Opt., pp. 17, 2016. 
[126] S. Parthasarathy, A. Kirstaedter, and D. Giggenbach, "Performance analysis of adaptive hybrid ARQ for inter-hap free-space optical fading channel with delayed channel state information," in Proc. IEEE Photonic Netw., pp. 1-7, 2016.

[127] S. Parthasarathy, A. Kirstaedter, and D. Giggenbach, "Adaptive HARQ with channel state information in inter-HAP FSO links," in Proc. IEEE Photonic Netw., pp. 1-6, 2017.

[128] B. Makki, T. Svensson, M. Brandt-Pearce, and M. S. Alouini, "On the performance of millimeter wave-based RF-FSO multi-hop and mesh networks," IEEE Trans. Wireless Commun., vol. 16, no. 12, pp. 77467759, Sep. 2017.

[129] A. Touati, M. O. Hasna, and F. Touati, "HARQ performance over FSO channels with atmospheric fading and pointing errors," in Proc. IEEE Int. Wireless Comm. Mob. Comput. Conf., pp. 158-163, 2018.

[130] J. Xiang, X. Lu, and W. Qiao, "Research on anti fading transmission technology of improved HARQ scheme in space optical communication," in Proc. IEEE Int. Conf. Intell. Comput. Autom. and Syst., pp. 897-901, 2019.

[131] S. S. Hosseini, J. Abouei, B. Champagne, and X. Chang, "A novel cooperative HARQ protocol for free-space optical broadcasting systems," J. Lightwave Technol., vol. 38, no. 7, pp. 1789-1799, Feb. 2020.

[132] H. D. Le, V. V. Mai, C. T. Nguyen, and A. T. Pham, "Throughput analysis of incremental redundancy hybrid ARQ for FSO-based satellite systems," in Proc. IEEE Veh, Tech. Conf., pp. 1-5, 2019.

[133] H. D. Le and A. T. Pham, "On the design of FSO-based satellite systems using incremental redundancy hybrid ARQ protocols with rate adaptation," IEEE Trans. Veh. Technol., May 2021 (Accepted).

[134] H. D. Nguyen, H. D. Le, C. T. Nguyen, and A. T. Pham, "Throughput and delay performance of cooperative HARQ in satellite-HAP-vehicle FSO systems," in Proc. IEEE Veh, Tech. Conf., pp. 1-6, 2021.

[135] J. Park, E. Lee, C.-B. Chae, and G. Yoon, "Impact of pointing errors on the performance of coherent free-space optical systems," IEEE Photon. Tech. Lett., vol. 28, no. 2, pp. 181-184, Jan. 2016.

[136] S. Bae, J. Park, S. Kim, and G. Yoon, "Performance analysis of coherent FSO-OFDM systems with frequency offset," IEEE Commun. Lett., vol. 20, no. 11, pp. 2189-2192, Nov. 2016.

[137] L. Li, T. Geng, Y. Wang, X. Li, J. Wu, Y. Li, S. Ma, S. Gao, and Z. Wu, "Free-space optical communication using coherent detection and double adaptive detection thresholds," IEEE Photon. J., vol. 11, no. 1, pp. 1-17, Feb. 2019.

[138] C. Jia, P. Wang, Y. Li, C. Huang, H. Fu, and W. Pang, "ABERs of LDPC-coded multi-hop FSO over double gg fading channels with pointing error and path loss," IEEE Photonics Technol. Lett., vol. 30, no. 15, pp. 1357-1360, Aug. 2018.

[139] D. Agarwal and A. Bansal, "Unified error performance of a multihop DF-FSO network with aperture averaging," IEEE/OSA J. Opt. Commun. Netw., vol. 11, no. 3, pp. 95-106, Mar. 2019.

[140] E. Saleh Altubaishi and K. Alhamawi, "Capacity analysis of hybrid AF multi-hop FSO/RF system under pointing errors and weather effects," IEEE Photonics Technol. Lett., vol. 31, no. 15, pp. 1304-1307, 2019.

[141] I. A. Hemadeh, K. Satyanarayana, M. El-Hajjar, and L. Hanzo, "Millimeter-wave communications: Physical channel models, design considerations, antenna constructions, and link-budget," IEEE COmmun. Surveys Tuts., vol. 20, no. 2, pp. 870-913, Second quarter 2018.

[142] E. Zedini, H. Soury, and M.-S. Alouini, "On the performance analysis of dual-hop mixed FSO/RF systems," IEEE Trans. Wireless Commun., vol. 15, no. 5, pp. 3679-3689, May 2016.

[143] H. Zhou, D. G. M. Mitchell, N. Goertz, and D. J. Costello, "Robust rate-compatible punctured LDPC convolutional codes," IEEE Trans. Commun., vol. 61, no. 11, pp. 4428-4439, Nov. 2013.

[144] S.-N. Hong and M.-O. Jeong, "An efficient construction of ratecompatible punctured polar (RCPP) codes using hierarchical puncturing," IEEE Trans. Commun., vol. 66, no. 11, pp. 5041-5052, Nov. 2018.

[145] M. Luby, "LT codes," in Proc. IEEE Symp. on Foundations of Comp. Sc., pp. 271-280, 2002.

[146] A. Abdulhussein, A. Oka, T. T. Nguyen, and L. Lampe, "Rateless coding for hybrid free-space optical and radio-frequency communication," IEEE Trans. Wireless Commun., vol. 9, no. 3, pp. 907-913, Mar. 2010.

[147] A. Shokrollahi, "Raptor codes," IEEE Trans. Inf. Theory, vol. 52, no. 6, pp. 2551-2567, Jun. 2006.

[148] Y. Yamashita, E. Okamoto, Y. Iwanami, Y. Shoji, M. Toyoshima, and Y. Takayama, "An efficient LDGM coding scheme for optical satelliteto-ground link based on a new channel model," in Proc. IEEE Global Commun. Conf., pp. 1-6, 2010.
[149] G. Karabulut Kurt, M. G. Khoshkholgh, S. Alfattani, A. Ibrahim, T. S. J. Darwish, M. S. Alam, H. Yanikomeroglu, and A. Yongacoglu, "A vision and framework for the high altitude platform station (HAPS) networks of the future," IEEE Commun. Surveys Tuts., vol. 23, no. 2, pp. 729-779, Second quarter 2021.

[150] S. Sesia, G. Caire, and G. Vivier, "Incremental redundancy hybrid ARQ schemes based on low-density parity-check codes," IEEE Trans. Commun., vol. 52, no. 8, pp. 1311-1321, Aug. 2004.

[151] P. Rost and A. Prasad, "Opportunistic hybrid ARQ - enabler of centralized-RAN over nonideal backhaul," IEEE Wireless Commun. Lett., vol. 3, no. 5, pp. 481-484, Oct. 2014.

[152] B. Makki, T. Svensson, G. Caire, and M. Zorzi, "Fast HARQ over finite blocklength codes: A technique for low-latency reliable communication," IEEE Trans. Wireless Commun., vol. 18, no. 1, pp. 194-209, Jan. 2019.

[153] G. Cerar, H. Yetgin, M. Mohorcic, and C. Fortuna, "Machine learning for wireless link quality estimation: A survey," IEEE Commun. Surveys Tuts., vol. 23, no. 2, pp. 696-728, Second Quarter 2021.

[154] N. Strodthoff, B. Goktepe, T. Schierl, C. Hellge, and W. Samek, "Enhanced machine learning techniques for early HARQ feedback prediction in 5G," IEEE J. Sel. Areas Commun., vol. 37, no. 11, pp. 2573-2587, Nov. 2019.

[155] F. Foukalas, V. Gazis, and N. Alonistioti, "Cross-layer design proposals for wireless mobile networks: A survey and taxonomy," IEEE Commun. Surveys Tuts., vol. 10, no. 1, pp. 70-85, First Quarter 2008.

[156] X. Lin, N. Shroff, and R. Srikant, "A tutorial on cross-layer optimization in wireless networks," IEEE J. Sel. Areas Commun., vol. 24, no. 8, pp. 1452-1463, Aug. 2006.

[157] Q. Liu, S. Zhou, and G. Giannakis, "Cross-layer combining of adaptive modulation and coding with truncated ARQ over wireless links," IEEE Trans. Wireless Commun., vol. 3, no. 5, pp. 1746-1755, Sep. 2004.

[158] B. Fu, Y. Xiao, H. Deng, and H. Zeng, "A survey of cross-layer designs in wireless networks," IEEE Commun. Surveys Tuts., vol. 16, no. 1 , pp. 110-126, First Quarter 2014.

[159] A. Awang, K. Husain, N. Kamel, and S. Aissa, "Routing in vehicular ad-hoc networks: A survey on single- and cross-layer design techniques, and perspectives," IEEE Access, vol. 5, pp. 9497-9517, Apr. 2017.

[160] Q. Medhat Salih, M. A. Rahman, F. Al-Turjman, and Z. R. M. Azmi, "Smart routing management framework exploiting dynamic data resources of cross-layer design and machine learning approaches for mobile cognitive radio networks: A survey," IEEE Access, vol. 8, pp. 67835-67867, Apr. 2020.

[161] V. Srivastava and M. Motani, "Cross-layer design: a survey and the road ahead," IEEE Commun. Mag., vol. 43, no. 12, pp. 112-119, Dec. 2005.

[162] C. T. Nguyen, H. D. Le, and V. V. Mai, "A cross-layer analysis of $\mathrm{TCP} /$ link adaptation technologies over free-space optical links with markov error model," Springer Photon. Netw. Commun., vol. 36, no. 1 , pp. 279-288, Sep. 2018.

[163] L. Le, E. Hossain, and T. Le-Ngoc, "Interaction between radio link level truncated ARQ, and TCP in multi-rate wireless networks: a cross-layer performance analysis," IET Commun., vol. 1, no. 5, pp. 821-830, Oct. 2007.

[164] R. Edirisinghe and A. Zaslavsky, "Cross-layer contextual interactions in wireless networks," IEEE Commun. Surveys Tuts., vol. 16, no. 2, pp. 1114-1134, Second Quarter 2014.

[165] A. Argyriou, "Cross-layer error control for multimedia streaming in wireless/wireline packet networks," IEEE Trans. Multimedia, vol. 10, no. 6, pp. 1121-1127, Oct. 2008.

[166] V. Kawadia and P. Kumar, "A cautionary perspective on cross-layer design," IEEE Wireless Commun., vol. 12, no. 1, pp. 3-11, Feb. 2005.

[167] V. V. Mai, T. C. Thang, and A. T. Pham, "Performance of TCP over free-space optical atmospheric turbulence channels," IEEE/OSA J. Opt. Commun. Netw., vol. 5, no. 11, pp. 1168-1177, Nov. 2013.

[168] C. V. Le, P. V. Trinh, T. C. Thang, and A. T. Pham, "PHY error models for NS-2 simulation of FSO packet networks over turbulence channels," in Proc. Int Symp. Commun. Syst. Netw. Digit. Signal Process., pp. 1-6, 2018.

[169] H. D. Le, T. V. Pham, P. V. Trinh, D. R. Kolev, A. C. Casado, and A. T. Pham, "Performance of TCP in FSO-based LEO satellite systems," in Proc. IEEE Int. Conf. on Space Opt. Sys. App., pp. 1-6, 2019.

[170] H. D. Le and A. T. Pham, "TCP over satellite-to-unmanned aerial/ground vehicles laser links: Hybla or cubic?," in IEEE Region 10 Conf., pp. 720-725, 2020. 
[171] T. K. Nguyen, C. T. Nguyen, H. D. Le, and A. T. Pham, "TCP performance over satellite-based hybrid FSO/RF vehicular networks: Modeling and analysis," IEEE Access, Jul. 2021 (Accepted).

[172] F. Moll, "Experimental analysis of channel coherence time and fading behavior in the LEO-ground link," in Proc. IEEE Int. Conf. on Space Opt. Sys. App., pp. 1-7, 2014.

[173] D. J. Geisler, C. M. Schieler, T. M. Yarnall, M. L. Stevens, B. S. Robinson, and S. A. Hamilton, "Demonstration of a variable data-rate free-space optical communication architecture using efficient coherent techniques," SPIE Optical Eng., vol. 55, no. 11, pp. 1-12, Aug. 2016.

[174] I. B. Djordjevic, "Adaptive modulation and coding for free-space optical channels," IEEE/OSA J. Opt. Commun. Netw., vol. 2, no. 5, pp. 221-229, May 2010.

[175] A. G. Zambrana, C. C. Vazquez, and B. C. Vazquez, "Rate-adaptive FSO links over atmospheric turbulence channels by jointly using repetition coding and silence periods," Opt. Express, vol. 18, no. 24, pp. 25422-25440, Nov. 2010.

[176] Z. Hassan, J. Hossain, J. Cheng, and V. C. Leung, "Delay-QoS-aware adaptive modulation and power allocation for dual-channel coherent OWC," IEEE/OSA J. Opt. Commun. Netw., vol. 10, no. 3, pp. 138151, Mar. 2018.

[177] A. Elzanaty and M.-S. Alouini, "Adaptive coded modulation for im/dd free-space optical backhauling: A probabilistic shaping approach," IEEE Trans. Comm., vol. 68, no. 10, pp. 6388-6402, Oct. 2020.

[178] J. Yun and M. Kavehrad, "Markov error structure for throughput analysis of adaptive modulation systems combined with ARQ over correlated fading channels," IEEE Trans. Veh. Technol., vol. 54, no. 1, pp. 235-245, Jan. 2005.

[179] M. Polese, F. Chiariotti, E. Bonetto, F. Rigotto, A. Zanella, and M. Zorzi, "A survey on recent advances in transport layer protocols," IEEE Commun. Surveys Tuts., vol. 21, no. 4, pp. 3584-3608, Fourth Quarter 2019.

[180] B. Sardar and D. Saha, "A survey of TCP enhancements for last-hop wireless networks," IEEE Commun. Surveys Tuts., vol. 8, no. 3, pp. 20 34, Third Quarter 2006.

[181] K.-C. Leung and V. O. Li, "Transmission control protocol (TCP) in wireless networks: issues, approaches, and challenges," IEEE Commun. Surveys Tuts., vol. 8, no. 4, pp. 64-79, Fourth Quarter 2006.

[182] S. R. Pokhrel, M. Panda, H. L. Vu, and M. Mandjes, "TCP performance over Wi-Fi: Joint impact of buffer and channel losses," IEEE Trans. Mobile Comput., vol. 15, no. 5, pp. 1279-1291, May 2016.

[183] X. Li and J. Wang, "Elastically reliable video transport protocol over lossy satellite links," IEEE J. Sel. Areas Commun., vol. 36, no. 5, pp. 1097-1108, May 2018.

[184] J. Liu, Z. Han, and W. Li, "Performance analysis of TCP new reno over satellite DVB-RCS2 random access links," IEEE Trans. Wireless Commun., vol. 19, no. 1, pp. 435-446, Oct. 2020.

[185] Y. Hasegawa, "A transmission control protocol for free-space optical communications," in Proc. IEEE Global Commun. Conf., pp. 1-7, 2017.

[186] Y. Hasegawa, T. Ito, Y. Ono, and M. Arikawa, "A throughput model of TCP-FSO/ADFR for free-space optical satellite communications," in Proc. IEEE Global Commun. Conf., pp. 1-6, 2019.

[187] A. Toledo and X. Wang, "TCP performance over wireless MIMO channels with ARQ and packet combining," IEEE Trans. Mobile Comput., vol. 5, no. 3, pp. 208-223, Mar. 2006.

[188] A. Toledo, X. Wang, and B. Lu, "A cross-layer TCP modelling framework for MIMO wireless systems," IEEE Trans. Wireless Commun., vol. 5, no. 4, pp. 920-929, Apr. 2006.

[189] F. Vacirca, A. De Vebductis, and A. Baiocchi, "Optimal design of hybrid FEC/ARQ schemes for TCP over wireless links with rayleigh fading," IEEE Trans. Mobile Comput., vol. 5, no. 4, pp. 289-302, Apr. 2006.

[190] Q. Huang, S. Chan, L. Ping, and M. Zukerman, "Improving wireless TCP throughput by a novel TCM-based hybrid ARQ," IEEE Trans. Wireless Commun., vol. 6, no. 7, pp. 2476-2485, Jul. 2007.

[191] R. Zhang and L. Hanzo, "Superposition-coding-aided multiplexed hybrid ARQ scheme for improved end-to-end transmission efficiency," IEEE Trans. Veh. Technol., vol. 58, no. 8, pp. 4681-4686, Oct. 2009.

[192] H. D. Le, C. T. Nguyen, V. V. Mai, and A. T. Pham, "On the throughput performance of TCP cubic in millimeter-wave cellular networks," IEEE Access, vol. 7, pp. 178618-178630, Dec. 2019.

[193] E. Lee and V. Chan, "Performance of the transport layer protocol for diversity communication over the clear turbulent atmospheric optical channel," in Proc. IEEE Int. Conf. Commun., vol. 1, pp. 333-339, 2005.
[194] M. Zhang, M. Mezzavilla, R. Ford, S. Rangan, S. Panwar, E. Mellios, D. Kong, A. Nix, and M. Zorzi, "Transport layer performance in 5G mmwave cellular," in Proc. IEEE Conf. Comput. Commun. Workshops, pp. 730-735, 2016.

[195] Q. Zhang and S. Kassam, "Finite-state markov model for rayleigh fading channels," IEEE Trans. Commun., vol. 47, no. 11, pp. 16881692, Nov. 1999.

[196] G. Caire and D. Tuninetti, "The throughput of hybrid-ARQ protocols for the gaussian collision channel," IEEE Trans. Inf. Theory, vol. 47, no. 5, pp. 1971-1988, Jul. 2001.

[197] S. Shamai and A. Wyner, "Information-theoretic considerations for symmetric, cellular, multiple-access fading channels. i," IEEE Trans. Inf. Theory, vol. 43, no. 6, pp. 1877-1894, Nov. 1997.

[198] S. Dang, O. Amin, B. Shihada, and M. S. Alouini, "What should 6G be?," Nature Electron., vol. 3, no. 1, pp. 20-29, Jan. 2020.

[199] I. F. Akyildiz, A. Kak, and S. Nie, "6G and beyond: The future of wireless communications systems," IEEE Access, vol. 8, pp. 133995134030, Jul. 2020.

[200] L. Bariah, L. Mohjazi, S. Muhaidat, P. C. Sofotasios, G. K. Kurt, H. Yanikomeroglu, and O. A. Dobre, "A prospective look: Key enabling technologies, applications and open research topics in 6G networks," IEEE Access, vol. 8, pp. 174792-174820, Aug. 2020.

[201] W. Jiang, B. Han, M. A. Habibi, and H. D. Schotten, "The road towards 6G: A comprehensive survey," IEEE Open J. Commun. Soc., vol. 2, pp. 334-366, Feb. 2021

[202] H. Tataria, M. Shafi, A. F. Molisch, M. Dohler, H. Sjoland, and F. Tufvesson, "6G wireless systems: Vision, requirements, challenges, insights, and opportunities," Proc. IEEE, vol. 109, no. 7, pp. 11661199, Jul. 2021.

[203] G. J. Sutton, J. Zeng, R. P. Liu, W. Ni, D. N. Nguyen, B. A Jayawickrama, X. Huang, M. Abolhasan, Z. Zhang, E. Dutkiewicz, and $\mathrm{T}$. Lv, "Enabling technologies for ultra-reliable and low latency communications: From PHY and MAC layer perspectives," IEEE Commun. Surveys Tuts., vol. 21, no. 3, pp. 2488-2524, Third Quarter 2019.

[204] X. You and et. al., "Towards 6G wireless communication networks: vision, enabling technologies, and new paradigm shifts," Springer China Inform. Sci., vol. 64, no. 1, pp. 1869-1919, Jan. 2021.

[205] D. Feng, C. Jiang, G. Lim, L. J. Cimini, G. Feng, and G. Y. Li, "A survey of energy-efficient wireless communications," IEEE Commun. Surveys Tuts., vol. 15, no. 1, pp. 167-178, First Quarter 2013.

[206] P. Porambage, G. Gur, D. P. M. Osorio, M. Liyanage, A. Gurtov, and M. Ylianttila, "The roadmap to 6G security and privacy," IEEE Open J. Commun. Soc., vol. 2, pp. 1094-1122, May 2021.

[207] X. Guan, Y. Cai, and W. Yang, "On the reliability-security tradeoff and secrecy throughput in cooperative ARQ," IEEE Commun. Lett., vol. 18, no. 3, pp. 479-482, Mar. 2014.

[208] X. Tang, R. Liu, P. Spasojevic, and H. V. Poor, "On the throughput of secure hybrid-ARQ protocols for gaussian block-fading channels," IEEE Trans. Inf. Theory, vol. 55, no. 4, pp. 1575-1591, Apr. 2009.

[209] G. Qiu, M.-M. Zhao, M. Lei, and M.-j. Zhao, "Throughput maximization for polar coded IR-HARQ using deep reinforcement learning," in Proc. Int. Symp. Pers. Indoor Mobile Radio Commun., pp. 1-6, 2020.

[210] D. Liu, G. Zhu, Q. Zeng, J. Zhang, and K. Huang, "Wireless data acquisition for edge learning: Data-importance aware retransmission," IEEE Trans. Wireless Commun., vol. 20, no. 1, pp. 406-420, Jan. 2021.

[211] M. Grassl, L. Kong, Z. Wei, Z.-Q. Yin, and B. Zeng, "Quantum error-correcting codes for qudit amplitude damping," IEEE Trans. Inf. Theory, vol. 64, no. 6, pp. 4674-4685, Jun. 2018.

[212] Z. Babar, D. Chandra, H. V. Nguyen, P. Botsinis, D. Alanis, S. X. $\mathrm{Ng}$, and L. Hanzo, "Duality of quantum and classical error correction codes: Design principles and examples," IEEE Commun. Surveys Tuts., vol. 21, no. 1, pp. 970-1010, First Quarter 2019.

[213] Z. Babar, Z. B. Kaykac Egilmez, L. Xiang, D. Chandra, R. G. Maunder, S. X. Ng, and L. Hanzo, "Polar codes and their quantum-domain counterparts," IEEE Commun. Surveys Tuts., vol. 22, no. 1, pp. 123155, First Quarter 2020.

[214] N. Hosseinidehaj, Z. Babar, R. Malaney, S. X. Ng, and L. Hanzo, "Satellite-based continuous-variable quantum communications: Stateof-the-art and a predictive outlook," IEEE Commun. Surveys Tuts. vol. 21, no. 1, pp. 881-919, First Quarter 2019.

[215] A. Ashikhmin, "Fidelity of a quantum ARQ protocol," in Proc. IEEE Inform. Theory Workshop, pp. 42-46, 2006.

[216] J. Li, Z. Guo, and H. Ma, "Development of hybrid ARQ protocol for the quantum communication system on stabilizer codes," China Commun., vol. 18, no. 2, pp. 40-48, Feb. 2021. 